

\section{DISCLAIMER}

This report was prepared as an account of work sponsored by an agency of the United States Government. Neither the United States Government nor any agency Thereof, nor any of their employees, makes any warranty, express or implied, or assumes any legal liability or responsibility for the accuracy, completeness, or usefulness of any information, apparatus, product, or process disclosed, or represents that its use would not infringe privately owned rights. Reference herein to any specific commercial product, process, or service by trade name, trademark, manufacturer, or otherwise does not necessarily constitute or imply its endorsement, recommendation, or favoring by the United States Government or any agency thereof. The views and opinions of authors expressed herein do not necessarily state or reflect those of the United States Government or any agency thereof. 


\section{DISCLAIMER}

Portions of this document may be illegible in electronic image products. Images are produced from the best available original document. 
UNIVERSITY OF CALIFORNIA

Lawrence-Radiation Laboratory

Livermore, California

Contract No. W-7405-eng-48

END-LOSSES FROM MIRROR MACHINES

A. Physical Part

by

John.E. Roberts

'B. Computational Part

by

Marlene L. Carr

April 1960 

UCRL $5651-\mathrm{T}$

TABLE OF CONTENTS

Abstract .

\section{A - - PHYSICAL PART}

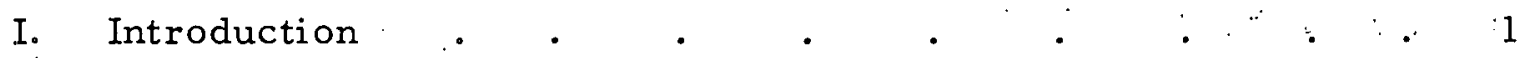

II. Basic Assumptions of the Rosenbluth-MacDonald-Judd Theory in This Treatment of the End-Loss Problemi ... . . . 3

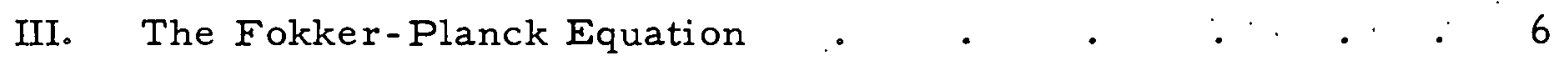

IV. Evaluation of the Expectation Values $\left\langle\Delta v_{j}\right\rangle$ and $\left\langle\Delta v_{i} \Delta v_{j}\right\rangle \cdot \quad$. 7

V. The Cylindrically Symmetric :Fokker-Planck:Equation . . . 1.3

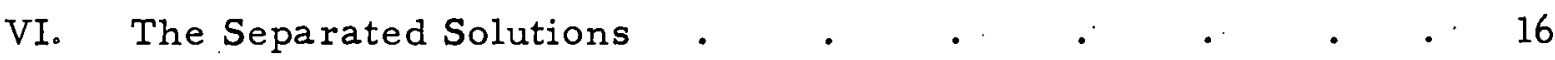

VII. Sëparated Solutions with a Source Function • . . . . . . 20

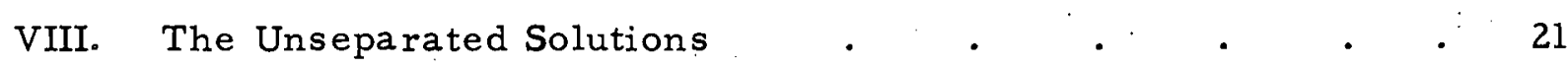

IX. Numerical Results of the End-Loss Calculations:

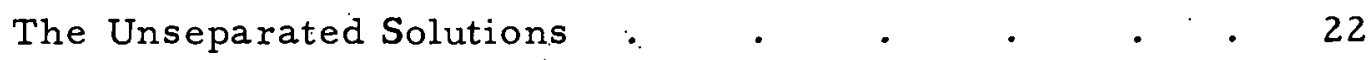

X. Numerical Results of the End-Loss Calculations:

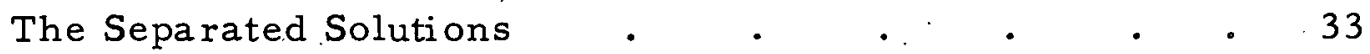

XI. Numerical Results of the End-Loss Calculations:

The Separated Solutions with a Source Function . . . 39

XII. Supplementary Calculations Based on the

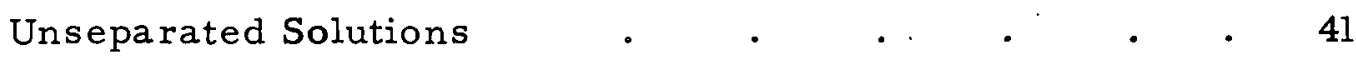

Relative Numbers of Particles in Certain Energy Ranges . 43

Rates of Charge-Exchange $\quad . \quad \ldots \quad . \quad . \quad . \quad 44$

Fusion Reaction Rates . . . . . . . . . 47

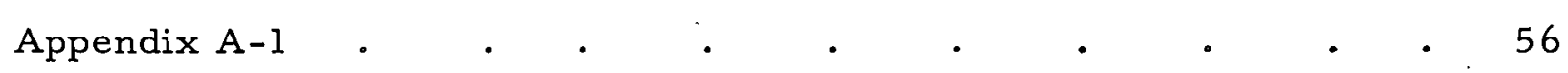

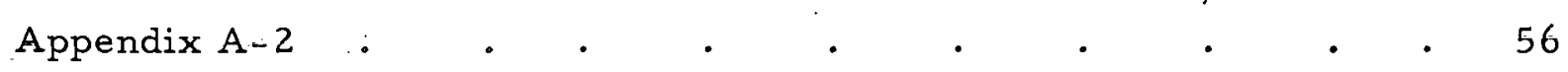

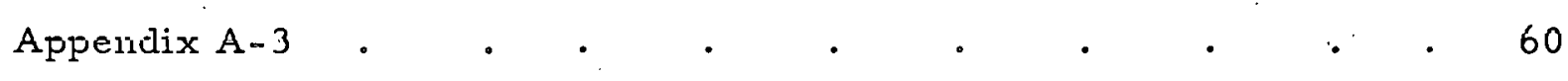


TABLE OF CONTENTS (Cont'd)

B - Computational Part . . . . . . . . . . . . . . 64

I. Numerical Methods Used . $\quad . \quad$. $\quad . \quad . \quad . \quad . \quad 65$

II. Boundary Conditions . . . . . . . . . . . . . . . . . . . 67

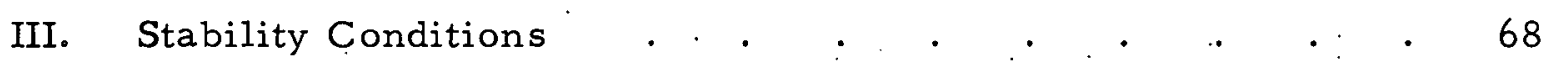

IV. Machine Calculations : . . : : . . . . . . . . . . 68

V. Special Boundary Conditions Used for a Coarse Grid . . . 71

VI.. Paiticle Density $\quad$. . . . . . . . . . . . 73

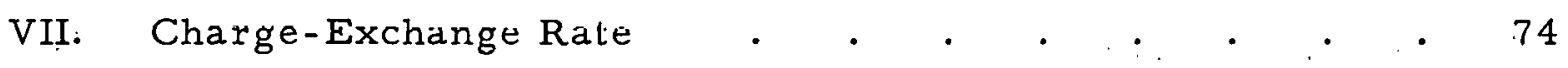

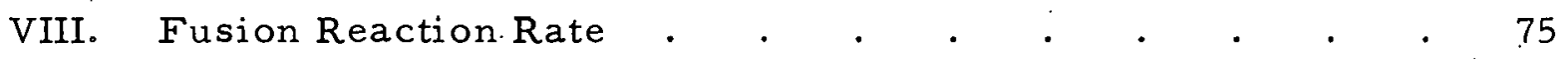

Appendix B-1 $\quad$ - $\quad$. 


\title{
END-LOSSES FROM MIRROR MACHINES
}

John E. Roberts and Marlene L. Carr

Lawrence Radiation Laboratory, University of California Livermore, California*

\begin{abstract}
This report describes a theoretical treatment of the loss of plasmas from the ends of magnetic mirror machines, based on the Fokker-Planck equation. The plasma losses are described in terms of the evolution in time of distribution functions.

Some supplementary calculations based on these distribution functions are also made. These include charge-exchange reaction rates and fusion reaction rates for the $\mathrm{DD}$ and $\mathrm{DT}$ reactions:
\end{abstract}

\section{A - PHYSICAL PART}

\section{INTROD UCTION}

For the past several years Dr. Richard F. Post and his research group have been studying a proposed device called the mirror machine, whose purpose is the production of controlled the rmonuclear power. The mirror. machine is pictured as containing a completely ionized heavy-hydrogen plasma heated to hundreds of millions of degrees. In the simplest conception of the device the containment of the plasma is to be accomplished by means of a solenoidal magnetic field modified by regions of increased field strength, the so-calied magnetic mirrors, at the ends of the machine.

The charged particles of the plasma move along the field lines in spiral trajectories between the two mirrors." At the mirrors some of them are

*Work was performed under auspices of the U. S. Atomic Energy Commission. 
reflected back into the central region while: others pass through and are lost out the ends of the machine.' Specifically, those particles are reflected whose spiral trajectories have a pitch angle $\theta$ in the central region of the machine that is greater than a certain critical angle determined by the mirror ratio $R$ (the ratio of the field strength in the mirror to the field strength in the central region). This critical angle is given by 1

$$
\sin \theta \geqslant \sin \theta_{R}=\frac{1}{\sqrt{R .}} \text {. }
$$

In this report we have assumed that the plasma is initially composed only of particles with pitch angles greater than the critical angle; such an initial state can be realized; for example, by injecting all the particles into the machine at the same angle. According to the simple adiabatic theory often applied to such containment problems, ${ }^{1}$ the particles would then remain in the machine indefinitely. However, their mutual Coulomb interactions can result in the loss of particles by means of scattering into trajectories that do not have reflections at the mirrors. ${ }^{2}$

The basic theory of this process has been worked out by Rosenbluth, MacDonald, and $\operatorname{Judd}^{3,4,5}$ and has been used by them and by other authors

$1_{R}$. F。 Post, UCRL-4231 (1954)。

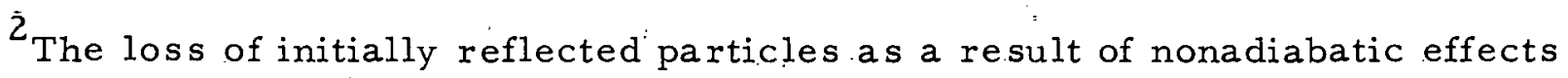
has been treated by Garren, Henrich, Northrop, Riddell, and Smith, TID-7536. (pt. 2) Book 1 of 2 (1957) and in Garren, Riddell, Smith, Bing, Roberts, Northrop, and Henrich, Proceedings of the Second United Nations International Conference on the Peaceful Uses of Atomic Energy (Geneva, 1958). (Also UCRL-8076). Broadly speaking, the chief nonadiabatic effect is a moderate increase of the critical pitch angle beyond the value given by Eq. (1)。

3 Judd, MacDonald, and Rosenbluth, W.ASH-289 (1955).

${ }^{4}$ M. N. Rosenbluth, TID-7503 (1955).

${ }^{5}$ Rosenbluth, MacDonald, and Judd, Phys. Rev. 107, 1 (1957). 
to obtain numerical estimates of the end-loss rates $6,7,8$ and to carry out a closely related relaxation calculation. 9 In this report we shall give a brief exposition of this theory and then review both old and new numerical results based upon it.

II. BASIC ASSUMPTIONS OF THE ROSENBLUTH-MACDONALD-JUDD THEORY IN THIS TREATMENT OF THE END-LOSS PROBLEM.

The loss rate through the mirrors can easily be calculated if we know the evolution in time of the particle distribution functions $f_{i}$, where the subscript i distinguishes between the various particle species. This evolution is described by the Boltzmann equation:

$$
\frac{\partial f_{i}}{\partial t}+\underline{v} \cdot \underline{\nabla} f_{i}+\frac{\underline{F}}{m} \cdot \underline{\nabla}_{v} f_{i}=\left(\frac{\partial f_{i}}{\partial t}\right)_{c},
$$

where $\left(\partial f_{i} / \partial t\right)_{c}$ denotes the time rate of change of $f_{i}$ resulting from collisions, and where $\underline{F}=e(\underline{E}+\underline{v} \times \underline{B})$.

Because the Boltzmann equation as it stands in Eq. (2) is too complicated for numerical treatment, various simplifying assumptions are mate in the Rosenbluth-MacDonald-Judd Theory.

These assumptions are:

1. 'l'he length of the machine is much larger than the length of the mirrors, so that most of the particle interactions take place in the central region. Consequently, we do not need to solve the Boltzmann

${ }^{6}$ Bing, Judd, MacDonald, and Rosenbluth, TID-7503 (1955).

${ }^{7}$ Garren, Riddell, Smith, Bing, Roberts, Northrop and Henrich, Proceedings of the Second United Nations International Conference on the Peaceful uses of Atomic.Energy (Geneva, 1958). (Also UC̣RL-8076).

${ }^{8}$ J. E. Roberts, UCRL-5132 (1958).

${ }^{9}$ MacDonald, Rosenbluth, and Chuck, Phys. Rev. 107, 350 (1957). 
equation in the mirror regions, where the dependence of the field on position would introduce great complications:

2. The radius of the machine is much larger than the Larmor radius of the particles. This means that the distribution functions are azimuthally and radially invariant; and, as is shown in Appendix $\dot{A}-1$, that $\underline{\mathrm{v}} \times \underline{\mathrm{B}} \cdot \nabla_{\mathrm{v}} \mathrm{f}_{\mathrm{i}}^{\mathrm{i}}=\dot{0}$.

3. The mean free path of the particles is much largei than the length of the machine, so that particles scattered into luss lrajecturies dicappear from the plasma immediately, even if the srattering occurs deep inside the machine. The distribution functions are therefore independent of position along magnetic field lines in the central region of the machine. This result, together with as sumption 2 and its consequences, implies that $\underline{\nabla} f_{i}=0$. It also implies that the electric field $\underline{E}$ is negligibly small throughout the central region.

It is now clear that Eq. (2) reduces to

$$
\frac{\partial f_{i}}{\partial t}=\left(\frac{\partial f_{i}}{\partial t}\right)_{c},
$$

which means that the evolution of the distribution functions can be calculated by evaluating the collision term of the Boltzmann equation. The ionic and electronic Boltzmann equations are coupled together by the collision terms, but the ion-electron collisions have a much smaller effect on the ion motions than the ion-ion collisions as long as $\mathrm{T}_{\mathrm{i}} / \mathrm{T}_{\mathrm{e}} \ll \mathrm{m}_{\mathrm{i}} / \mathrm{m}_{\mathrm{e}}, 10$ which is very large ( $T$ and $m$ designate temperature and particle mass). We can therefore

\footnotetext{
${ }^{10}$ This condition is easily deducible from Eqs. (29) and (35) of reference 15.
} 
neglect the ion-electron collisions and solve the ionic Boltzmann equation separately.

Even though the ion-electron collisions are negligible, the electrons do have three important effects. First, they cancel the space charge, making possible the vanishing of the electric field in the central region. Second, they shield the ions from each other, which results in an upper limit equal to the Debye shielding length for the ionic impact parameters. Third, they change the effective mirror ratio, as has been shown by Allan Kaufman, ${ }^{11}$ because if $\mathrm{T}_{\mathrm{i}}$ is not much greater thean $\mathrm{T}_{e}$. the electrons initially go out the ends faster than the ions, which creates a space charge and a resulting electric field. This field is restricted to the mirror regions and so does not spoil our earlier conclusion that $\underline{E}$ vanishes in the central region. Kaufman has shown that the electric field increases the critical loss angle for the ions and decreases it for the electrons. This process continues until the loss rates are the same for both ions and electrons. Kaufman's expressions for the effective mi rror ratios are

$$
\begin{aligned}
& R_{i}\left(W_{i}\right)=R /\left\{1+\frac{k T_{e}\left[4+\frac{3}{2} \ln \left(T_{i} / T_{e}\right)\right]}{W_{i}}\right\}, \\
& R_{e}\left(W_{e}\right)=R /\left\{1-\frac{k T_{e}\left[4+\frac{3}{2} \ln \left(T_{i} / T_{e}\right)\right]}{W_{e}}\right\},
\end{aligned}
$$

where $W$ is the energy of the particular ion or electron. According to Eq̣s. (4), the effective mirror ratio is a function of W. Although our calculation assumes $R$ to be independent of $W$, it becomes valid in the limit where $\mathrm{T}_{\mathrm{e}} \ll \mathrm{T}_{\mathrm{i}} \cdot$. Furthermore, we shall see later on that the los s rate is not especially sensitive to the mirror ratio if the mirror ratio is sufficiently large. We should the refore expect our calculations to be approximately

${ }^{11}$ A. N. Kaufma n, TID-7520 (Pt. 2) (1956), p. 3'87. 
valid if the electron temperature is small (though not so small as to make ion-electron collisions important) or if the mirror ratio is large.

\section{THE FOKKER-PLANCK EQUATION}

In plasmas of thermonuclear interest the particle densities are so low $\left(\sim 10^{11}\right.$ to $10^{14}$ particles $\left./ \mathrm{cm}^{3}\right)$ and the particle energies so high $(\sim 10 \mathrm{kev}$ to $1 \mathrm{Mev}$ ) that the mean potential energy between the particles is much less than their mean kinetic energy. Under these conditions the cross section for the deflection of a particle through a large angle by means of a series of smallangle Coulomb interactions is about twenty or thirty times as large as the cross section for such a deflection by means of a single large-angle interaction. ${ }^{12}$ It is therefore the distant rather than the close encounters between particles that are the most important; most of the scattering of single particles is produced by encounters for which the impact parameter is much larger than the mean interparticle distance.

The collision term in the Boltzmann equation is usually taken to be the integral equation: ${ }^{13}$

$$
\left(\frac{\partial f(1)}{\partial t}\right)_{c}=-\int[f(1) f(2)-f(3) f(4)] \sigma(\Omega) d \Omega d v_{2}
$$

in which the first term is the probability that two particles with initial velocities $v_{1}$ and $v_{2}$ will undergo a collision leading to final velocities $\mathrm{v}_{3}$ and $\mathrm{v}_{4}$, the second term is the probability of the corresponding inverse collision, $\sigma(\Omega)$ is the differential scattering cross section, and $d \Omega$ is the differential of solid angle. (The velocities $\dot{v}_{3}$ and $v_{4}$ are of course functions

12 Lyman Spitzer, Jr., Physics of Fully Ionized Gases (Interscience Publishers Inc., New York, 1956), pp. 66-78.

13 See for example S. Chapman and T. G. Cowling, The Mathematical Theory of Non-Uniform Gases (Cambridge, London, 1939). 
of $\mathrm{v}_{1}, \mathrm{v}_{2}$ and the two angles involved in $\Omega$.) This expression is derived from the premise that the encounters consist of isolated two-particle interactions, uninfluenced by the presence of other particles, as is the case when the particles interact through short-range forces. But with Coulomb forces and with low densities and high temperatures we have seen that the most i mportant collisions are those with impact parameters much larger than the mean interparticle distance, so that most of the time each particle is interacting with many other particles simultaneously. The net force of interaction is then continuous, small, and rapidly fluctuating; in fact, many fluctuations take place during periods in which the particle velocities change only infinitesimally. Under these conditions it is more appropriate to describe the collisions by a diffusion-like term than by an integral of the form (5). Such a description is given by the Fokker-Planck equation ${ }^{14,15}$

$$
\left(\frac{\partial f}{\partial t}\right)_{c}=-\sum_{i=1}^{3} \frac{\partial}{\partial v_{i}}\left(f\left\langle\Delta v_{i}\right\rangle\right)+\frac{1}{2} \sum_{i, j} \frac{\partial^{2}}{\partial v_{i} \partial v_{j}}\left(f\left\langle\Delta v_{i} \Delta v_{j}\right\rangle\right),
$$

in which $v_{i}$ is the $i^{\text {th }}$ component of particle velocity in Cartesian coordinates, $\Delta v_{i}$ is the change in $v_{i}$ resulting from the interactions during a unit of time, and the brackets 〈 indirate the expectation value of the enclosed expressions. The unit of time is taken to be large compared with the fluctuation time of the interaction and small compared with the macroscopic time scale.

IV. EVALUATION OF THE EXPECTATION VALUES

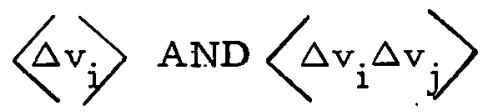

Consider a single particle p (the "scattered" particle) for which we wïsh to calculate the expectation values $\left\langle\Delta v_{i}\right\rangle$ and $\left\langle\Delta v_{i} \Delta v_{j}\right\rangle$. We simply average

${ }^{14}$ S. Chandrasekhar, Rev. Mod. Phys. 15, 1 (1943), pp. 31-33.

${ }^{15}$ Gasiorowicz, Neumann, and Riddell, Phys. Rev. 101, 922 (1956). 
$\Delta v_{i}$ and $\Delta v_{i} \Delta v_{j}$ over all velocities of the particles that interact with $p$ (the "scattering" particles) and over all scattering angles as follows: 5

$$
\left\langle\Delta \dot{v}_{i}\right\rangle=\int f\left(\underline{v}^{\prime}\right) d \underline{v}^{r} \int d \Omega \sigma\left(v_{r}, \dot{\Omega}\right) v_{r} \Delta v_{i}
$$

and

$$
\left\langle\Delta v_{i} \Delta v_{j}\right\rangle=\int f\left(\underline{v}^{\prime}\right) d \underline{v}^{\prime} \int d \Omega \sigma\left(v_{r}, \Omega\right) v_{r} \Delta v_{i} \Delta v_{j}
$$

where $\underline{v}^{\prime}$ is the velocity of the scattering particle, $v_{r}=\left|\underline{v}^{\prime}-\underline{v}\right|$ is its speed relative to the scattered particle, $\Omega$ is the scattering solid angle, and $\sigma\left(v_{r}, \Omega\right)$ is the differential scattering cross section.

We average $\Delta v_{i}$ and $\Delta v_{i} \Delta v_{j}$ over the scattering angles in the centerof-mass system, because in that system collisions result merely in simple rotations of the relative velocity vector. 'In the averaging we also use the Coulomb differential scattering cross section:

$$
\sigma\left(v_{r}, \Omega\right)=\left(e^{4} / 4 m_{r}^{2} v_{r}^{4}\right) \sin ^{-4} \frac{\theta}{2},
$$

where $m_{r}$ is the reduced mass and $\theta$ is the scattering angle in the centerof-mass system. We then transform to the laboratory frame and average over the velocities to obtain expressions for the expectation values $\left\langle\Delta . v_{i}\right\rangle$ and

$$
\left\langle\Delta v_{i} \Delta v_{j}\right\rangle
$$

In all the scattering calculations of this paper we assume that both the scattered and the scattering particles are the same kind. Then the velocity of the scattered particle is given by

$$
\underline{v}=\underline{v}+\frac{1}{2} \underline{v}
$$

where $v_{r}$ is the velocity of the scattered particle relative to the scattering particle and $\underline{V}$ is the velocity of the center-of-mass. $\underline{V}$ remains unchanged in a collision so that

$$
\Delta \underline{v}=\frac{1}{2} \cdot \Delta \underline{v_{r}} .
$$


$-9-$

UCRL 5651-T

We choose the unit vectors $\underline{\xi}, \underline{\eta}, \underline{\xi}$ of the center-of-mass frame to be

$$
\begin{aligned}
& \underline{\xi}=\left(\frac{v_{r i}}{v_{r}}, \frac{v_{r j}}{v_{r}}, \frac{v_{r k}}{v_{r}}\right)=\frac{v_{r}}{v_{r}},
\end{aligned}
$$

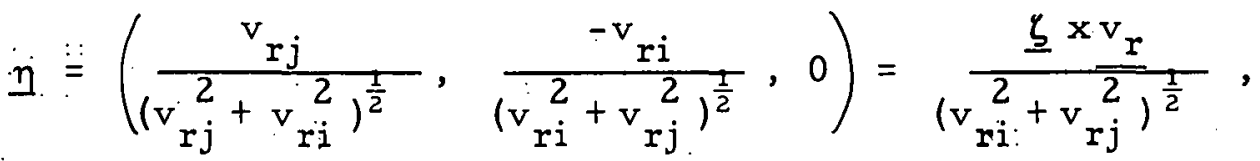

$$
\begin{aligned}
& \underline{\xi}=\left(\frac{v_{r i} v_{r k}}{v_{r}\left(v_{r i}^{2}+v_{r j}^{2}\right)^{\frac{1}{2}}}, \frac{v_{r j} v_{r k}}{v_{r}^{\left(v_{r i}^{2}+v_{r j}^{2}\right)^{\frac{1}{2}}}}, \frac{-\left(v_{r i}^{2}+v_{r j}^{2}\right)^{\frac{1}{2}}}{v_{r}}\right)=\underline{\xi} \times \underline{\eta},
\end{aligned}
$$

and then obtain the $\underline{\xi}, \underline{\eta}$, and $\underline{\xi}$ components of $\Delta \underline{v}$ from an inspection of Fig. 1:

$$
\begin{aligned}
& \Delta v_{\xi}=\frac{1}{2} \Delta v_{r \xi}=-v_{r} \sin ^{2} \frac{\theta}{2}, \\
& \Delta v_{\eta}=\frac{1}{2} \Delta v_{r \eta}=-v_{r} \sin \frac{\theta}{2} \cos \frac{\theta}{2} \cdot \cos \phi, \\
& \Delta v_{\zeta}=\frac{1}{2} \Delta v_{r \zeta}=v_{r} \sin \frac{\theta}{2} \cos \frac{\theta}{2} \sin \phi .
\end{aligned}
$$

We then integrate Eqs. (12), (13), and (14) over $\theta$ and $\phi$ and obtain

$$
\begin{aligned}
\left\langle\Delta v_{\xi}\right\rangle_{\theta, \phi i} & =\int \mathrm{d} \Omega \sigma\left(\mathrm{v}_{\mathrm{r}}, \Omega\right)_{\mathrm{r}} \Delta \mathrm{v}_{\xi} \\
& =\int_{\theta=0}^{\theta=\pi} \int_{\phi=0}^{\phi=2 \pi} \sin \theta \mathrm{d} \theta \mathrm{d} \phi\left(\mathrm{e}^{4} / 4 \mathrm{~m}_{\mathrm{r}}^{2} \mathrm{v}_{\mathrm{r}}^{4}\right) \cdot \sin -4 \frac{\theta}{2} \cdot\left(\mathrm{v}_{\mathrm{r}} \sin ^{2} \frac{\theta}{2}\right) \\
& =-\left.\left(2 \pi \mathrm{e}^{4} / \mathrm{m}_{\mathrm{r}}^{2} \mathrm{v}_{\mathrm{r}}^{2}\right) \ln \sin \frac{\theta}{2}\right|_{0} ^{\pi}
\end{aligned}
$$

and

$$
\left\langle\Delta v_{\eta}\right\rangle_{\theta, \phi^{\prime}}=\left\langle\Delta v_{\xi}\right\rangle_{\dot{\theta}, \phi .}=0 .
$$

It is apparent from Eq. (15) that $\left\langle\Delta v_{\xi}\right\rangle, \theta$, will diverge unless we cut off the integration at small scattering angles. We make the cutoff at a minimum 


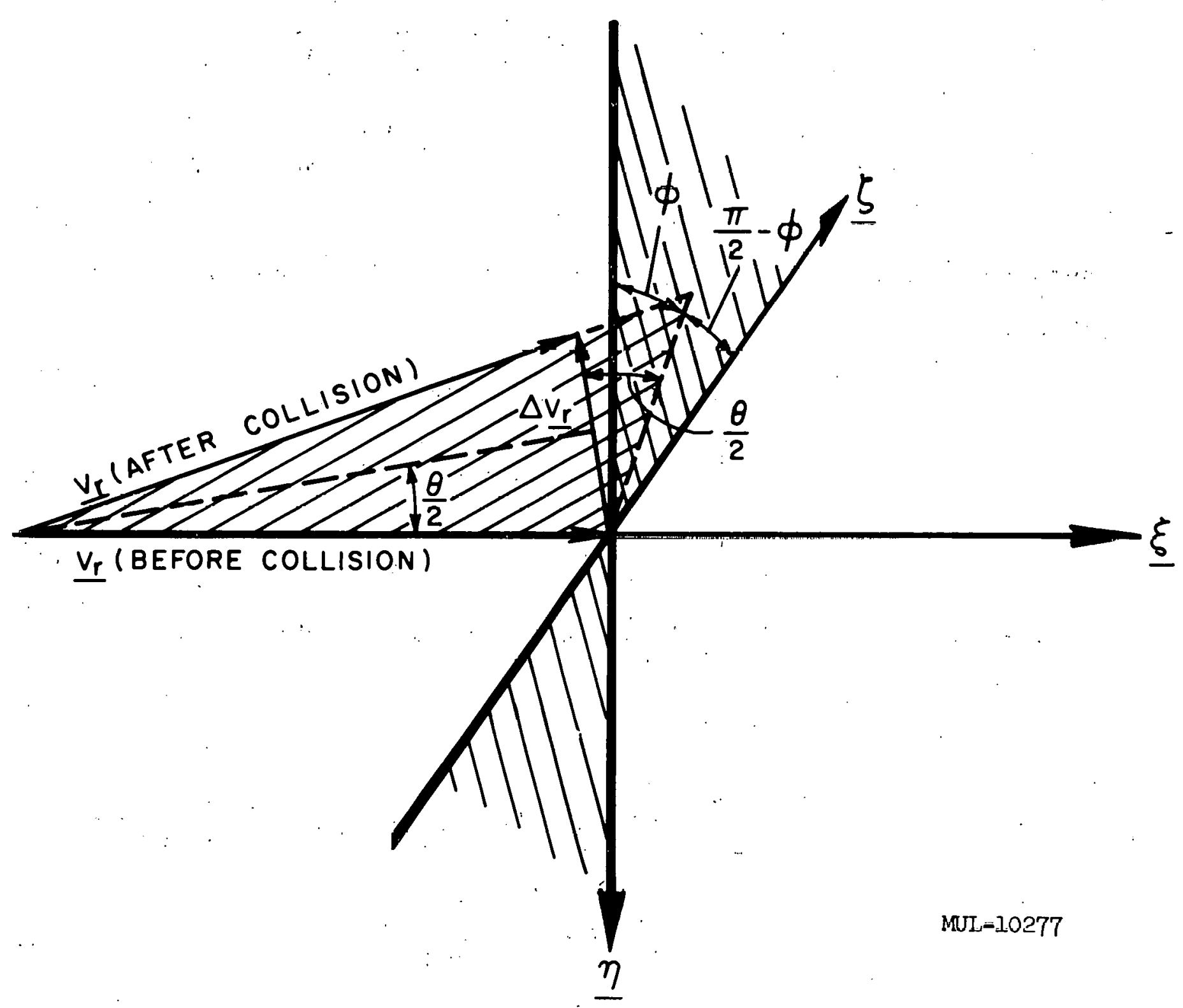

Fig. 1. Diagram showing kinematics of an elastic scattering in the center-of-mass Cartesian system. 
angle $\theta_{m}$ that corresponds to a collision parameter equal to the Debye length $\lambda_{D}$. We assume that interactions between particles at distances greater than $\lambda_{D}$ are completely shielded by the plasma. We now have

$$
\left\langle\Delta v_{\xi}\right\rangle_{\theta, \phi}=-\left(2 \pi \mathrm{e}^{4} / \mathrm{m}_{\mathrm{r}}^{2} \mathrm{v}_{\mathrm{r}}^{2}\right) \ln \frac{2}{\theta_{\mathrm{m}}} .
$$

We set $D$ equal to $2 / \theta_{\mathrm{m}}$ and evaluate it as described by Spitzer, ${ }^{12}$ obtaining

$$
\left.D \equiv \frac{2}{\theta_{m}}=\overline{\left(m_{r} v_{r}^{2}\right.} \lambda_{D} / e^{2}\right)
$$

where the bar denotes "mean value of" and $\lambda_{D}=\left(k T / 4 \pi n e^{2}\right)^{1 / 2}, n$ being the electron density.

The quantity $D$, which is proportional to the ratio of the Debye length $\lambda_{D}$ to the mean classical distance of closest approach $e^{2} / \frac{1}{2} \cdot \overline{m_{r} v_{r}^{2}}$, is a very large number in the cases of thermonuclear interest - so large, in fact, that $\ln D$ will be approximately twenty to thirty. ${ }^{12}$ Since $D$ appears only logarithmically in the equations for $\left\langle\Delta v_{i}\right\rangle$ and $\left\langle\Delta v_{i} \Delta v_{j}\right\rangle$, the precise value of $\mathrm{D}$ is relatively unimportant and we take both $\mathrm{kT}$ and $\mathrm{m}_{\mathrm{r}} \mathrm{v}_{\mathrm{r}}^{2}$ to be proportional to the mean energy of the plasma.

We evaluate the $\left\langle\Delta v_{\xi, \eta, \eta},{ }^{\Delta v_{\xi}}, \eta, \zeta\right\rangle_{\theta, \phi}$ similarly by integrating $\Delta \mathrm{v}_{\xi, \eta, \zeta}{ }^{\Delta \mathrm{v}_{\xi}, \eta, \zeta}$ over $\mathrm{d} \Omega$ as indicated in Eq. (8). The off-diagonal terms vanish because of the integrations over $\phi$. Integration of the diagonal terms yields

$$
\begin{aligned}
& \left\langle\Delta v_{\xi} \Delta v_{\xi}\right\rangle_{\theta, \phi}=\pi e^{4} / m_{r}^{2} v_{r}, \\
& \left\langle\Delta v_{\eta} \Delta v_{\eta}\right\rangle_{\theta, \phi}=\left\langle\Delta v_{\zeta} \Delta v_{\zeta}\right\rangle_{\theta, \phi}=\left(\pi e^{4} / m_{r}^{2} v_{r}\right)\left[(\ln D)-\frac{1}{2}\right] .
\end{aligned}
$$

Ignoring the nonlogarithmic terms with respect to $\ln D$, we obtain

$$
\begin{aligned}
& \left\langle\Delta v_{\xi} \Delta v_{\xi}\right\rangle_{\theta, \phi}=0 \\
& \left\langle\Delta v_{\eta} \Delta v_{\eta}\right\rangle_{\theta, \phi}=\left\langle\Delta v_{\zeta} \Delta v_{\zeta}\right\rangle_{\theta, \phi}=\left(\pi e^{4} / m_{r}{ }^{2} \cdot v_{r}\right) \text { in } D .
\end{aligned}
$$


$-12 .-$

UCRL 5651-T.

We now transform to the laboratory frame of reference by means of the relations

$$
\begin{aligned}
& \left\langle\Delta v_{i}\right\rangle_{\theta, \phi}=\sum_{a=\xi, \eta, \xi}(\underline{a} \cdot \underline{i})\left\langle\Delta v_{a}\right\rangle_{\theta, \phi}, \\
& \left\langle\Delta v_{i} \Delta v_{j}\right\rangle_{\theta, \phi}=\sum_{a}(\underline{a} \cdot \underline{i}\rangle_{a}(\underline{j})\left\langle\Delta v_{a}^{2}\right\rangle_{\theta, \phi},
\end{aligned}
$$

obtaining

$$
\begin{aligned}
& \left\langle\Delta v_{i}\right\rangle_{\theta, \phi}=-\left(2 \pi e^{4} v_{i \cdot i} / m_{i \cdot}^{2} v^{3}\right) \ln \cdot \dot{D}, \\
& \left\langle\Delta v_{i} \Delta v_{j}\right\rangle_{\theta, \phi}=\left(\pi e^{4} \ln D / m_{r}^{2} v_{r}^{3}\right)\left(v_{r}^{2} \delta_{i j}-v_{r i} v_{r j}\right) .
\end{aligned}
$$

We simplify Eqs.(20) by noting that $v_{r}=\sqrt{\sum\left(v_{i}-v_{i}^{\prime}\right)^{2}}$ and obtain

$$
\begin{aligned}
& \left\langle\Delta v_{i}\right\rangle_{\theta, \phi}=2 \Gamma \frac{\partial}{\partial v_{i}} \cdot \frac{1}{v_{r}} \cdots, \\
& \left\langle\Delta v_{i} \Delta v_{j}\right\rangle_{\theta, \phi}=\Gamma \frac{\partial^{2} v_{r}}{\partial v_{i} \partial v_{j}},
\end{aligned}
$$

where $\Gamma=\pi e^{1} \ln D / m_{r}^{2}=4 \pi e^{1} \ln D / m^{2}$.

We then substitute Eq. (21) and Eq. (7) and (8) and get

$$
\begin{aligned}
& \left\langle\Delta v_{i}\right\rangle=\int d \underline{v}^{\prime} f\left(\underline{v}^{\prime}\right)\left\langle\Delta v_{i}\right\rangle_{\theta, \phi}^{=}=2 \Gamma \frac{\partial h}{\partial v_{i}}, \\
& \left\langle\dot{\Delta} v_{i} \Delta v_{j}\right\rangle=\int d \underline{v}^{\prime} f\left(\underline{v}^{\prime}\right\rangle\left\langle\Delta v_{i} \Delta v_{j}\right\rangle_{\theta, \phi}=\Gamma \frac{\partial^{2} g}{\partial v_{i} \partial v_{j}},
\end{aligned}
$$

where

$$
\begin{aligned}
& h \underline{v})=\int d \underline{v}^{\prime} f\left(\underline{v}^{\prime}\right) / v_{r}=\int d \underline{v}^{\prime} \cdot f\left(\underline{v}^{\prime}\right) / \sqrt{\sum\left(v_{i}-v_{i}^{i}\right)^{2}}, \\
& g(\underline{v})=\int d \underline{v}^{\prime} f\left(\underline{v}^{\prime}\right) v_{r}=\int d \underline{v}^{\prime} f\left(\underline{v}^{\prime}\right) \sqrt{\sum\left(v_{i}-v_{i}^{\prime}\right)^{2}} .
\end{aligned}
$$


And finally we substitute Eqs. (22) and (23) into Eq. (6) and obtain

$$
\frac{i}{\Gamma}\left(\frac{\partial f}{\partial t}\right)_{c}=-2 \sum_{i=1}^{3} \frac{\partial}{\partial v_{i}}\left(f \frac{\partial h}{\partial v_{i}}\right)+\frac{1}{2} \sum_{i, j} \frac{\partial^{2}}{\partial v_{i} \partial v_{j}}\left(f \frac{\partial^{2} g}{\partial v_{i} \partial v_{j}}\right) \text {, }
$$

which expresses the Fokker-Planck equation as an integro-differential equation explicit in terms of the distribution function.

\section{THE CYLINDRICALLY SYMMETRIC FOKKER-PLANCK EQUATION}

In applying Eq. (26) to the end-loss problem we may capitalize on the cylindrical symmetry of the mirror machine to effect some simplification. We introduce the coordinates $v, \mu$, $\phi$ into Eq. (26), where $v=\sqrt{\sum_{i} v_{i}^{2}}$ and $\mu=v_{z} / v=\cos \theta$ (as before, $\theta$ is the pitch angle between the particle velocity and the magnetic field axis, and $\phi$ is the azimuthal angle of the velocity), and obtain an equation in terms of $v$ and $\mu$ :

$$
\begin{aligned}
\frac{1}{\Gamma}\left(\frac{\partial f}{\partial t}\right)_{c}= & 4 \pi f^{2}+\frac{1}{2} g_{v v} f \cdot+\left[\frac{\left(1-\mu^{2}\right)}{v^{2}} g_{\mu v}-\frac{\left(1-\mu^{2}\right)}{v^{3}} g_{\mu}\right] f_{\mu v} \\
+ & {\left[\frac{\left(1-\mu^{2}\right)^{2}}{2 v^{4}} g_{\mu \mu}-\frac{\mu\left(1-\mu^{2}\right)}{2 v^{2}} g_{\mu}+\frac{\left(1-\mu^{2}\right)}{2 v^{3}} g_{v}\right] f_{\mu \mu} } \\
& +\left[\frac{\left(1-\mu^{2}\right)}{2 v^{3}} g_{\mu \mu}-\frac{\mu}{v^{3}} g_{\mu}+\frac{1}{v^{2}} g_{v}\right] f_{v} \\
& +\left[-\frac{\mu\left(1-\mu^{2}\right)}{2 v^{4}} g_{\mu \mu}+\frac{1}{v^{4}} g_{\mu}-\frac{\left(1-\mu^{2}\right)}{v^{3}} g_{\mu v}-\frac{\mu}{v^{3}} g_{v}\right] f_{\mu},
\end{aligned}
$$

in which $f_{\mu v}=\frac{\partial^{2} f}{\partial \mu \partial v}$, etc.

The transformation from Eq. (26) to Eq. (27) may be accomplished by the extremely tedious process of directly substituting spherical coordinates into. Eq. (26) or by the less involved, but still tedious, method given in reference 5. In going from Eq. (26) to Eq. (27) we also use rélalions obtained by differentiating Eqs. (24) and (25) and noting a formal similarity 
with potential theory:

$$
\begin{aligned}
\frac{1}{2} \nabla_{\mathrm{v}}^{2} \mathrm{~g} & =\sum_{\mathrm{i}} \frac{\partial^{2} \mathrm{~g}}{\partial \mathrm{v}_{i}^{2}}=\mathrm{h} \\
\nabla_{\mathrm{v}}^{2} \mathrm{~h} & =-4 \pi f
\end{aligned}
$$

Because solutions independent of specific injection energies or specific particle densities were desired, Eq. (27) was converted into the form

$$
\begin{aligned}
& \left(\frac{\partial F}{\partial T}\right)_{c}=4 \pi F^{2}+\frac{1}{2} G_{x x} F x+\left[\frac{\left(1-\mu^{2}\right)}{x^{2}} G_{\mu x}-\frac{\left(1-\mu^{2}\right)}{x^{3}} G_{\mu}\right] F_{\mu x} \\
& +\left[\frac{\left(1-\mu^{2}\right)^{2}}{2 x^{4}} G_{\mu \mu}-\frac{\mu\left(1-\mu^{2}\right)}{2 x^{4}} G_{\mu}+\frac{\left(1-\mu^{2}\right)}{2 x^{3}} G_{x}\right] F_{\mu \mu} \\
& +\left[\frac{\left(1-\dot{\mu}^{2}\right)}{2 x^{3}} G_{\mu \mu}-\frac{\mu}{x^{3}} G_{\mu}+\frac{1}{x^{2}} G_{x}\right] F_{x} \\
& +\left[\because \frac{\mu\left(1-\mu^{2}\right)}{2 x^{4}} G_{\mu \mu}+\frac{1}{x^{4}} G_{\mu}-\frac{\left(1-\mu^{2}\right)}{x^{3}} G_{\mu x}-\frac{\mu}{x^{3}} G_{x}\right] F_{\mu}
\end{aligned}
$$

by the substitition of

$$
\mathrm{x}=\frac{\mathrm{v}}{\mathrm{v}_{\mathrm{o}}}, \quad F=\frac{\mathrm{f}}{\mathrm{A}}, \mathrm{G}=\frac{\mathrm{g}}{\mathrm{A}}, \text { and } \tau=\Gamma \mathrm{At}
$$

where $v_{0}, f$, and A are defined as follows: $v_{0}$ is the speed of the particles at the injection energy $E$ and is given by

$$
\mathrm{v}_{\mathrm{o}}=\sqrt{2 \mathrm{E} / \mathrm{m}} \text {. }
$$

The distribution function $f$ is defined in such a way that

$$
\int_{0}^{\infty} \int_{-\mu_{R}}^{\mu_{R}} \int_{0}^{2 \pi} \dot{f}(\mu, v) d \phi d \mu v^{2} d v=n
$$

where $\mathrm{n}$ is the particle density...A is a normalizing factor such that 


$$
\begin{aligned}
A & =n /\left[\int_{0}^{\infty} \int_{-\mu}^{\mu} R \int_{0}^{2 \pi} F(\mu, v) d \phi d \mu v^{2} \cdot d v\right] \\
& =n /\left[2 \pi v_{0}^{3} \int_{0}^{\infty} \int_{-\mu_{R}}^{\mu} F(\mu, x) d \mu x^{2} d x\right] .
\end{aligned}
$$

By means of Eqs. (31), (32), and (34), solutions of Eq. (30) may be interpreted in terms of actual elapsed time as a function of the initial particle density $\mathrm{n}_{\mathrm{o}}$, the initial distribution function $F_{0}$, and the injection energy $E$.

The different numerical value of the integral $\int_{0}^{\infty} \int_{-\mu_{R}}^{\mu} F_{0}(\mu, x) d \mu x^{2} d x$ that arises from each different initial distribution function ${ }^{-\mu_{R}} F_{0}$ requires a different normalization factor $A$ and consequently a different conversion factor from $T$ to actual time $t$ for each initial distribution that we consider. However, to facilitate the direct comparison of elapsed times in the various results presented in Part A of this report, all. $\tau^{\prime}$ s have been adjusted so that they may be converted to actual time by means of the relation

$$
t=\frac{1.000 \times 10^{10}}{\left(1.000+0.1019 \log E_{e}-0.0340 \log n_{o}\right)^{-0.00}} \cdot \frac{E^{3 / 2}}{n_{0}} \tau
$$

where $\mathrm{t}$ is in seconds, $\mathrm{n}_{\mathrm{o}}$ is in particles $/ \mathrm{cm}^{3}$, and $\mathrm{E}$ and $\mathrm{E}_{\mathrm{e}}$ (the mean electron energy) are in kilovolts. The $T$ in Eq. (35) is not the same as any of the $\tau^{\prime} s$ used in the actual computational work discussed in Part B, which were obtained directly from Eqs. (31), (32), and (34). A derivation of the relationship of $t$ to the various $\tau^{\prime}$ 's, which differ only by numerical factors from Eq. (35), is given in Appendix A-2.

Equation (30) was then solved numerically subject to the conditions $F(\mu, x)$ symmetric in $\pm \mu$, and $F(\mu, x)=0$ for $\mu \geq_{R}$, where from $E q$. (1) 


$$
\mu_{R}=\cos \theta_{R}=\sqrt{(R-1) / R} \text {. }
$$

Most of the calculations were made with an initial distribution substituted into.Eq. (30), but some were also made with Eq. (30) modified by the addition of a source function in order to study the effects of continuously injecting plasma into a mirror machine.

Equation (30) was solved by two different methods, one of which was morc accurate than the other. The more accurate calsuluations will be referred to as the unseparated solutions, and the less accurate calculations as the separated solutions. The results from both methods will be discussed because the major part of the less accurate results were not recalculated by the more accurate method, and because those results that were recalculated were in qualitative agreement. Until an IBM Model 704 digital computing machine became available, the time requirement for an unseparated numerical solution of Eq. (30) was prohibitive.

\section{THE SEPARATED SOLUTIUNS}

In the separated solutions the integral $G(\mu, x)=\int F x_{r} d x^{\prime}$ was assumed to be independent of $\mu$. This assumption was intended to imply that $F\left(\underline{x}^{\prime}\right)$ was independent of $\mu^{\prime}$ only for the integral $G$. Then $G_{\mu}, G_{\mu \mu}$, and $G_{\mu x}$ were all equal to zero, and Eq. (30) became

$$
\left(\frac{\partial F}{\partial T}\right)_{c}=4 \pi F^{2}+\frac{1}{2} G_{x \times x} F_{x x}+\frac{1}{x^{2}} G_{x} F_{x}+\left[\left(1-\mu^{2}\right) F_{\mu \mu}-2 \mu F{ }_{\mu}\right] \frac{G_{x}}{2 x^{3}} \text {. }
$$

The further assumptions we re made that $F(\mu, x, \tau)=M(x, \tau) S(\mu)$ and that $F^{2}=M^{2} S \times 1$ to permit a separation of variables:

$$
\frac{2 x^{3}}{G_{x}}\left[\frac{1}{M}\left(\frac{\partial M}{\partial \tau}\right)_{c}-4 \pi M^{2}-\frac{1}{2} \cdot \frac{G_{x x}}{M} M_{x x}-\frac{1}{x^{2}} \cdot \frac{G_{x}}{M} M_{x}\right]=\left[\left(1-\mu^{2}\right) S_{\mu \mu}-2 \mu S_{\mu}\right] \frac{1}{S} \text {. }
$$


Setting the constant of separation equal to $-\lambda$ yielded

and

$$
\left(\frac{\partial M}{\partial \tau}\right)_{C}=4 \pi M^{2}+\frac{1}{2} G_{x x} M_{x x}+\frac{1}{x^{2}} G_{x} M_{x}-\frac{G_{\dddot{x}}}{2 x^{3}} \lambda M
$$

$$
\left[\left(1-\mu^{2}\right) S_{\mu \mu}-2 \mu S_{\mu}\right]=-\lambda S,
$$

which is Legendre's Equation, where $\lambda=v(v+1)$. :

For integral values of $v$.Eq. (40) may be solved in terms of a linear combination of Legendre polynomials of the first and second kinds. Application of the boundary conditions, with the additional requirement that $S(0)=1$, gives the result that for $v$ even the solution to Eq. $(40)$ is the appropriately normalized Legendre polynomial of the first kind, while for $v$ odd it is the appropriately normalized Legendre polynomial of the second kind. Where $S(\mu)=0$ (i. e., at the first root of each polynomial), $\mu=\mu_{R}$, and hence through Eq. (36) there is a relation between $\nu$ or $\lambda$ and the mirror ratio, $R$.

Táble 1. A COMPARISON OF THE SEPARATION CONSTANT $\lambda$

\begin{tabular}{|c|c|c|c|c|c|c|}
\hline$\lambda$ & $v$ & $\begin{array}{l}\text { Solution to } \\
\text { Eq. }(40)\end{array}$ & $\begin{array}{l}\text { First root } \\
\text { of solution } \\
\mu_{R}\end{array}$ & $R=\frac{1}{1-\mu_{R}^{2}}$ & $\frac{1}{\log R}$ & \\
\hline 0 & 0 & $P_{0}(\mu)$ & --- & $-\cdots$ & $\ldots$ & \\
\hline 2 & 1 & $(-1) Q_{1}(\mu)$ & 0.83 & 3.27 & 1.95 & \\
\hline 6 & 2 & $(-2) P_{2}(\mu)$ & 0.58 & 1.51 & 5.59 & 1 \\
\hline 12 & 3 & $\left(\frac{3}{2}\right) \Omega_{3}(\mu)$ & 0.43 & 1. 23 & 11.3 & \\
\hline 20 & 4 & $\left(\frac{8}{3}\right) P_{4}(\mu)$ & 0.34 & 1.13 & 18.7 & \\
\hline 30 & 5 & $\left(-\frac{15}{8}\right) Q_{5}(\mu)$ & 0.28 & 1.08 & 30.3 & \\
\hline
\end{tabular}
WITH THE RECIPROCAL OF LOG R 
In Table 1, comparison of $\lambda$ with the corresponding. mirror ratio shows that $\lambda$. is approximated within about ten percent or less by the relation

$$
\lambda \approx \frac{1}{\log R}
$$

wherelog denotes logarithm to the base 10. Because most of the mirror ratios considered here corresponded to $\lambda$ 's that were' not related to integral-välued v's, Eq. (41) wa's used to give interpalated or extrapolated values of $\lambda$. For these values of $\lambda, S(\mu)$ was represented by an approximate solution,

$$
S(\mu)=a\left\{1-\exp \left[-\beta\left(\mu_{R}-|\mu|\right)\right]\right\},
$$

where $a=\left(1-e^{-\beta \mu_{R}}\right)^{-1}$. Equation (42) was approximately fitted to the solution of Eq. (40) by matching derivatives at $\mu_{R}$ :

$$
\begin{aligned}
& S(\mu)_{\mu}=\mu_{R}=0 \\
& \left(\frac{d S}{d \mu}\right)_{\mu-\mu_{R}}=-a \beta \exp -\beta\left(\mu_{R}-|\mu|\right) \mid \begin{array}{c}
\because \ldots \ldots . . . \\
|\mu|=\mu_{K}=-\pi \beta .
\end{array} \\
& \left(\frac{d^{2} s}{d \mu^{2}}\right)_{\mu=\mu_{R}}=-a \beta^{2} .
\end{aligned}
$$

Substitution of Eqs. (43), (44), and (45) into Eq. (40) gave

$$
\left(1-\mu_{R}\right\}\left(-a \beta^{2}\right)+2 \mu_{\Omega} a \beta+0=0 \text {, }
$$

from which

$$
\beta=2 \mu_{R} /\left(1-\mu_{R}^{2}\right)=2 \sqrt{R(R-1)}
$$

The approximate angular distribution function $S(\mu)$ could then be represented as a function of the mirror ratio $R$ by the substitution of Eq. (47) into Eq. (42). Figure 2 is a comparis on of the approximate angular solution given 


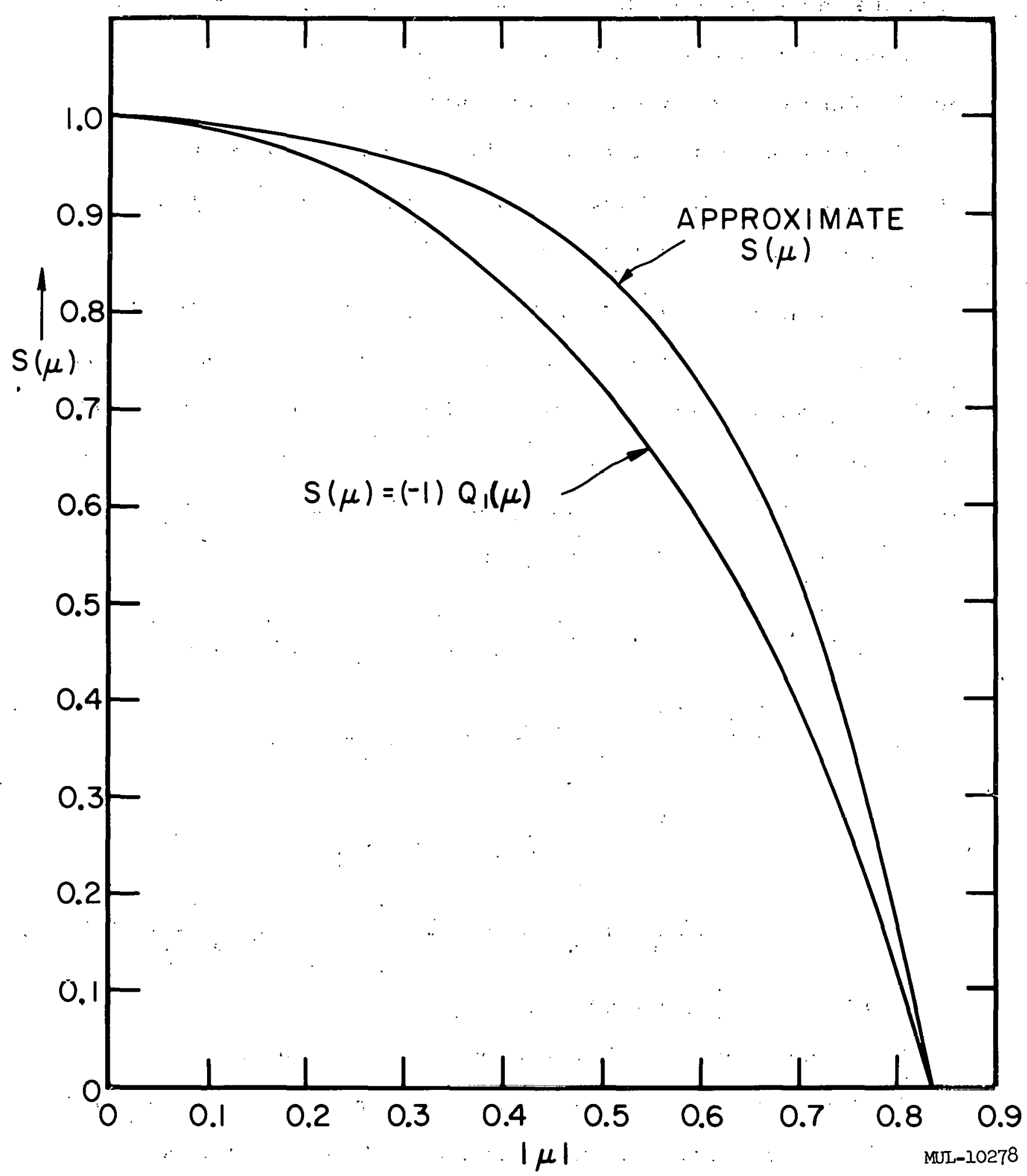

Fig. 2. Comparison of the solution of Eq. (40) for $\lambda=2, S(\mu)=(-1) Q_{1}(\mu)$, with the approximate $S(\mu)$ given by Eq. (42). 
by Eq. (42) with the solution of Eq. (40) for the case $\lambda=2$. The two functions on the average differ by about $9 \%$. For larger mirror ratios. the difference should diminish since for very large $R$ each of the two functions approaches a horizontal straight line at $S(\mu)=1.000$.

Equation (42) was converted to a difference equation and solved numerically with an initial distribution $M_{0}(x)=\exp \left[-10(x-1)^{2}\right]$ and with values of $\lambda$ corresponding to mirror ratios of $3.3,10$, and 100 .

\section{SEPARATED SOLUTIONS WITH A SOURCE FUNCTION}

As mentioned previously some calculations were made with Eq. (30) modified by the addition of a spurce function in order to study the effect of continuously injecting plasma into the mirror machine. These calculations were made only in the separated-solutions approximation. With the addition of a source function Eq. (30) became

$$
\begin{aligned}
\left(\frac{\partial F}{\partial T}\right)_{C} & =4 \pi F^{2}+\frac{1}{2} G_{x x} F_{x x}+\left[\frac{\left(1-\mu^{2}\right)}{x^{2}} G_{\mu x}-\frac{\left(1-\mu^{2}\right)}{x^{3}} G_{\mu}\right] F_{\mu x} \\
& +\left[\frac{\left(1-\mu^{2}\right)^{2}}{2 x^{4}} G_{\mu \mu}-\frac{\mu\left(1-\mu^{2}\right)}{2 x^{4}} G_{\mu}+\frac{\left(1-\mu^{2}\right)}{2 x^{3}} G_{x}\right] F_{\mu \mu} \\
& +\left[\frac{\left(1-\mu^{2}\right)}{2 x^{3}} G_{\mu \mu}-\frac{\mu}{x^{3}} G_{\mu}+\frac{1}{x^{2}} G_{x}\right] F_{x} \\
& +\left[-\frac{\mu\left(1-\mu{ }^{2}\right)}{2 x^{4}} G_{\mu \mu}+\frac{1}{x^{4}} G_{\mu}-\frac{\left(1-\mu^{2}\right)}{3} G_{\mu x}-\frac{\mu}{x^{3}} G_{x}\right] F_{\mu}+W\left(x, \mu, T_{s}\right)
\end{aligned}
$$

where $W\left(x, \mu, \tau_{s}\right)$ represented the sounce function.

Because the initial particle density $n_{0}$ was. assumed to be zero, $\tau_{s}$ could not be related to the time $t$ in the manner of Eq. (35). Consequently $\tau_{s}$ was related to the time by means of the injection current density $J$ through the equations 


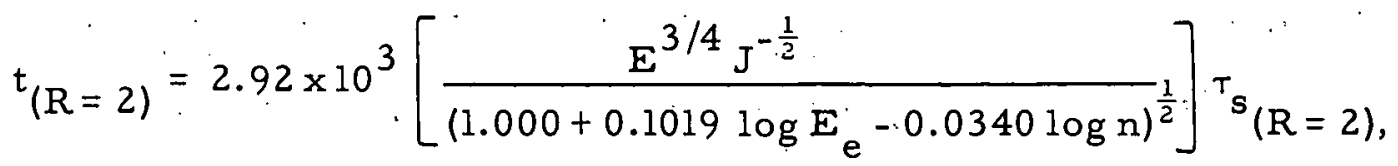

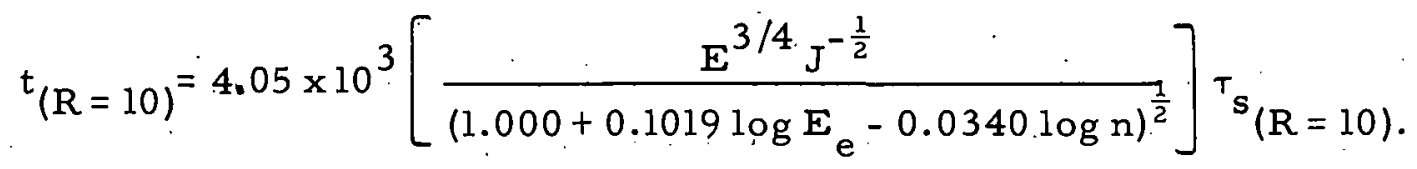

A derivation of Eqs. (49) and (50) is given in Appendix A-3.

To permit separating the variables in $\mathrm{Eq} .(48)$, the source function $W\left(x, \mu, \tau_{s}\right)$ was assumed to have the form $W=K\left(x, \tau_{s}\right) S(\mu)$. As in the separation of variables in. Eq. (30), $G_{\mu}, G_{\mu \mu}$, and $G_{\mu x}$ were set equal to zero, and $F$ was assumed to be equal to $M\left(x ; \tau_{s}\right) S(\mu)$, with $F^{2}=M^{2} S \times l$.

Equation (48) was then separable into the Legendre equation, Eq. (40), and

$$
\left(\frac{\partial M}{\partial \tau_{s}}\right)_{c}=4 \pi M^{2}+\frac{1}{2} G_{x x} M_{x x}+\frac{1}{x^{2}} G_{x} M_{x}-\frac{G_{x}}{2 x^{3}} \lambda M+K\left(x, \tau_{s}\right) .
$$

The source function, $W$, was assumed to be a delta function in $x$, and to be constant in time. Equation (51) was then converted to a difference equation and solved numerically for $\lambda$ 's corresponding to mirror ratios of 2 and 10. The computations were carried out until steady-state distributions were attained,

\section{THE UNSEPARATED SOLUTIONS}

The unseparated solutions were obtained by converting Eq. (30) to a difference equation and numerically solving it. Two different initial distributions were used,

and

$$
F_{0}(x)=e^{-10(x-1)^{2}}\left[(-1) Q_{1}(\mu)\right]
$$

$$
F_{0}(x)=e^{-10(x-1)^{2}} e^{-100 \mu^{2}}
$$

The distribution given by. Eq. (52) is called the normal mode distribution, 
and the distribution given by Eq. (53) is called the peaked-in-angle distribution. Both unseparated solutions were calculated with $\lambda=2$, corresponding to a mirror ratio of 3.3 . Use of the peaked-in-angle initial distribution permitted a study of the effect of injecting the particles into the mirror machine in an average direction more nearly normal to the magnetic field lines than was possible in the separated solutions. The normal mode computation served to verify qualitatively the results of the separated solutions, its chief quantitative effect being to alter the apparent rate of loss of plasma from the mirror ma chine.

\section{NUMERICAL RESULTS OF THE END-LOSS CALCULATIONS:} THE UNSEPARATED SOLUTIONS

Figures $3 \mathrm{~A}$ and $3 \mathrm{~B}$ illustrate the evolution in time of the normal mode unseparated solution. Figure $3 \mathrm{~A}$ is a plot of the distribution function for $\mu=0$ (i.e., at right angles to the magnetic field) versus the relative speed of the particles. Figure $3 \mathrm{~B}$ is a plot of the distribution function at $\mathrm{x}=1$ (representing the speed at the peak of the initial distribution) versus $\mu_{n}$, the cosine of the angle made by the particles with the magnetic field. Figure $3 \mathrm{~B}$ shows the time-evolving distribution function for only positive values of $\mu$, but in all cases the distribution function is symmetric about $\mu=0$. The Maxwell distribution containing the same number of particles as the initial distribution is also plotted in Figs. $3 \mathrm{~A}$ and $3 \mathrm{~B}$ for comparison purposes.

As indicated by Figs. $3 \mathrm{~A}$ and $3 \mathrm{~B}$ and the third column of Table 2, the normal mode distribution loses particles with the passage of time with no essential change in the directional distribution. However, the speed distribution does show some change of shape. The distribution tends to change toward a Maxwellian but is countered by the $1 / v^{4}$ dependence of the 


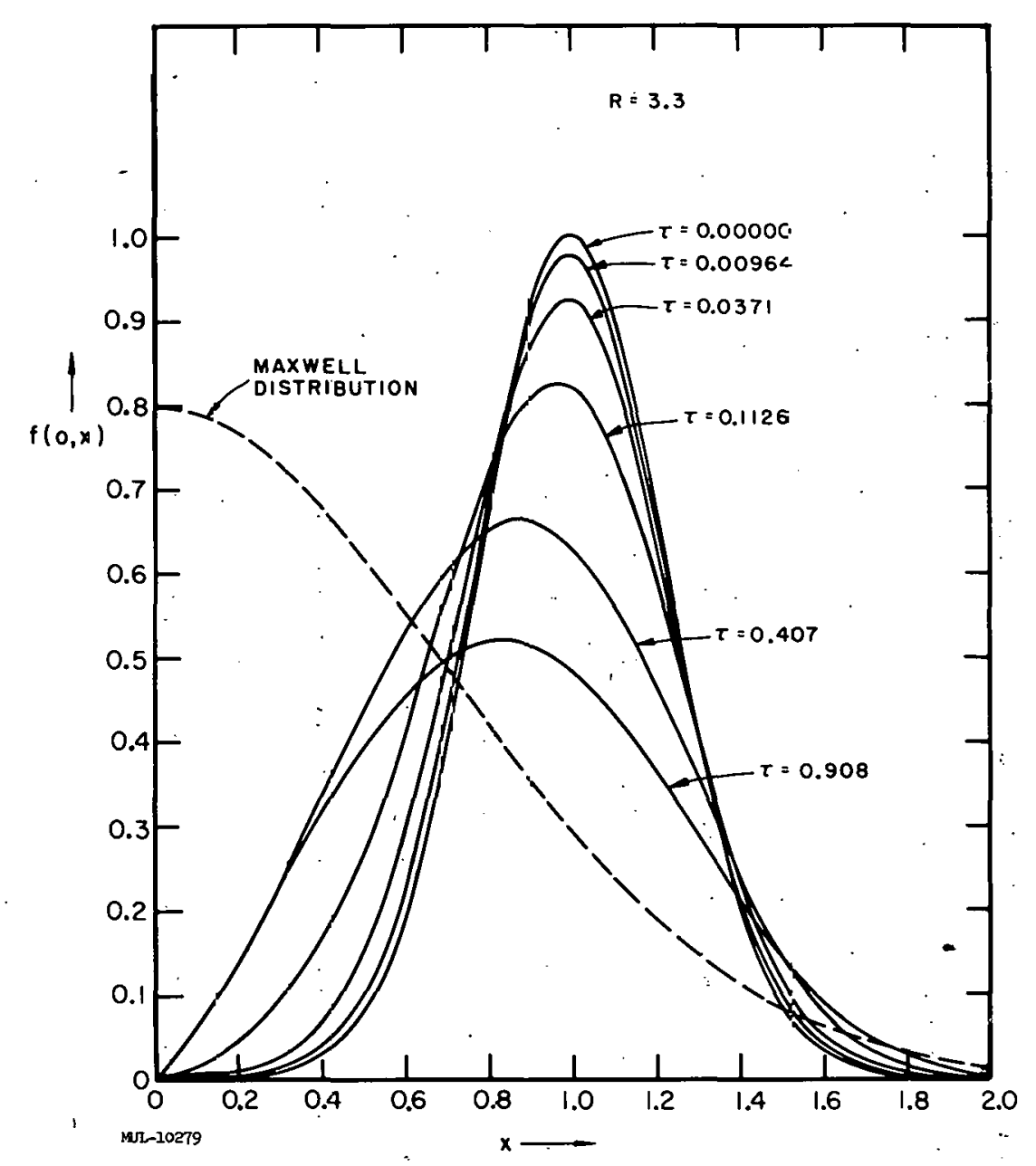

Fig. 3A. Unseparated Solution, $f(0, x)$ vs. $x$, Normal Mode, $R=3.3$.

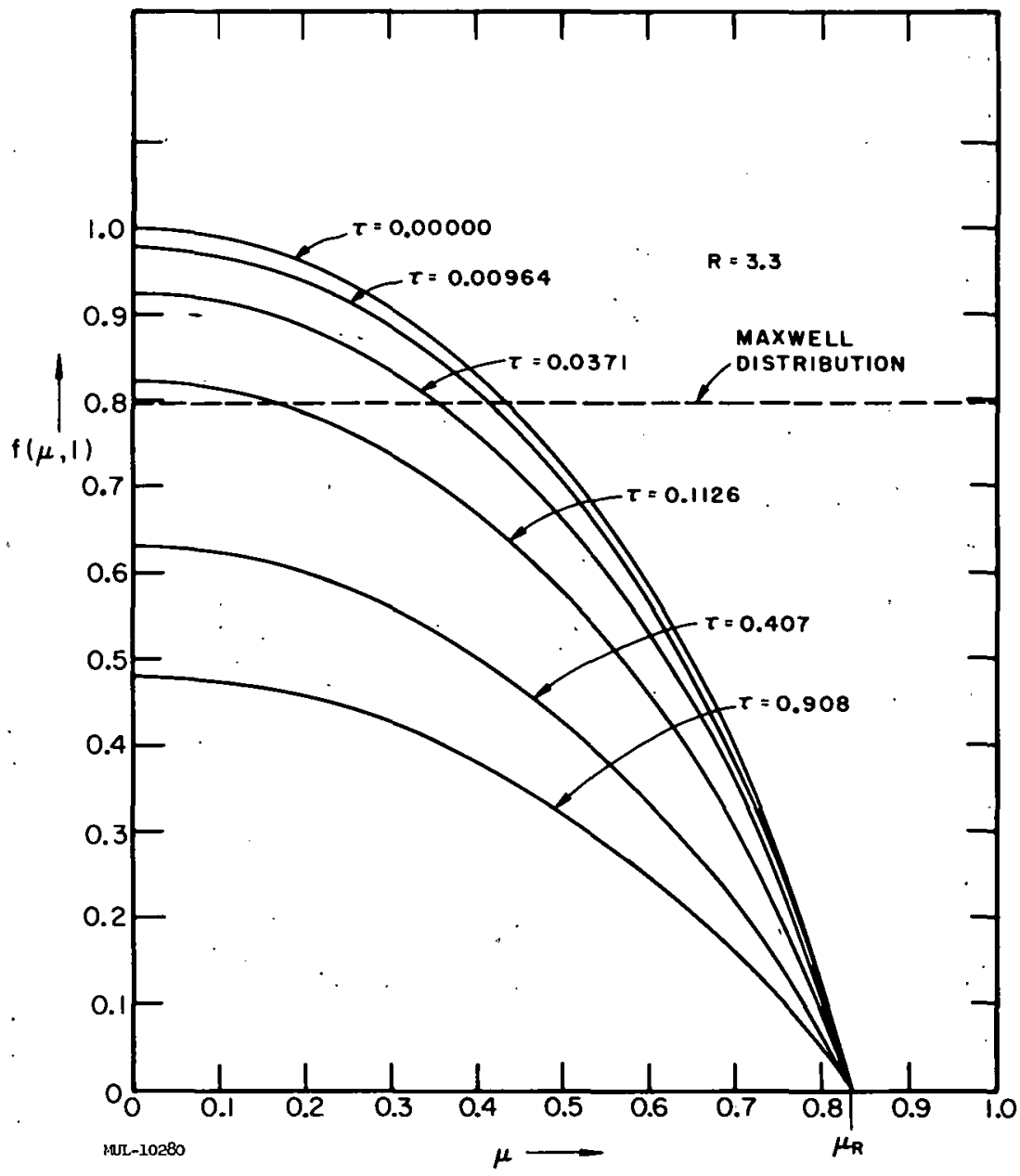

Fig. 3B. Unseparated Solution, $f(\mu, 1)$ vs. $\mu$, Normal Mode, $\mathrm{R}=3.3$. 
TABLE 2. MEAN ENERGY $\overline{x^{2}}$, RELATIVE NUMBER OF PARTICLES Ni: LOSS RATE CONSTANT k, FOR UNSEPARATED NORMAL MODE CALCULATION; $\mathrm{R}=3.3$

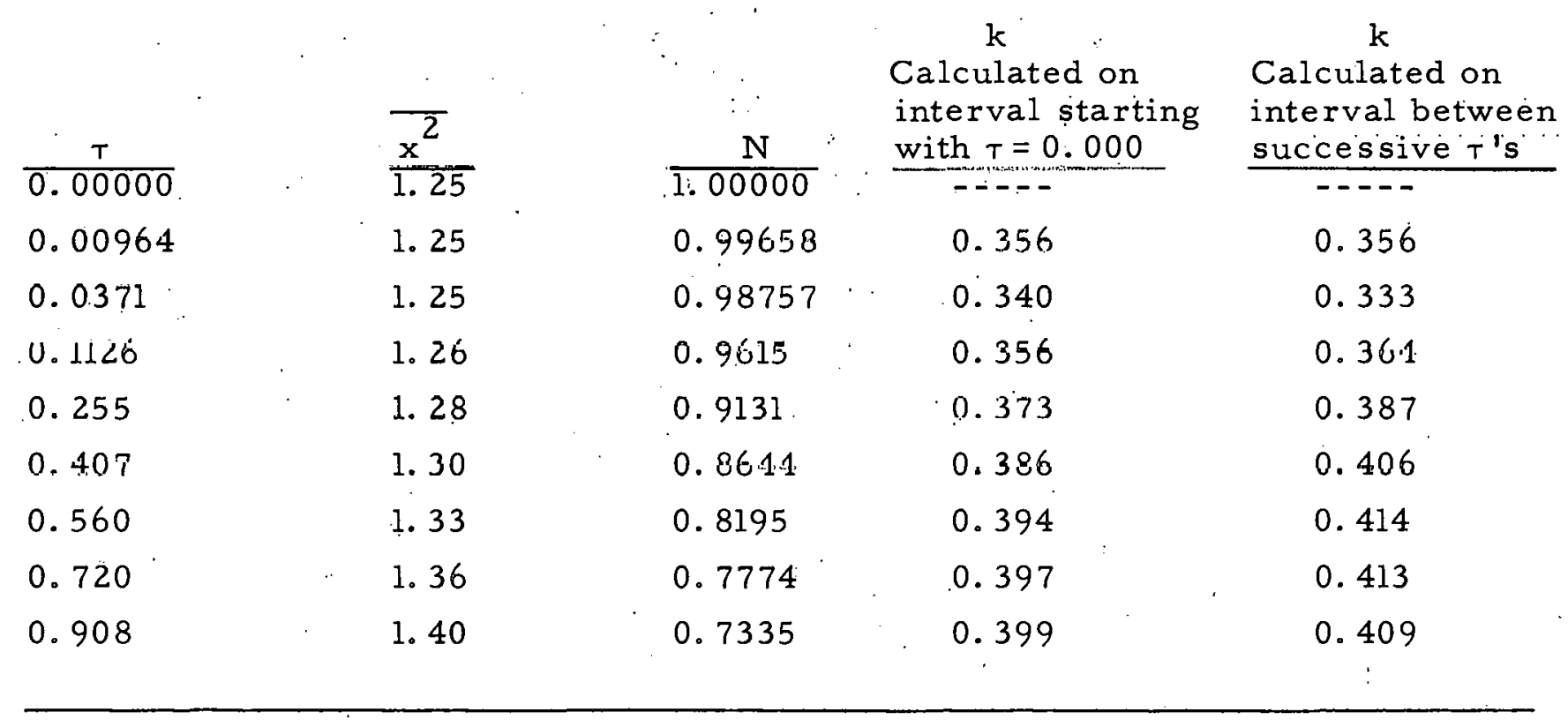

particle scattering cross section. The very low energy particles are almost immediately scattered through large angles and lost from the system through the mirrors. As a consequence the number of low-energy particles is unable to build up to the Maxwellian level. At the high-energy end of the distribution the scattering cross sections are much lower and there is a diminished rate of change of velocity, whether with regard to creating a high-energy Maxwellian tail or to changing directions past the critical angle. As a result the high-energy particles tend to remain in the system while the low-energy ones tend to escape. This effect results in an increase in the average energy of the remaining particles as shown by the values of $\overline{x^{2}}$ given in the second column of Table 2 .

Tabulated in the fourth and fifth columns of Table 2, and plotted in 
Fig. 4, is the apparent particle-los s reaction-rate constant k, calculated on the premise that the particle loss is a second-order reaction, i. e., that

$$
-\frac{\mathrm{dN}}{\mathrm{d} \tau}=\mathrm{kN}^{2}
$$

Taking the extrapolated value of $\mathrm{k}$ from Fig. 4 to be 0.40 , the containment half-life for the normal-mode plasma in a mirror machine of mirror ratio 3.3 is

$$
{ }^{\tau_{1 / 2}(R=3.3)}=\frac{2.5 \times 10^{10}}{\left(1.000+0.1019 \log E_{e^{-}}-0.0340 \log n_{0}\right)} \cdot \frac{E^{3 / 2}}{n_{0}} .
$$

The extrapolation of the curve of Fig. 4 that is used in Eq. (55) is not an extrapolation to infinity. As time passes and themean energy of the remaining particles continues to increase, the Aass rate constant will approach zero. The fifth column of Table 2, plotted with crosses in.Fig. 4, shows that the more nearly instantaneous values of the apparent loss-rate constant are already starting to decrease. For purposes of estimating the time required for the loss of the first $50 \%$ of the initial distribution a value of $k$ of about 0.4 appears to be nearly correct.

Figures $5 \mathrm{~A}$ and $5 \mathrm{~B}$ illustrate the evolution in time of the unseparated peaked-in-angle distribution. Figures $5 \mathrm{~A}$ and $5 \mathrm{~B}$ illustrate the same aspects of the peaked-in-angle distribution as were displayed in Figs. 3A and 3B for the normal mode. There is relatively little change of shape in the speed distribution until the directional dist ribution.spreads out to the critical los angle. On reaching $\mu_{R}$ the directional distribution begins to assume the contour of a normal mode distribution. Simultaneously with the directional distribution reaching $\mu_{R}$ the speed distribution begins to assumethe form approached in the normal mode case with the passage of time. These effects are perhaps better shown by Figs. $6 \mathrm{~A}$ and $6 \mathrm{~B}$ in which some of the later 


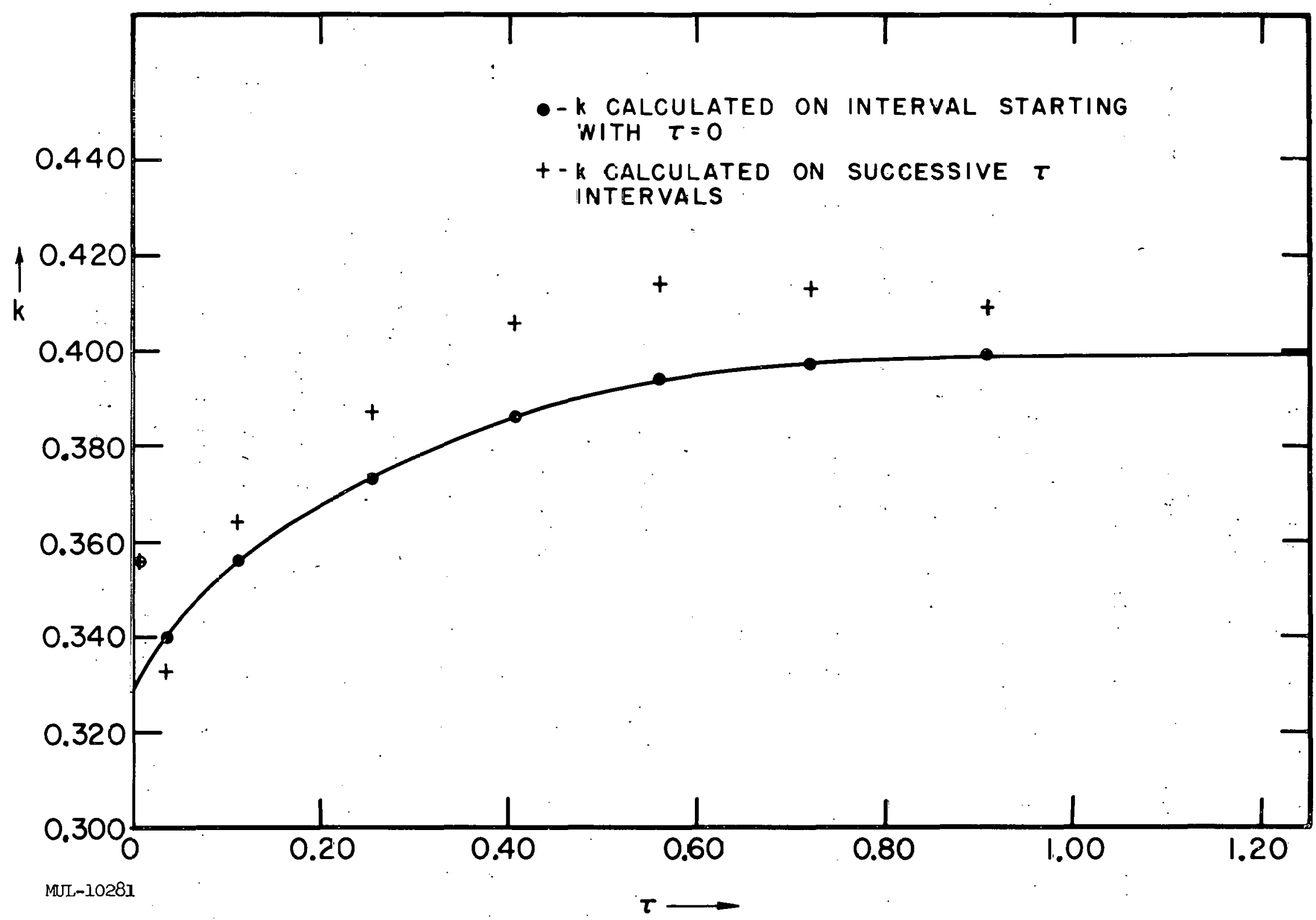

Fig. 4. Apparent End-Loss Reaction Rate Constant k versus $T$, Normal Mode, Unseparated Solution, $R=3.3$. 


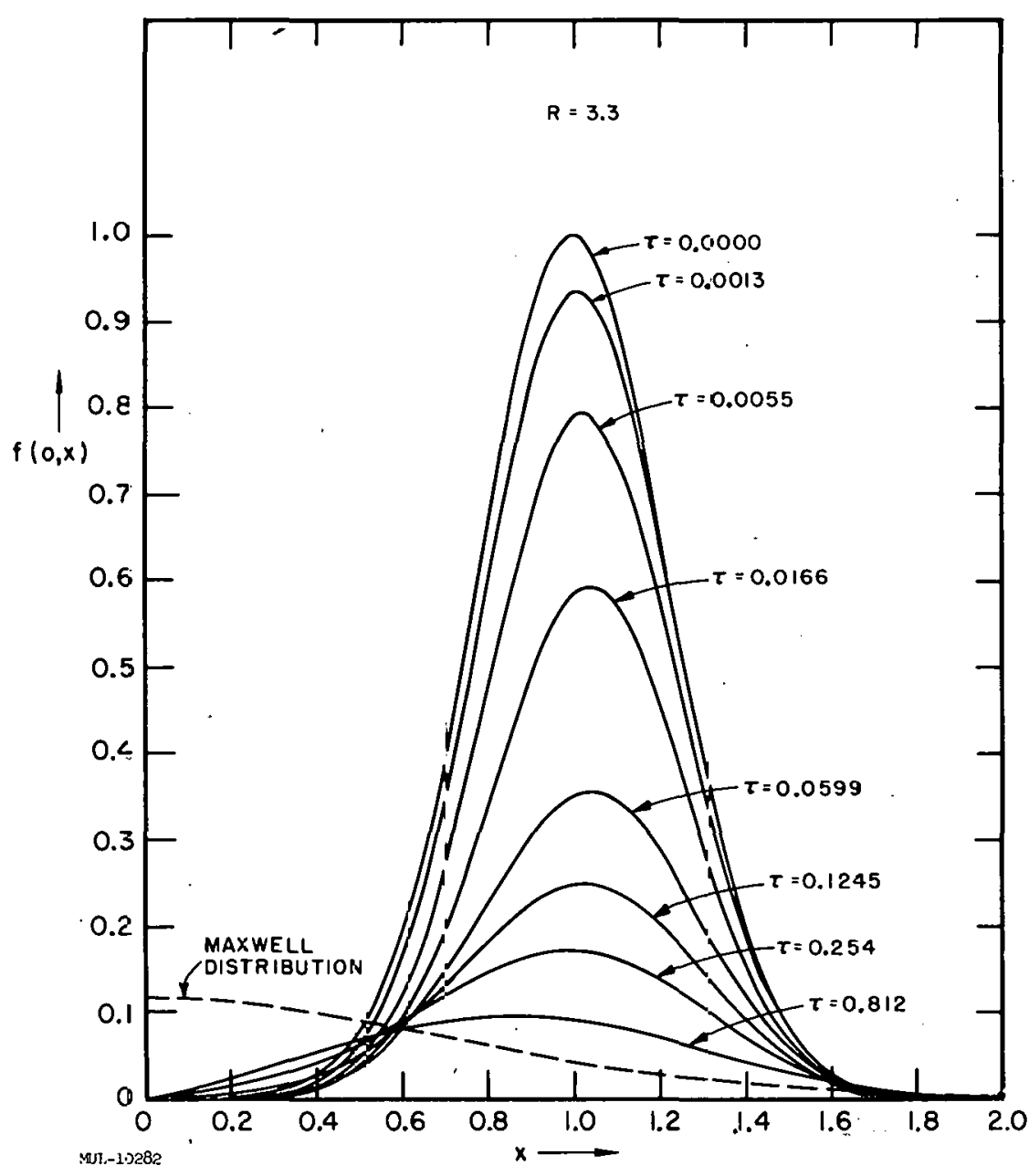

Fig. 5A. Unseparated Solution, $f(0, x)$.vs. $x$, Peaked-in-Angle, $\mathrm{R}=3.3$.

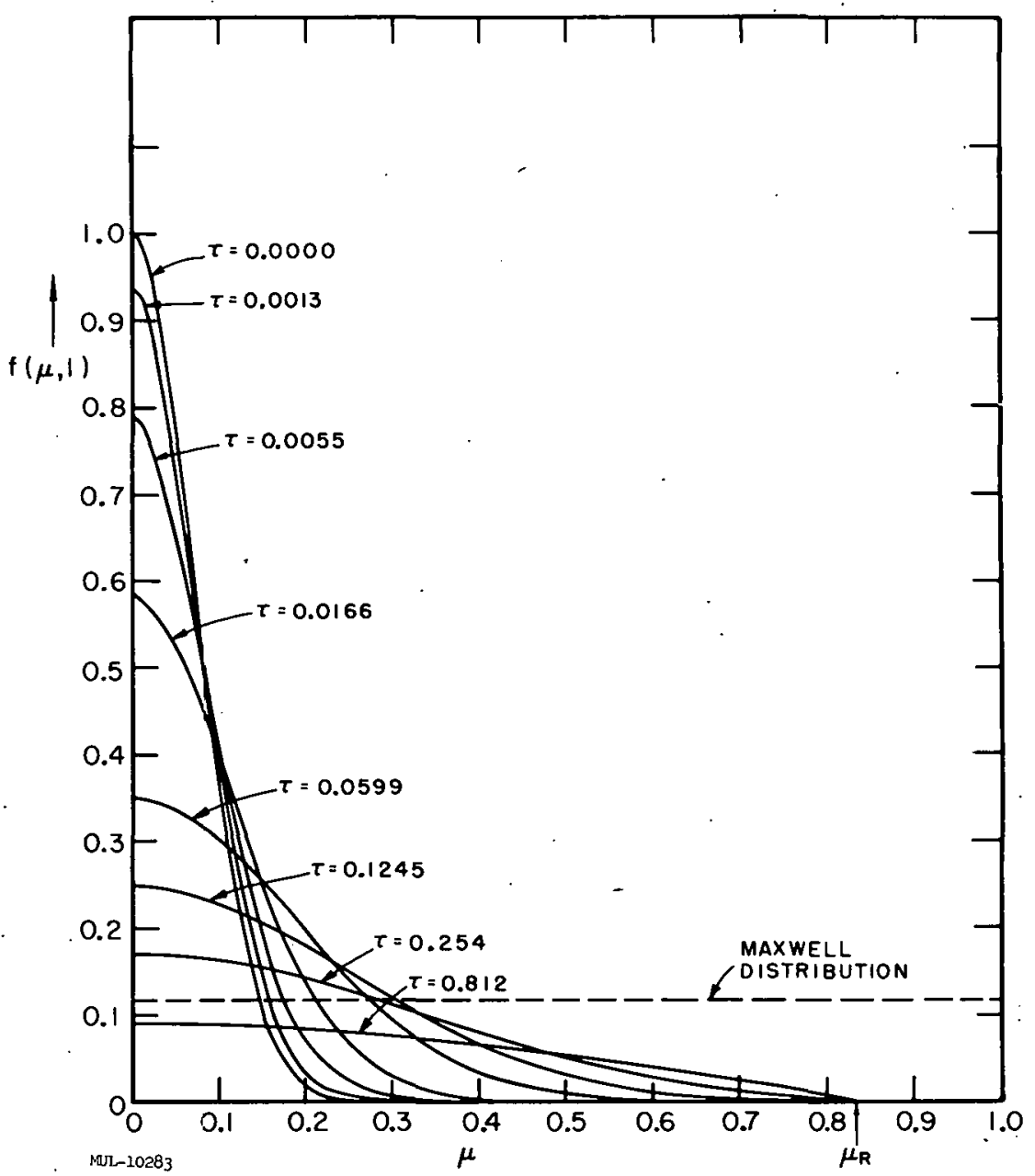

Fig. 5B. Unseparated Solution, $f(\mu, 1)$ vs $\mu$, Peaked-in-Angle, $R=3.3$. 


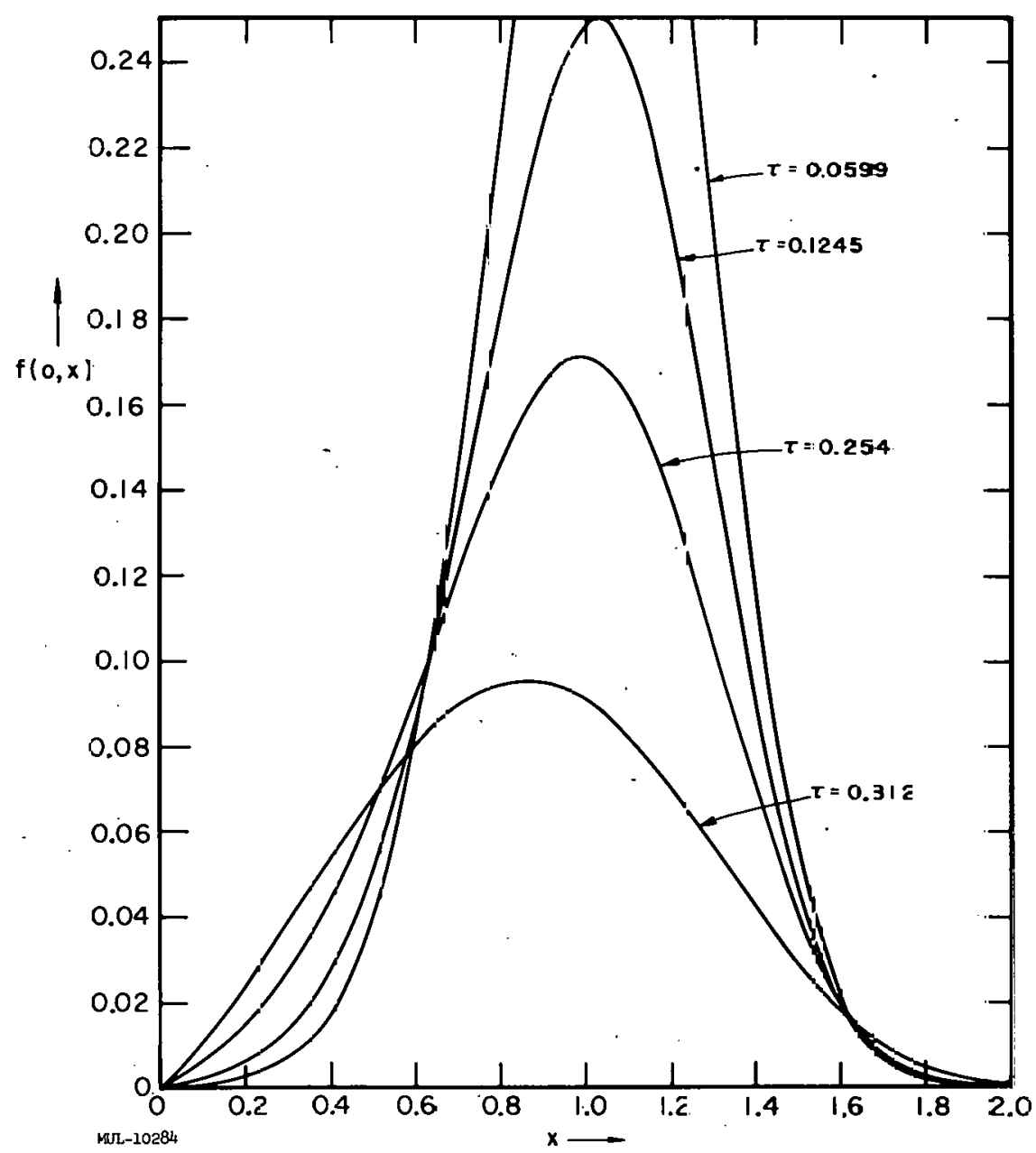

Fig. 6A. Unseparated Solution, $f(0, x)$ vs. $x$,

Peaked-in-Angle, $R=3.3$, Expanded Scale.

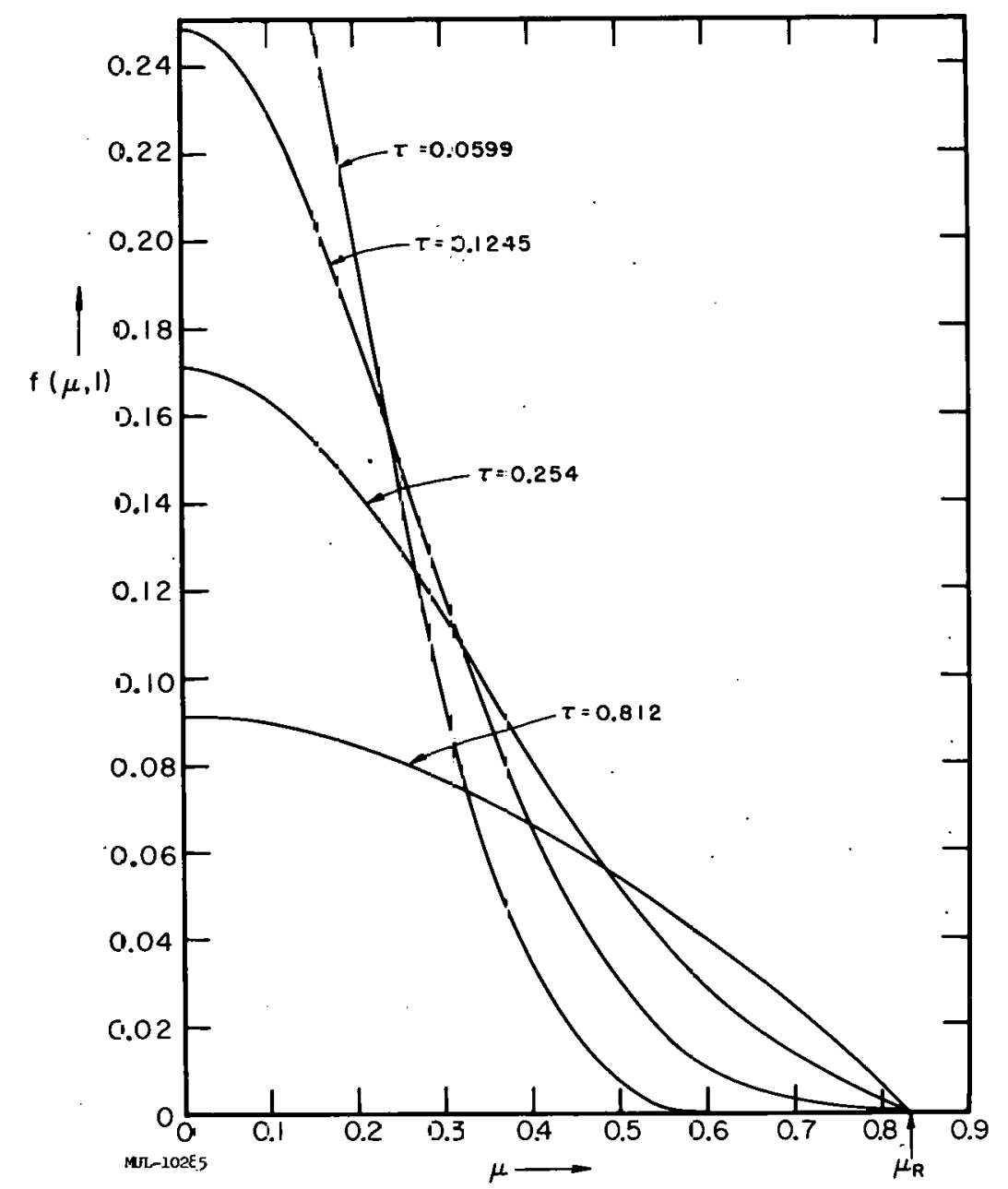

Fig. 6B. Unsefrarated Solution, $f(\mu, 1)$ vs. $\mu$,

Feaked-in-Angle, $R=3.3$, Expanded Scale. 
distributions are plotted on an expanded scale, and by Figs. 7A and 7B in which some normal mode and some peaked-in-angle curves are shown on the same plot. In Figs. $7 \mathrm{~A}$ and $7 \mathrm{~B}$ the curves have been normalized to 1.000 at $x=1, \tau=0$ to facilitate the comparison of their shapes.

In Fig. 8 the relative number of particles is plotted against $\tau$ for the peaked-in-angle distribution. The relative number of particles is seen to drop initially at an extremely rapid rate to a plateau at about 0.889 , from which a steadily increasing rate of loss sets in at about $\tau=0.124$. As shown in Figs. 5B, 6B, and 7B, the directional distribution begins to as sume nonzero values near $\mu_{R}$ between $\tau=0.1245$ and $\tau=0.254$ consistent with the onset of particle loss.

The initial sharp decrease in the relative number of particles apparently results from truncation errors made by the computing machine in calculating the various derivatives of Eq. (53). The machine evaluated $F$ at only ten equally spaced values of $\mu$ (including $\mu=0$ and $\mu=\mu_{R}$ ), which makes it seem reasonable that the present computation was performed on too coarse a scale for the initial peaked-in-angle distribution. A particle verification of this explanation was obtained when the derivatives $F_{x^{\prime}} F_{\mu}, F_{x x^{\prime}}, F_{\mu x^{\prime}}$ and $F_{\mu \mu}$ were obtained analytically from the initial distribution function and were used in computing the first time step. This resulted in the elimination of more than half of the apparent particle loss obtained when the first time step was completely machine-computed. It is also likely that the 0.1 interval of $x$ values used by the machine was slightly too coarse for the initial distribution function. This could account for the slightly higher initial. rate of loss in the normal mode dịtribution as illustrated by the off-the-curve point of Fig. 4. The truncation errors seemed to disappear with time as the peaked-in-angle distribution spread out and its derivatives were more accurately calculated by the machine. 


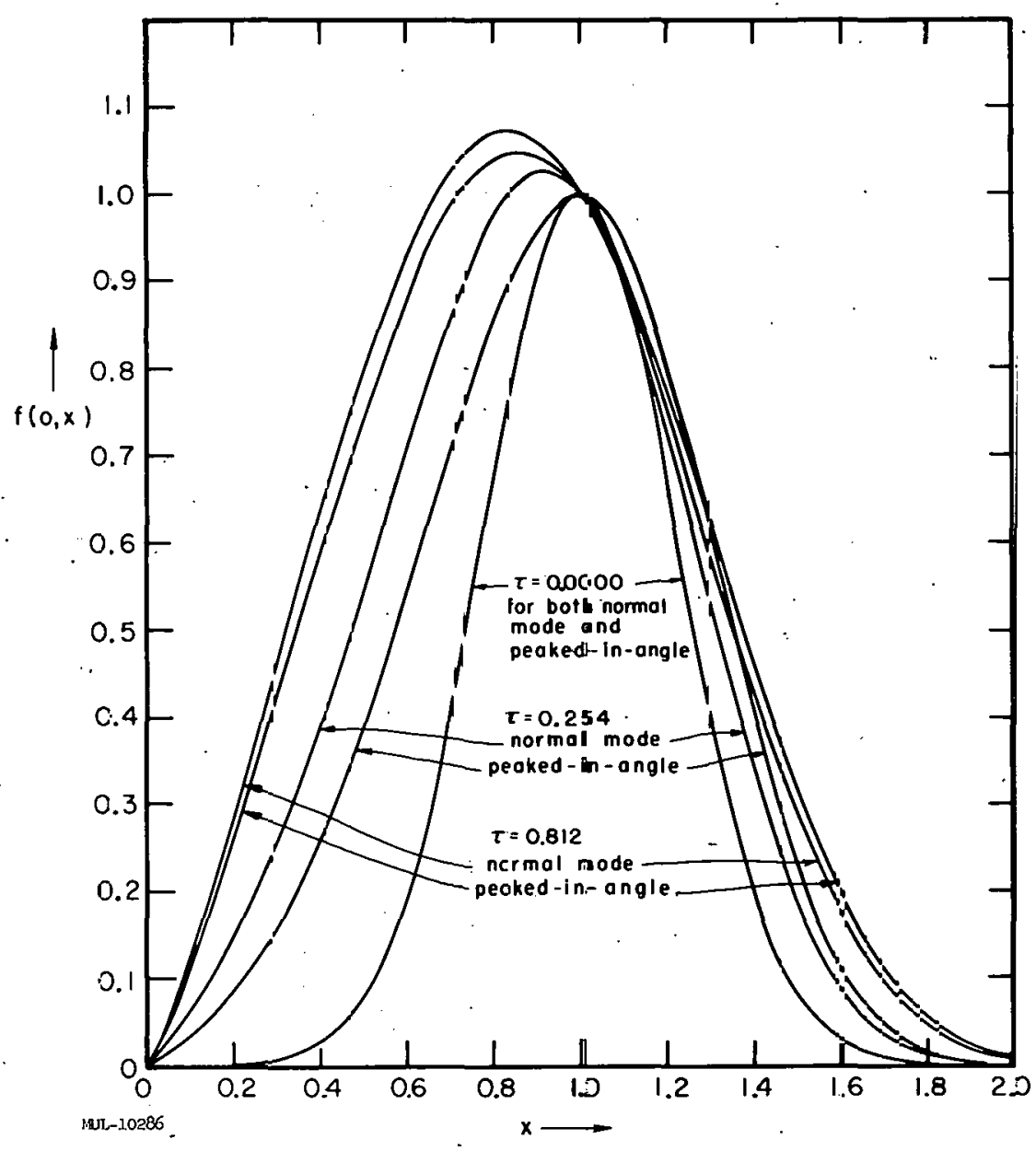

Fig. 7.4. Comparison of some normal mode and peaked-in-angle distributions, unseparated solutions, $R=3.3$. All curves are normalized to 1.000 at $x=1$.

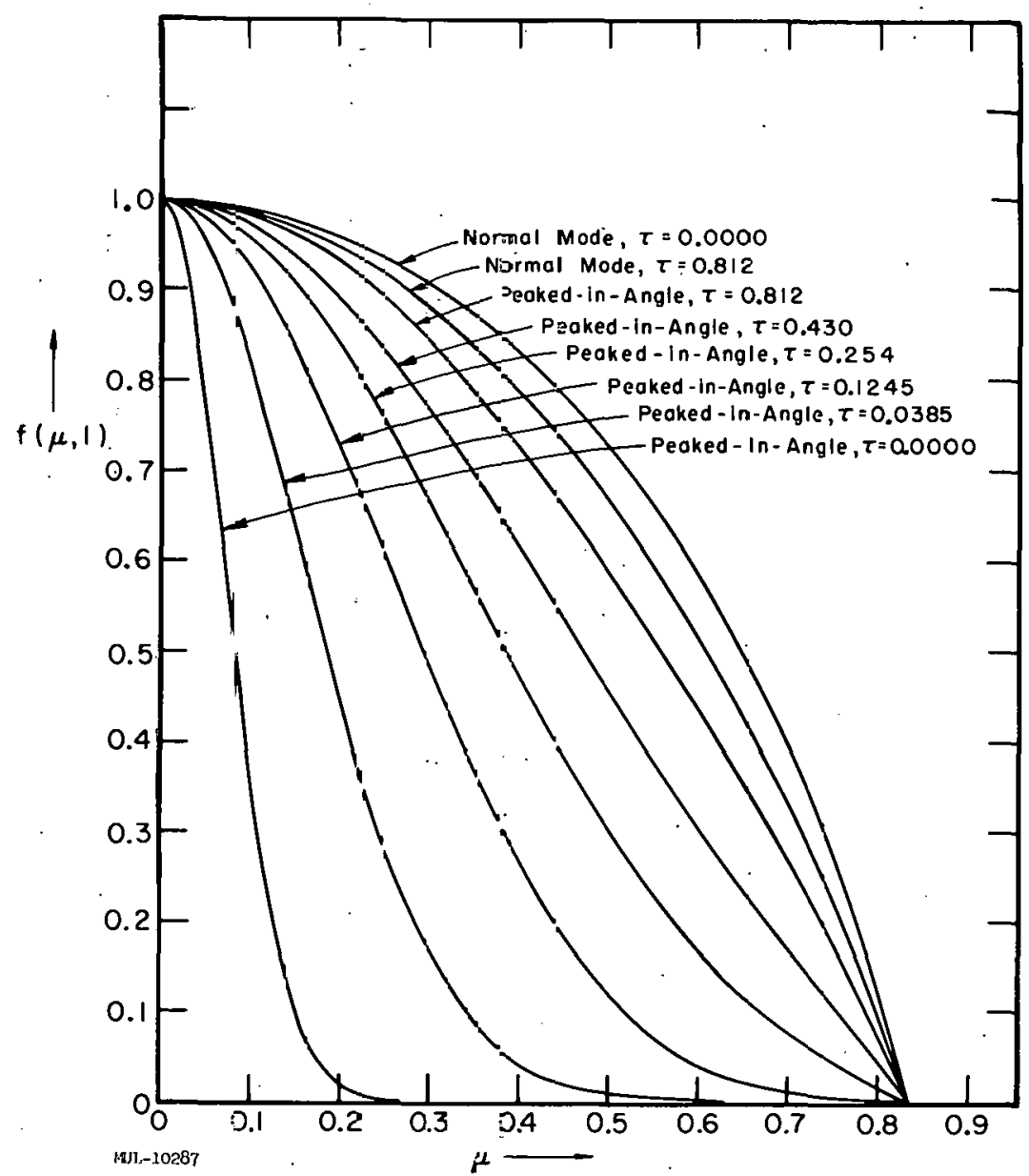

Fig. 7B. Comparison of some normal mode and peaked-in-angle distributions, unseparated solutions, $R=3.3$. All curves are normalized to 1.000 at $\mu=1$. 
UCRL 5651-T

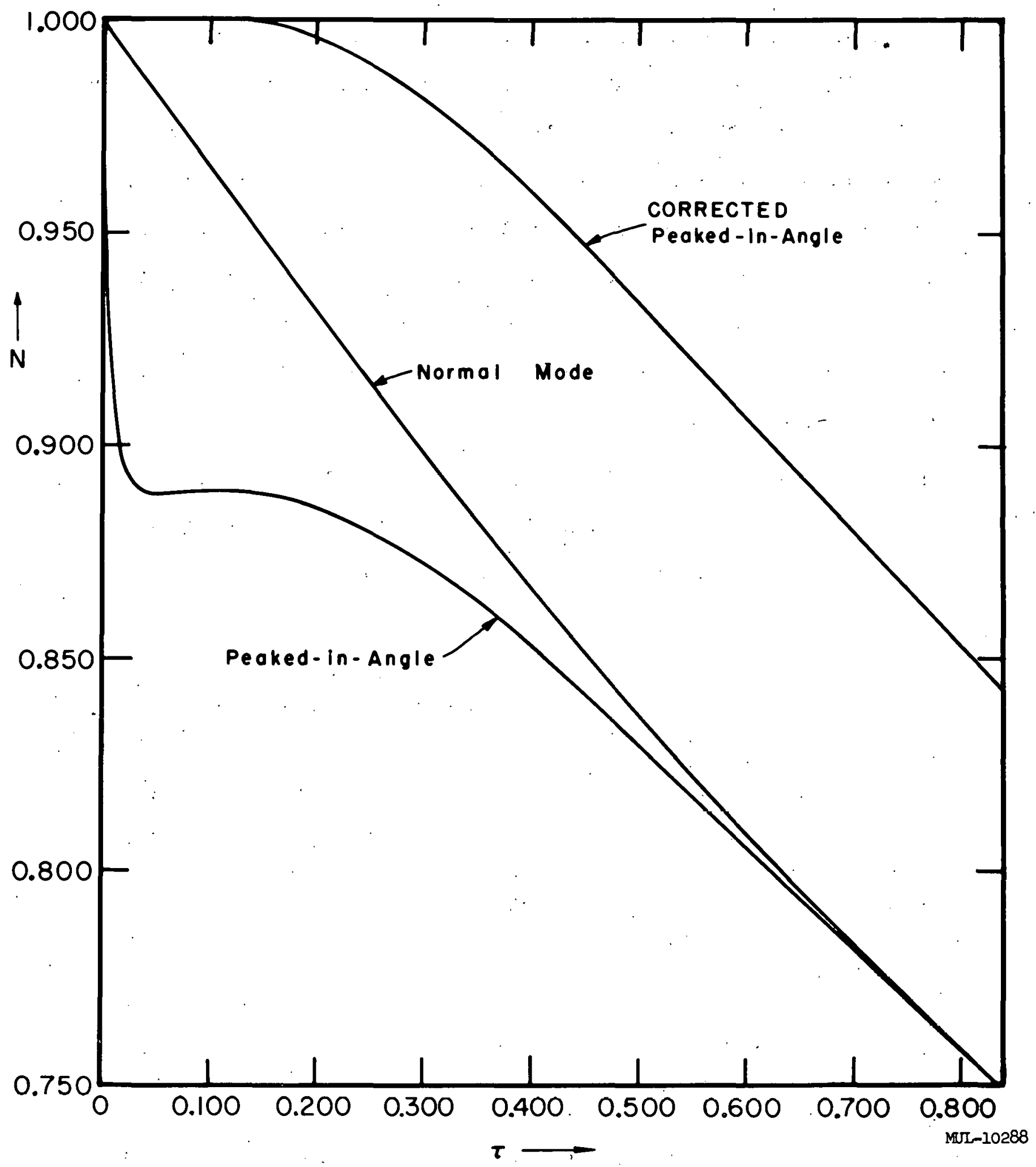

Fig. 8. Plot of $\mathbf{N}$ versus $T$ for Unseparated Solution, $R=3.3$. 
Since the initial drop in the number of particles in the peaked-in-angle case sęemed to be due to computational inaccuracies, the peaked-in-angle curve of Fig. 8 was corrected by ignoring the initial loss and normalizing the other values of $\mathrm{N}$ by giving the plateau the value 1.000. The values of $\mathrm{N}$ appearing in Table 3 are corrected values. The corresponding $\mathrm{k}$ values were computed from the corrected $N$ values on the assumption that the particle loss is a second-order reaction. Inspection of the $k$ values reveals that they are increasing as the distribution approaches the form of the normal mode. Presumably, after sufficient time has passed, the loss-rate constants for the peaked-in-angle and normal mode distributions.will become nearly identical.

TABLE 3. MEAN ENERGY $\overline{x^{2}}$, RELATIVE NUMBER OF PARTICLES N, APPARENT LOSS RATE CONSTANT k, FOR (UNSEPARATED) PEAKED-IN-ANGLE CALCULATIONS, $R=3.3$

\begin{tabular}{|c|c|c|c|c|c|}
\hline$\tau$ & $x^{2}$ & $\mathrm{~N}$ & $\begin{array}{l}\text { k. } \\
\text { Calculated on } \\
\text { intervalstarting } \\
\text { with } \tau=0.0000 \\
\end{array}$ & $\begin{array}{l}\quad k \\
\text { Calculated on } \\
\text { interval starting } \\
\text { with } \tau=0.400 \\
\end{array}$ & \begin{tabular}{l}
\multicolumn{1}{c}{$\mathrm{k}$} \\
Calculated \\
on interval \\
between \\
successive $\mathrm{T}$ s.
\end{tabular} \\
\hline 0.0000 & 1. 25 & 1.0000 & ----- & ---- & $\cdots--$ \\
\hline 0.0013 & 1. 25 & 1.0000 & 0.000 & ---- & 0.000 \\
\hline 0.0055 & 1.26 & 1.0000 & 0.000 & $-\cdots$ & 0.000 \\
\hline 0.0166 & 1.25 & 1.0000 & ก. $\cap \cap \cap$ & $=---$ & 0.000 \\
\hline 0.0599 & 1.25 & 1.0000 & 0.000 & $-\cdots-$ & 0.000 \\
\hline 0.1245 & 1.25 & 1.0000 & 0.000 & $\ldots-\ldots$ & 0.000 \\
\hline 0.254 & 1. 26 & 0.9893 & 0.043 & $-\ldots$ & 0.084 \\
\hline 0.400 & 1. 28 & 0.9596 & 0.105 & $\ldots$ & 0.214 \\
\hline 0.480 & 1.29 & 0.9396 & 0.134 & 0.267 & 0.267 \\
\hline 0.560 & 1.31 & 0.9180 & 0.160 & 0.295 & 0.313 \\
\hline 0.640 & 1.33 & 0.8963 & 0.181 & 0.306 & 0.330 \\
\hline 0.720 & 1. 34 & 0.8746 & 0.199 & 0.316 & 0.346 \\
\hline 0.800 & 1.36 & 0.8534 & 0.215 & 0.324 & 0.355 \\
\hline 0.812 & 1.36 & 0.8503 & 0.217 & 0.325 & \\
\hline
\end{tabular}


However, inspection of Fig. 8 (with necessary extrapolations) indicates that by peaking the initial distribution? one gains a nearly constant additional a mount of time for the containment of any given fraction of the plasma, at least through the relatively early stages of the particle loss. Thus, in comparison with the normal mode, peaking the initial distribution doubles the time required for a $10 \%$ loss of the initial plasma, adds about $50 \%$ to the time required for $20 \%$ los s of the initial plasma, etc.

\section{NUMERICAL RESULTS OF THE END-LOSS CALCULATIONS: THE SEPARATED SOLUTIONS ${ }^{16}$}

The unseparated solutions illustrate the effects of varying the directional distribution function at constant mirror ratio. As mentioned earlier, the unseparated solutions were also more accurate than the separated solutions. However, the unseparated and separated solutions were in qualitative agreement and the latter were useful for illustrating, the effects on the normal. mode distribution caused by varying the mirror ratio.

s The separated solution distribution functions for the mirror ratios 3.3 , 10, and 100 are plotted in Figs. $9 \mathrm{~A}$ and $9 \mathrm{~B}, 10 \mathrm{~A}$ and $10 \mathrm{~B}$, and $11 \mathrm{~A}$ and $11 \mathrm{~B}$. The number of particles as a function of $\tau$ for each of those mirror ratios is plotted in Fig. 12. Figures 9, 10, 11, and 12 show a diminishing rate of loss of plasma with increasing mirror ratio. Figures 9, 10 and 11 also show that as the mirror ratio is increased there is an increased build-up of both the low- and high-energy regions of the distribution function with the passage of time.

${ }^{16}$ This section is based upon numerical results obtained by M. N. Rosenbluth, W. M. MacDonald, and W. Chuck. 


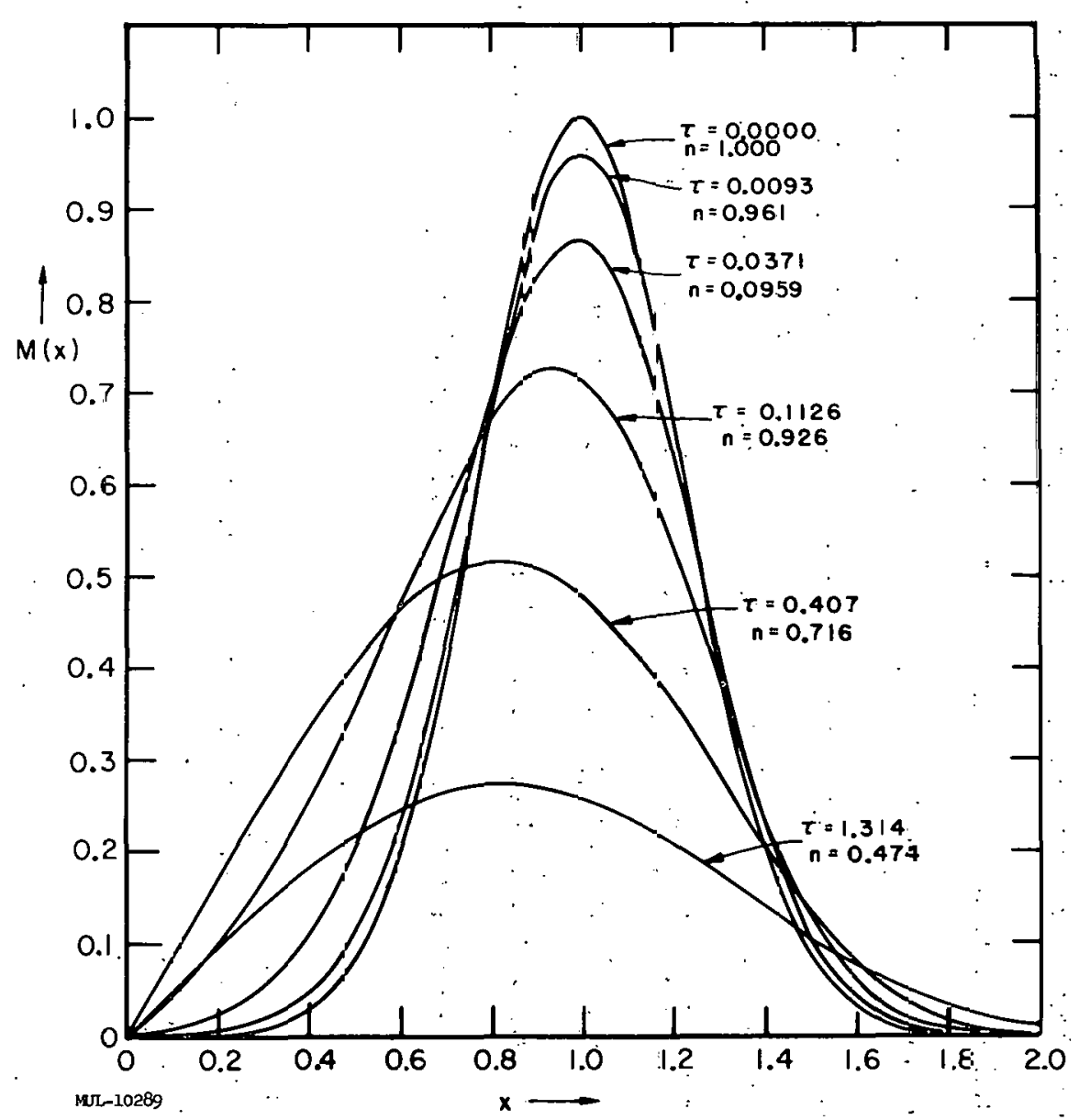

Fig. 9A. Separated Solutions, $M(x)$ vs. $x$, $\mathrm{R}=3.3$.

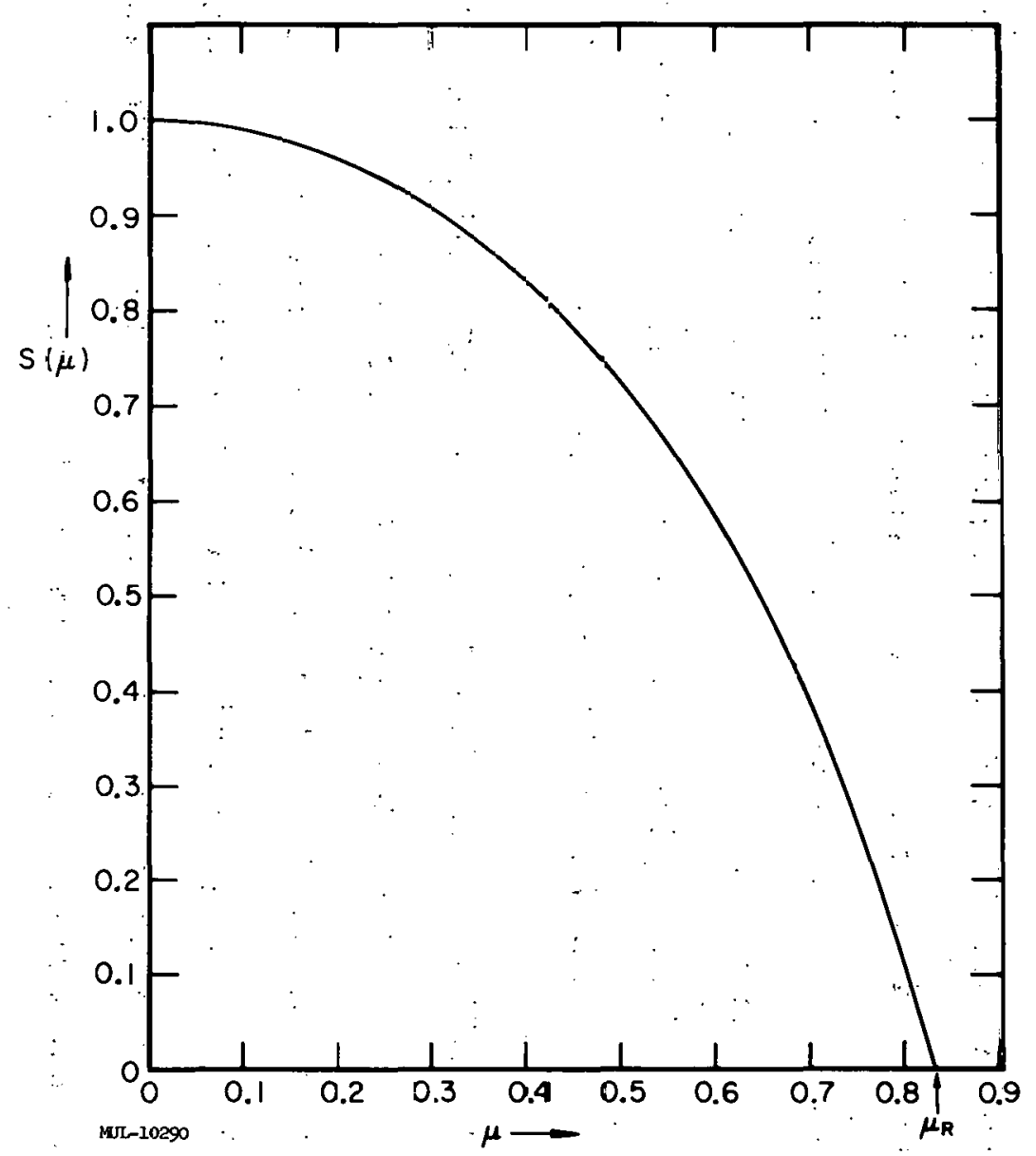

Fig. 9B. Separated Solution, $S(\mu)$ vs. $\mu$, $R=3 . \Im(S(\mu)$ is time independent. ) 


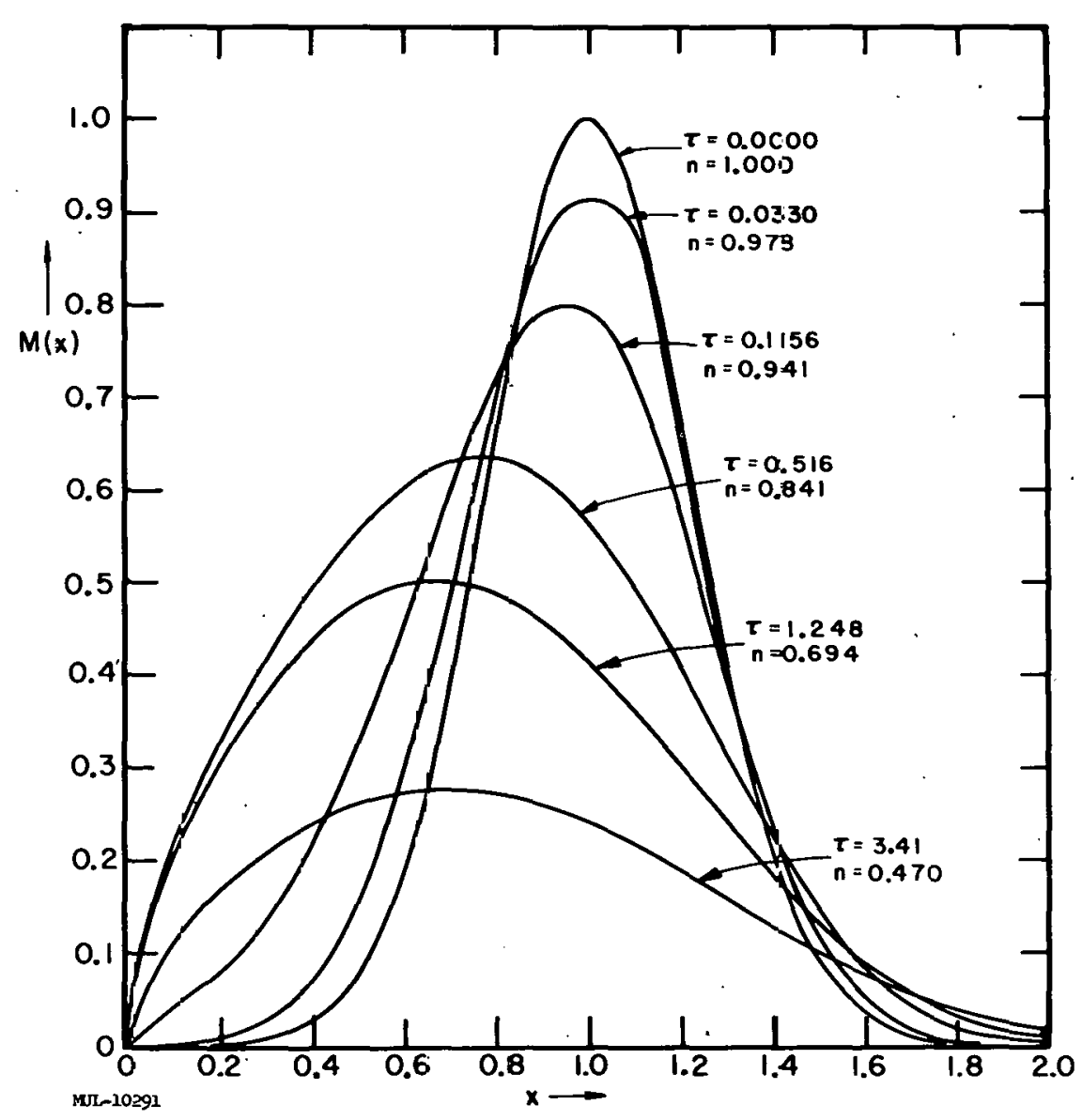

Fig. 10A. Separated Solution, $M(x)$ vs. $x$, $\mathbf{R}=10$.

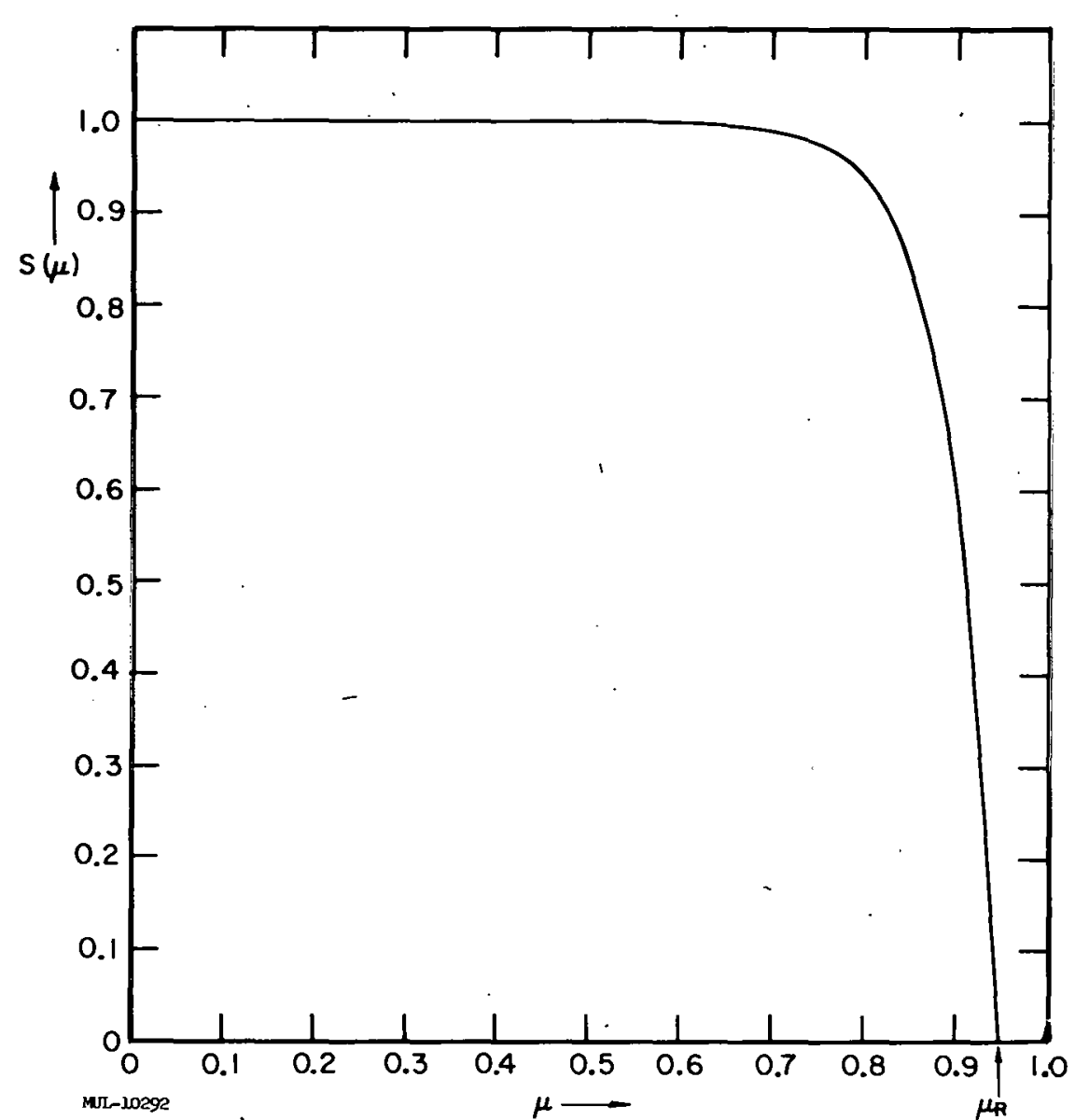

Fig. 10B. Separated Solution, $S(\mu)$ vs. $\mu$, $R=10 . \quad(S(\mu)$ is time independent. $)$ 


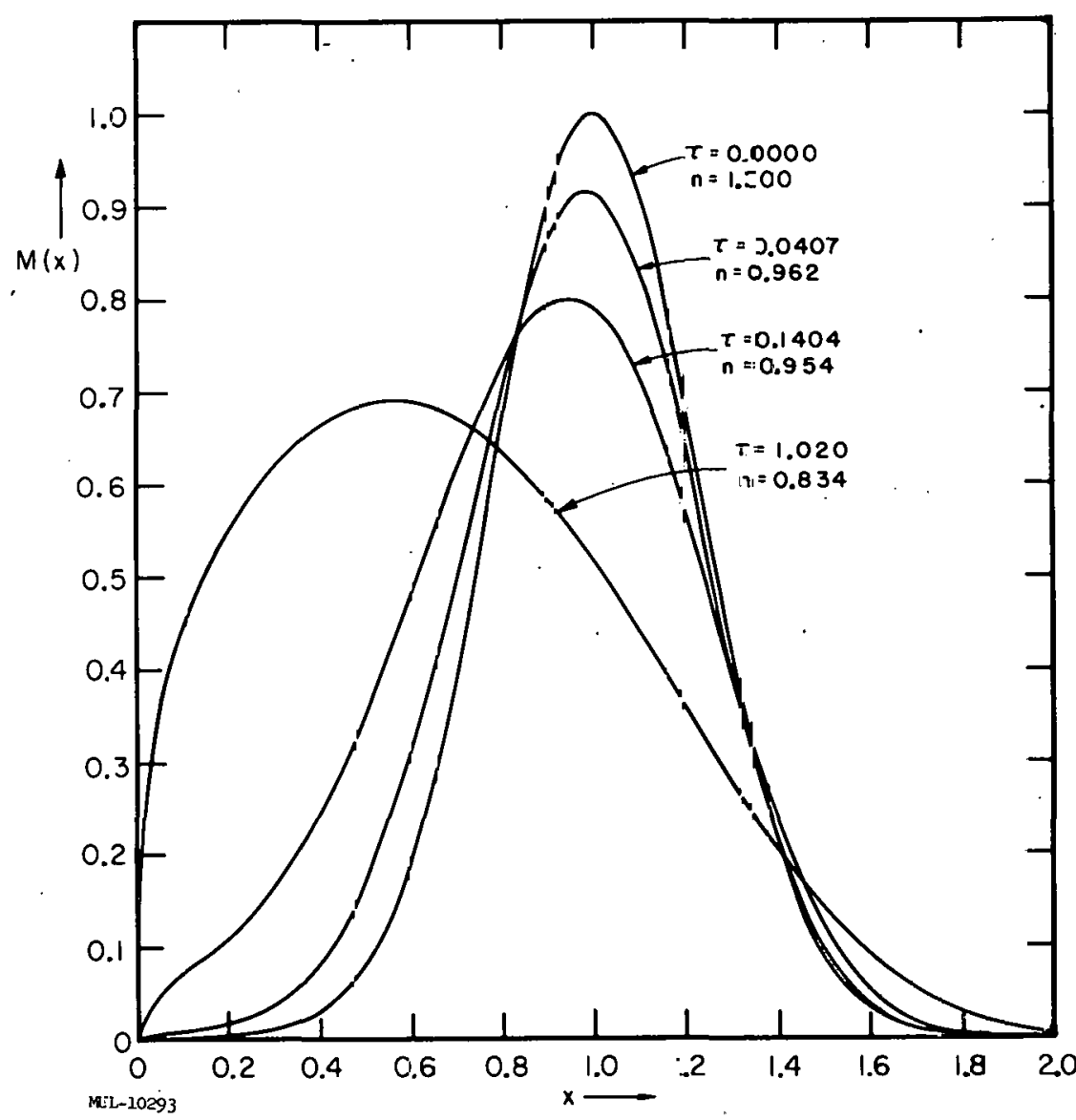

Fig. Il A. Separated Solution, $M(x)$ vs. $x$, $\mathrm{R}=100$.

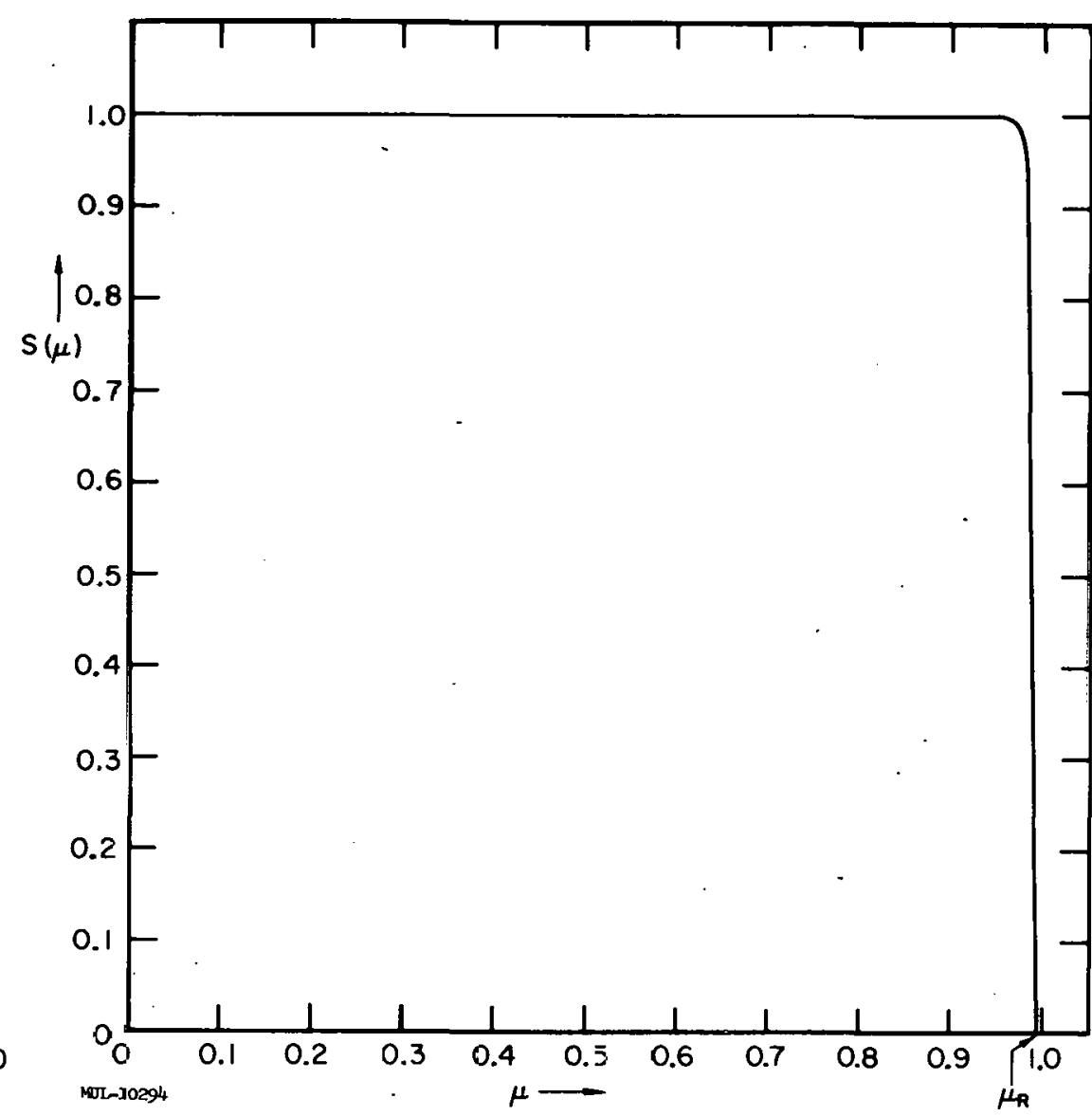

Fig. 11B. Separated Solution, $S(\mu)$ vs. $\mu$, $R=100 . \quad(S(\mu)$ is time independent. $)$ 


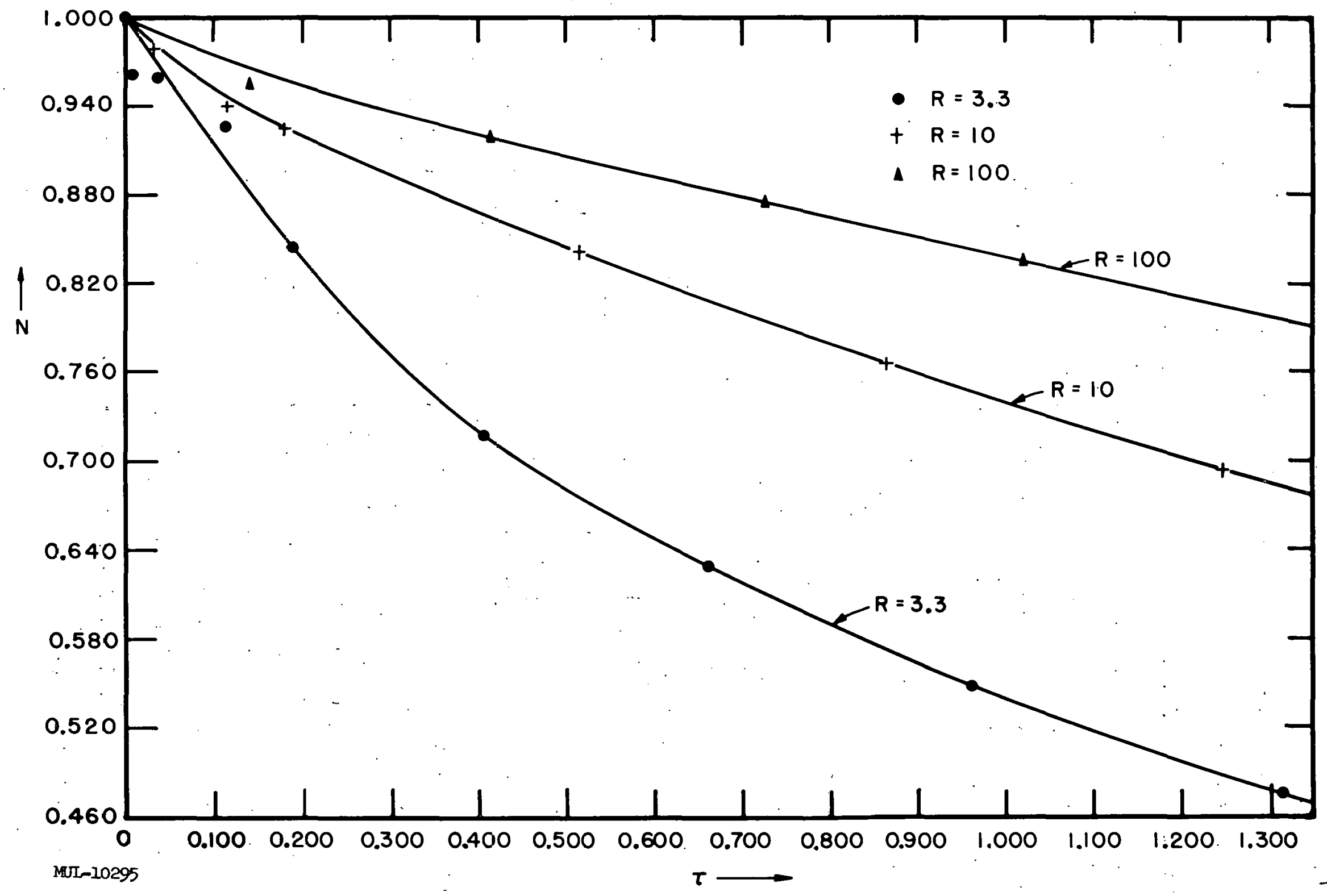

Fig. 12. Relative Number of Particles versus $T$ for the Separated Solutions. 
Relative particle densities at various $\tau^{\prime}$ 's and some values of the apparent loss-rate constant $\mathrm{k}$ are tabulated in Table 4 for the different mirror ratios. A comparison of the $k$ 's of Table 4 (for $R=3.3$ ) with those of Table 2 would seem to indicate that the separated solution gave a rate of loss about twice that given by the unseparated solution. The k's of Table 4 all seem to be too high for small $T$ 's, which is probably consistent with the scattered points at small $\tau$ in Fig. 17. These results seem to indicatc that there were lruntualiun or other errors at small $T$ in the separated calculations.

TABLE 4. TABULATION OF N AND k VALUES FOR THE SEPARATED SOLUTIONS

\begin{tabular}{|c|c|c|c|c|c|c|c|c|}
\hline \multicolumn{3}{|c|}{$R=3.3$} & \multicolumn{3}{|c|}{$R=10$} & \multicolumn{3}{|c|}{$R=100$} \\
\hline$T$ & $\mathrm{~N}$ & $\begin{array}{l}\quad k \\
\text { (Calc'd } \\
\text { from } \\
\text { suc- } \\
\text { cessive } \\
\text { T entries }\end{array}$ &.$T$ & $\mathrm{~N}$ & $\begin{array}{l}\quad k \\
\text { (Calc'd } \\
\text { from } \\
\text { suc- } \\
\text { cessive } \\
\text { T entries) }\end{array}$ & $\tau$ & $\mathrm{N}$ & $\begin{array}{l}\quad k \\
\text { Calc'd } \\
\text { from } \\
\text { suc- } \\
\text { cessive } \\
\text { T entities) }\end{array}$ \\
\hline 0.0000 & 1.000 & & 0.0000 & 1.000 & & 0.0000 & 1.000 & \\
\hline 0.0093 & 0.961 & & 0.0330 & 0.978 & & 0.0407 & 0.962 & \\
\hline 0.0371 & 0.959 & & 0.1156 & 0.941 & 0.486 & 0.1404 & 0.954 & 0.197 \\
\hline 0.1126 & 0.926 & & 0.1806 & 0.925 & 0.283 & 0.413 & 0.918 & $0: 151$ \\
\hline 0.1909 & 0.844 & & 0.516 & 0.841 & 0.323 & ก. 726 & 0.876 & 0.170 \\
\hline 0.407 & 0.716 & 0.981 & 0.864 & 0.766 & 0.334 & 1.020 & 0.834 & 0.196 \\
\hline 0.661 & 0.629 & 0.761 & 1. 248 & 0.694 & 0.353 & & & v \\
\hline 0.962 & 0.547 & 0.792 & 3.41 & 0.470 & 0.318 & & & \\
\hline 1.314 & 0.474 & 0.800 & & & & & & \\
\hline
\end{tabular}

On the assumption that the k's of Table 4 are internally. consistent, it is interesting to compare $\mathrm{k}^{\prime} \mathrm{s}$ at the same $\mathrm{T}$ for the various mirror ratios. Interpolating in the $R=3.3$ and $R=10$ columns to compare with $R=100$ at $\tau=1.020$, one obtains the comparison 


$$
\mathrm{k}_{3.3}: \mathrm{k}_{10}: \mathrm{k}_{100}:: 49-05: 1.74: 1.00
$$

UCRL 5651-T

which suggests that $k \sim \frac{1}{\log R}$ over the above range of $R$ because $k \propto \frac{1}{\log R}$ would give

$$
k_{3.3}: k_{10}: k_{100}:: 4: 2: 1:
$$

Again interpolating in Table 4, one sees that the time required for $\mathrm{N}$ to decay to 0.474 when $R=10$ is 2.56 times as long as the time required when $\mathrm{R}=3.3$. The number 2.56 is to be compared with the reciprocal of the ratio of the $\mathrm{k}^{\prime} \mathrm{s}$ discussed above, which is. 2.33 .

. XI. NUMERICAL RESULTS OF THE END-LOSS CALCULATIONS: THE SEPARATED SOLUTIONS WITH A SOURCE FUNCTION ${ }^{17}$

The steady-state distributions in speed for $R=2$ and $R=10$, resulting from a constant-in-time $\delta$-function source at $x=1$, a re shown in Fig. 13 . The effect on the distribution of varying the mirror ratio is quite apparent. In addition to the over-all magnitude of the distribution function being greater at $\mathrm{R}=10$ than at. $\mathrm{R}=2$, at the larger mirror ratio there is a much greater relative amount of both high and low energy particlees: The mean energy of the particles corresponding to these curves is 1.74 times the injection energy when $R=2$, and 2.19 times the injection energy when $R=10$.

The particle densities of the se distributions are, for $R=2$,

$$
\dot{n}(t)=4.13 \times 10^{7} \frac{E^{3 / 4} J^{\frac{1}{2}}}{\left(1.000+0.1019 \log E_{e} \div 0.0340 \log n\right)^{\frac{1}{2}}} \int_{0}^{\infty} M(x, t) x^{2} d x,
$$

and for $\bar{R}=10$,

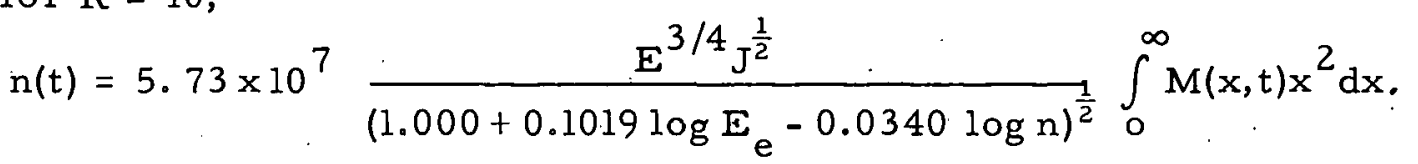

${ }^{17}$ This section is based upon numerical results obtained by G. Bing. 


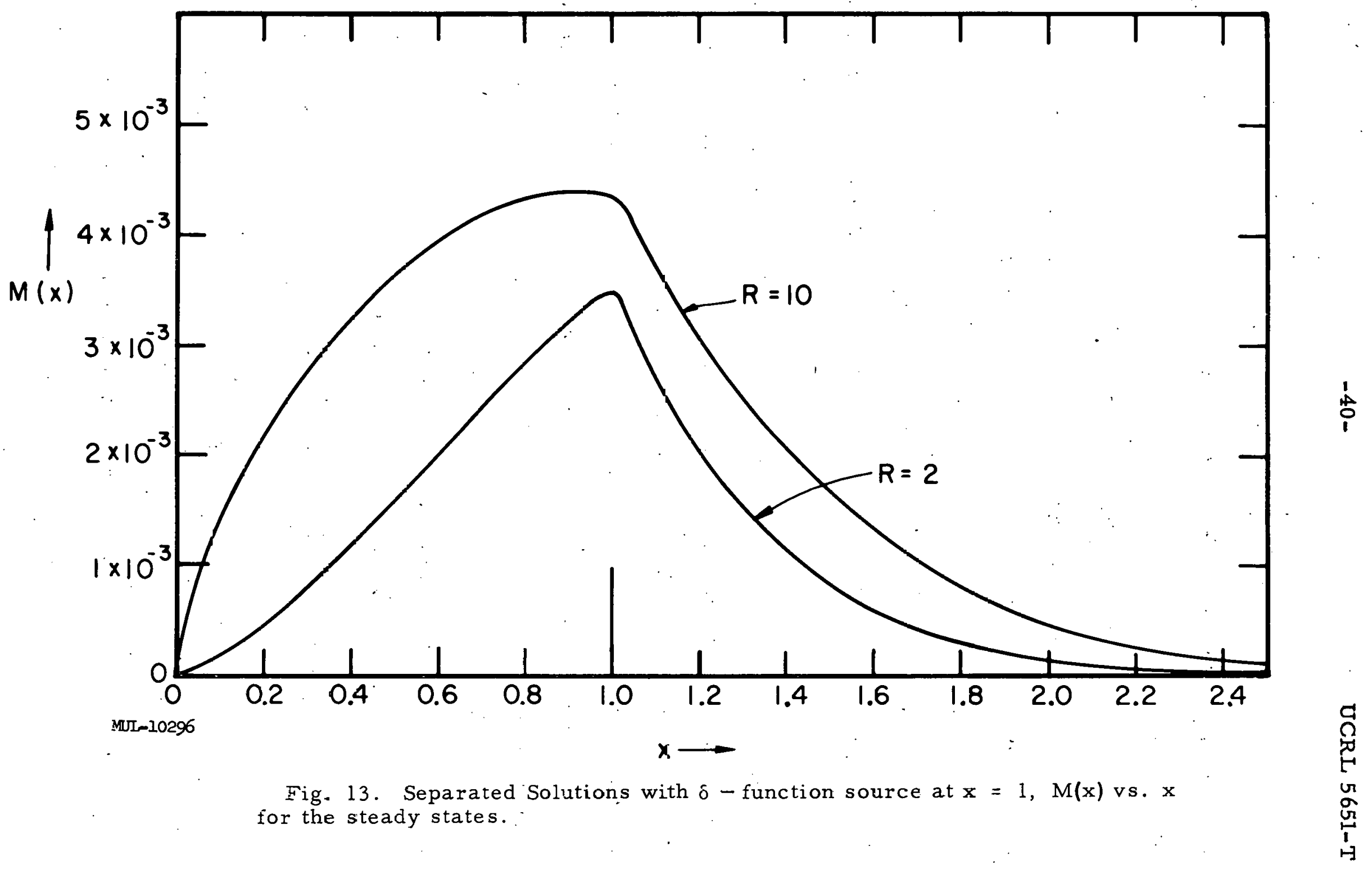


These expressions are corrected to give values of $n(t)$. that are consistent with the results of the unseparated initial-distribution type calculations. Equations (56) and (57) are derived in Appendix A-3.

At the steady state, for $R=2$,

$$
\int_{0}^{\infty} M(x) x^{2} d x=2.98 \times 10^{-3}
$$

and, for $\mathrm{R}=10$,

$$
\int_{0}^{\infty} M(x) x^{2} d x=5.63 \times 10^{-3}
$$

which.means that, for $R=2$,

$$
\mathrm{n}_{\substack{\text { steady } \\ \text { state }}}=1.231 \times 10^{5} \frac{\mathrm{E}^{3 / 4} \cdot \mathrm{J}^{\frac{1}{2}}}{\left(1.000+0.1019 \log \mathrm{E}_{\mathrm{e}}-0.0340 \log \mathrm{n}\right)^{\frac{1}{2}}}
$$

and for $R=10$,

$$
\mathrm{n}_{\substack{\text { steady } \\ \text { state }}}=3.23 \times 10^{5} \frac{\mathrm{E}^{3 / 4} \mathrm{~J}^{\frac{1}{2}}}{\left.1.000+0.1019 \log \mathrm{E}_{\mathrm{e}}-0.0340 \log \mathrm{n}\right)^{\frac{1}{2}}}
$$

Division of Eq. (61) by Eq. (60) gives a ratio of particle densities of 2.62 . The ratio of the logarithms of the mirror ratios is 3.3 to 1 , indicating that the steady-state particle density seems to vary less rapidly than $\log R$. A tabulation of some steady-state particle densities obtained with various injection current densities, assuming an injection energy $E$ of $100 \mathrm{kev}$ and an electron energy $\mathrm{E}_{\mathrm{e}}$ of $1 \mathrm{kev}$, is given in Table 5 .

\section{SUPPLEMENT ARY CALCULATIONS BASED ON THE UNSEPARATED SOLUTIONS}

A number of supplementary calculations were made from the unseparated solutions, among which were the relative number of particles remaining in the system, the mean energy of the particles, the relative numbers of particles 
TABLE 5. STEADY-STATE PARTIC.LE DENSITIES AT SOME SELECTED VALUES OF THE INJECTION CURRENT DENSITY: THE INJECTION ENERGY IS ASSUMED TO BE $100 \mathrm{KEV}$ AND THE ME AN ELECTRON ENERGY IS ASSUMED TO BE 1 KE.V

(These values are corrected as described in Appendix A-3 to be consistent with the results of the unseparated initial-distribution type calculations.)

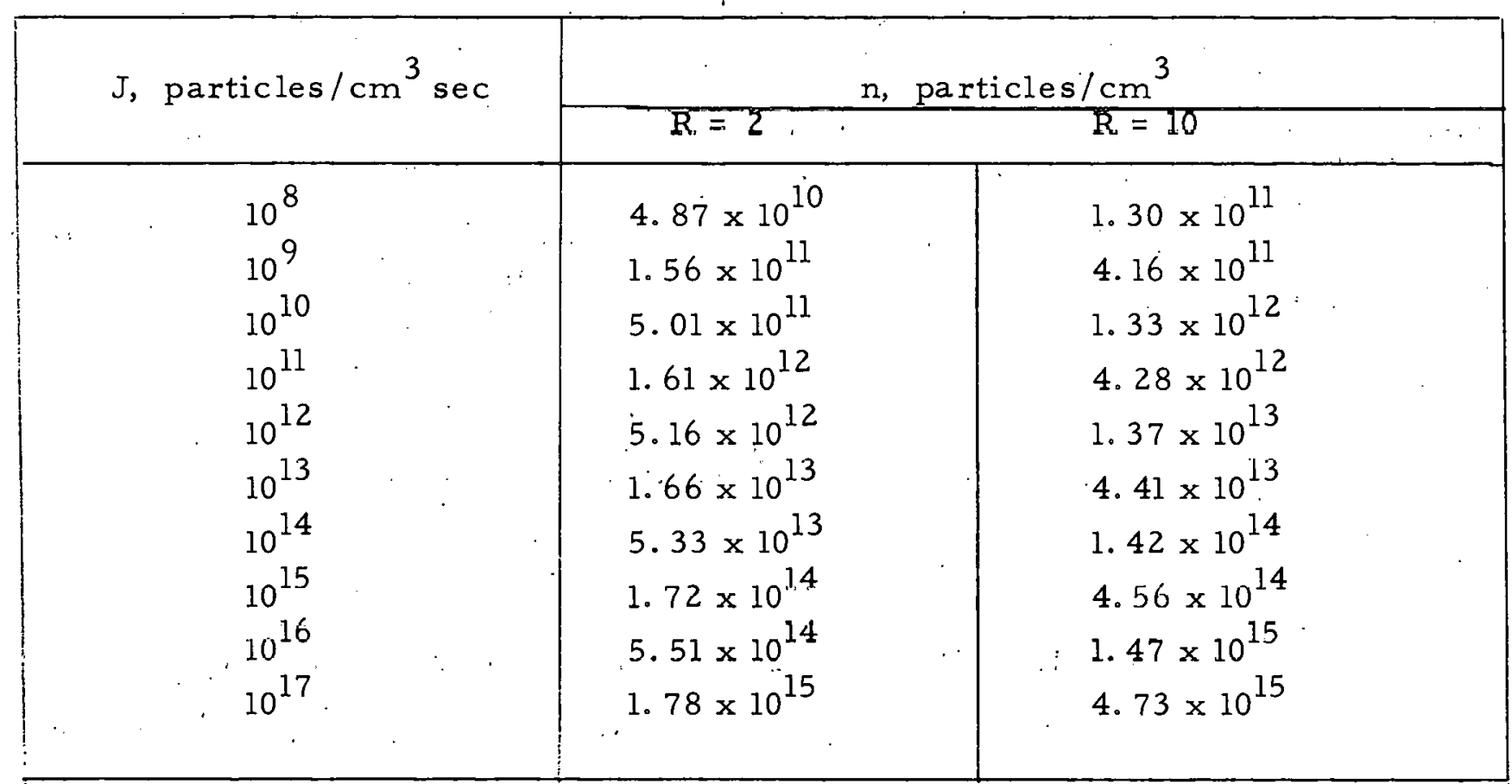

in certain energy ranges, the charge-exchange reaction rate, and fusion reaction rates. With the exception of the fusion reaction rates the supplementary calculations were made at each time step along with the computation of the distribution function. The calculation of the fusion reaction rates was so time consuming that it was made for only a few distributions, which were selected after the unseparated solutions had been allowed to undergo a considerable time-evolution:

The results of the calculations of the relative number of particles remaining in the system and of their mean energy have already been discussed 
in section IX and were given in Tables 2 and 3

\section{Relative Numbers of Particles in Certain Energy Ranges}

The results of the calculation of the relative number of particles in certain energy ranges are given in Table 6. Table 6 shows the absence of a time growth of much in the way of high-energy tails in both the normal mode and the peakedin-angle distribution.

TAB LE 6. RELATIVE NUMBERS OF PARTICLES IN CERTAIN ENERGY RANGES FOR THE NORMAL MODE AND PEAKED-IN-ANGLE UNSEPARAT ED SOLUTIONS

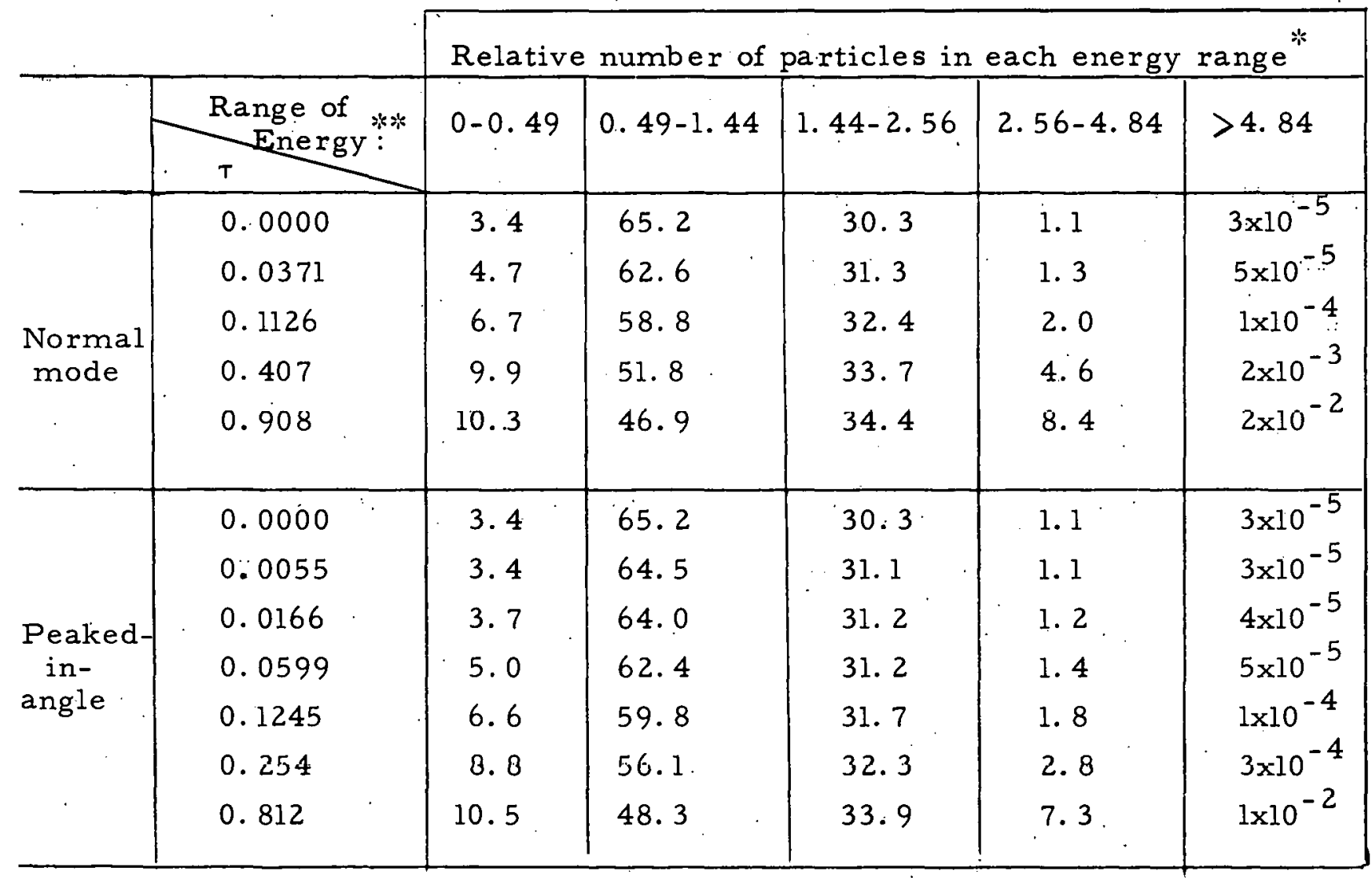

*Expressed in percent of total number of particles

***Expressed in percent of energy of peak of distribution 


\section{Rates of Charge-Exchange}

Because uncharged particles are not deflected by magnetic fields, any neutral particles present in mirror machines will travel freely from wall to wall and will consequently be cold. The presence of any significant quantity of cold neutral particles will provide an important avenue of escape for the hot ions. The mechanism of escape of a charged particle is to pick up a neutralizing electron from a cold neutral particle, after which it may travel unimpeded by the magnetic field to the: walls of the vessel.

The charge-exchange reaction rate is given by

$$
-\frac{d n}{d t}=n_{n} n \overline{\sigma v}
$$

where $n_{n}$ is the neutral particle density, $\sigma$ is the charge-exchange cross section, and

$$
\overline{\sigma v}=\frac{v_{0} \int_{0}^{\infty} \int_{0}^{\mu} R(\mu, x) \sigma(x) x^{3} d x d \mu}{\int_{0}^{\infty} \int_{0}^{\mu} R(\mu, x) x^{2} d x d \mu}
$$

In: Eq. (63) $\sigma$ is taken as $\sigma(\mathrm{x})$ rather than $\sigma\left(\mathrm{x}_{\mathrm{r}}\right)$, because the velocities of the cold neutral particles are presumed to be negligibly small in comparison with the ionic velocities.

One may see by kq. (62) that for sufficiently high neutral particle densities, the charge-exchange mechanism can be the dominant means for losing the ions from the mirror machine systems.

Charge-exchange reaction rates, as represented by $\overline{\sigma v}$, were computed at the following energies (corresponding to $x=1$ in the distribution function): $1,2,4,8,16,32,65,125,250,500$, and $1000 \mathrm{kev}$.

The cross sections used in computing the $\overline{\sigma \mathrm{v}}$ 's were obtained from measurements made on protons impinging on $\mathrm{H}_{2}$ gas. According to Jackson 
and Schiff ${ }^{18}$ the only effect of changing the masses, for a given velocity, is to change the angular distribution of the resulting scatters.

For protons from 125 volts to $4 \mathrm{kev}$ the data of Stedeford and Hasted 19 were used. Below 125 volts the cross section.was estimated to be about $1.5 \times 10^{-17} \mathrm{~cm}^{2}$ on the basis of a curve credited to Wolf in the paper of Stedeford and Hasted. ${ }^{19}$ This figure may be somewhat in error, but the values of $F$ are sufficiently small at speeds calling for this value of the cross section that it should not lead to a serious error in $\overline{\sigma v}$, particularly at $8 \mathrm{kev}$ and above. From $4 \mathrm{kev}$ to $50 \mathrm{kev}$ the data of Stier and Barnett 20 were used. The data of Barnett and Reynolds ${ }^{21}$ were used in the 50-to 1000 -kev region. Above $1000 \mathrm{kev}$ the $1 / \mathrm{E}^{6}$ variation of the cross section indicated by the asymptotic form of the Brinkman and Kramers ${ }^{22}$ calculation was used.

The $\overline{\sigma v^{\prime}}$ s for several distributions are given in Table 7 for the energies mentioned above. It may be seen that except for energies greater than about $250 \mathrm{kev}$, the $\frac{\cdots v}{\sigma \cdot \mathrm{l}} \mathrm{s}$ are relatively insensitive to the exact shape of the distribution function. The cross sections are dropping very rapidly in the greaterthan-250-kev region, as may be seen by inspecting the $\delta$-function-in-speedcolumn of Table 7. Consequently, distributions that are spread out in speed will produce markedly greater $\overline{\dot{\sigma} v}$ 's in the high-energy region than will peakedin-speed distributions.

18 Jackson and Schiff, Phys. Rev. 89, 359 (1953).

19 J. B. H. Stedeford and J. B. Hasted, Proc. Roy. Soc. (London) A227, 466 (1955).

${ }^{20}$ P. M. Stier and C. F. Barnett, Phys. Rev. 103, 896 (1956).

${ }^{21}$ C. F. Barnett and H. K. Reynolds, Phys. Rev. 10\%, 355 (1958).

${ }^{22}$ H. C. Brinkman and H. A. Kramers, Proc. Acad. Sci. Amsterdam 33 , 973 (1930). 
TABLE 7. TABULATION OF $\overline{\sigma \mathrm{V}^{\prime}} \mathrm{S}$ FOR THE CHARGE-EXCHANGE REACTION BETWEEN DEUTERIUM IONS AND DEUTERIUM ATOMS IN DEUTERIUM GAS (in $\mathrm{cm}^{3} / \mathrm{sec}$ ).

\begin{tabular}{|c|c|c|c|c|c|c|}
\hline \multirow[t]{2}{*}{$\mathrm{E}, \mathrm{kev}$} & \multirow{2}{*}{$\begin{array}{c}\delta \text {-function } \\
\text { in } \\
\text { speed }\end{array}$} & \multicolumn{3}{|c|}{ Normal Mode } & \multicolumn{2}{|c|}{ Peaked-in-Angle } \\
\hline & & $T=0.908$ & $T=0.1126$ & $\tau=0$ & $\tau=0$ & $T=0.254$ \\
\hline 1 & $4.6 \times 10^{-9}$ & 7. $0 \times 10^{-9}$ & 6. $2 \times 10^{-9}$ & $6.1 \times 10^{-9}$ & $6.1 \times 10^{-9}$ & 6. $2 \times 10^{-9}$ \\
\hline$\dot{2}$ & $1.1 \times 10^{-8}$ & 1. $4 \times 10^{-8}$ & 1. $3 \times 10^{-8}$ & 1. $3 \times 10^{-8}$ & 1. $3 \times 10^{-8}$ & 1. $3 \times 10^{-8}$ \\
\hline 4 & $2.1 \times 10^{-8}$ & $2.5 \times 10^{8}$ & 2. $4 \times 10^{-8}$ & 2. $4 \times 10^{-m 8}$ & 2. $4 \times 10^{-8}$ & 2. $4 \times 10^{-8}$ \\
\hline 8 & 3. $4 \times 10^{-8}$ & 3. $9 \times 10^{-8}$ & $3.7 \times 10^{-8}$ & 3. $8 \times 10^{-8}$ & 3. $8 \times 10^{-8}$ & 3. $7 \times 10^{-8}$ \\
\hline 16 & $5.0 \times 10^{-8}$ & $5.1 \times 10^{-8}$ & $5.1 \times 10^{-8}$ & 5. $2 \times 10^{-8}$ & 5. $2 \times 10^{-8}$ & $5.1 \times 10^{-8}$ \\
\hline 32 & $6.1 \times 10^{-8}$ & $5.1 \times 10^{-8}$ & $5.5 \times 10^{-8}$ & $5.6 \times 10^{-8}$ & $5.6 \times 10^{-8}$ & 5. $4 \times 10^{-8}$ \\
\hline 65 & $4.5 \times 10^{-8}$ & 3. $4 \times 10^{-8}$ & $3.7 \times 10^{-8}$ & 3. $8 \times 10^{-8}$ & 3. $8 \times 10^{-8}$ & 3. $7 \times 10^{-8}$ \\
\hline 125 & 1. $7 \times 10^{-8}$ & 1. $7 \times 10^{-8}$ & 1. $7 \times 10^{-8}$. & $1.6 \times 10^{-8}$ & $1.6 \times 10^{-8}$ & 1. $8 \times 10^{-8}$ \\
\hline 250 & 2. $8 \times 10^{-9}$ & $6.1 \times 10^{-9}$ & $5.0 \times 10^{-9}$ & 3. $8 \times 10^{-9}$ & $3.8 \times 10^{-9}$ & $5.8 \times 10^{-9}$ \\
\hline 500 & 2. $1 \times 10^{-10}$ & 1. $7 \times 10^{-9}$ & 1. $0 \times 10^{-9}$ & $5.1 \times 10^{-10}$ & 5. $1 \times 10^{-10}$ & 1. $4 \times 10^{-9}$ \\
\hline 1000 & $5.9 \times 10^{-12}$ & 4. $6 \times 10^{-10}$ & 1. $9 \times 10^{-10}$ & $4.8 \times 10^{-11}$ & 4. $8 \times 10^{-11}$ & $3.1 \times 10^{-10}$ \\
\hline
\end{tabular}

A sample calculation will illustrate the sort: of vacuum requirements necessary to keep the charge-exchange loss rate less than the end-loss rate. If one assumes, for example, that one has a distribution corresponding to the normal mode at $\tau=0.908$, that the energy corresponding to $x=1$ is $125 \mathrm{kev}$, that the electron energy $\mathrm{E}_{\mathrm{e}}$ is $1 \mathrm{kev}$, and that the deuteron density $1 \mathrm{~s} 10^{1.4} \mathrm{~cm}^{-3}$, then by Table 2 and Eqs. (54) and (35) the end-loss rate will be

$$
-\frac{\mathrm{dn}}{\mathrm{dt}}=1.5 \times 10^{14} \text { deuterons } / \mathrm{cm}^{3} \mathrm{sec} \text {. }
$$

If the charge-exchange loss rate is to be less than the end-loss rate, then from Eq. (62),

$$
\mathrm{n}_{\mathrm{n}} \mathrm{n} \frac{\partial}{\sigma \mathrm{v}}<1.5 \times 10^{14}
$$


Introduction of the appropriate value of $\overline{\sigma v}$ from Table $7,1.7 . x: 10^{-8}$, then gives for the neutral deuterium atom density.

$$
\mathrm{n}_{\mathrm{n}}<8.8 \times 10^{7} \text { neutral deuterium atoms } / \mathrm{cm}^{3}
$$

If the bulk of the deuterium is in the form of $\mathrm{D}_{2}$, the molecular density would have to be less than $4.4 \times 10^{7}$ molecules $/ \mathrm{cm}^{3}$. If one assumes the neutral $D_{2}$ gas to be at a temperature of $1000^{\circ} \mathrm{K}$, this particle density would correspond to a pressure of $4.6 \times 10^{-9} \mathrm{~mm} \mathrm{Hg}$.

\section{Fusion Reaction Rates}

The fusion reaction rate is given by

$$
-\frac{\mathrm{dn}}{\mathrm{dt}}=\frac{1}{2} \mathrm{n}^{2} \frac{\dot{\sigma}}{\sigma \mathrm{v}}
$$

for the DD reaction and by

$$
-\frac{\mathrm{dn}}{\mathrm{dt}}=\mathrm{n}_{\mathrm{D}^{\mathrm{n}} \mathrm{T}} \overline{\sigma \mathrm{v}}
$$

for the DT reaction. In the case where $n_{D}=n_{T}$, Eq. (68) may be written

$$
-\frac{\mathrm{dn}}{\mathrm{dt}}=\frac{1}{4} \mathrm{n}^{2} \overline{\sigma \mathrm{v}}
$$

where $\mathrm{n}=\mathrm{n}_{\mathrm{D}}+\mathrm{n}_{\mathrm{T}}$.

The $\overline{\sigma v}$ s for the $\mathrm{DD}$ and $\mathrm{DT}$ reactions were calculated for energies (referring to the energy of the $x=1$, particles) of $1,2,4,8,16,32,65,125$, 250, 500, and $1000 \mathrm{kev}$ for Maxwellian and several peaked-in-angle and normal mode distributions.

The $\overline{\sigma v}$ 's were obtained by substituting appropriately tabulated values of the ecross section $\sigma$ into

$$
\overline{\pi v}=\frac{2 \pi v \int_{0}^{\infty} 2 \int_{0}^{\mu} x^{2} F(\mu, x) \int_{0}^{\infty} \int_{-\mu_{R}}^{\mu} R \int_{0}^{\pi} x^{\prime}{ }^{2} F\left(\mu^{\prime}, x^{\prime}\right) x_{r} \sigma\left(\dot{x}{ }_{r}\right) d \phi d \mu^{\prime} d x^{\prime} d \mu d x}{\left[2 \pi \int_{0}^{\infty} 2 \int_{0}^{\mu} x^{2} F(\mu, x) d \mu d \dot{x}\right]^{2}}
$$


(See Part B for a discussion of the computational details: In turning to Part

B note that $a\left(x_{r}^{2}\right) \cdot\left(v_{o} / \sqrt{E}\right) \equiv v_{r} \sigma\left(v_{r}\right)=v_{o} x_{r} \sigma\left(v_{r}\right)$. The transition from Eq. (70) to the similar equation of Sec. VIII of Part B should then be apparent.) The cross sections used in this computation were those of J. L. Tuck ${ }^{23}$. In order to obtain the $\overline{\sigma v}$ 's at 500 and $1000 \mathrm{kev,} \mathrm{Tuck's} \mathrm{curves} \mathrm{had} \mathrm{to} \mathrm{be} \mathrm{extrapolated}$ slightly. However, the extrapolated values of $\sigma$ used in the computation were $\therefore$.

less than the largest values and were multiplied by such small values of $F$ that any errors in the extrapolation: should have had negligible effects on the calculated $\overline{\sigma^{\prime}} \mathrm{s}$.

The results of the $\overline{\sigma v}$ computations are given in Tables 8 through 13. It should be remarked that the computing machine code could handle only one velocity distribution, and consequently the $\overline{\sigma \mathrm{v}}$ 's for the DT reaction were computed with the assumption that all particles were of mass 3 . This assumption leads to mean relative velocities of the order of $10 \%$ too low for the DT mixture. The DT results in Tables 8 and 9 have been corrected for this effect, i.e., values corresponding to the estimated true mean relative velocities have been entered in the tables. In the low-energy region the $\overline{\sigma v}$ 's are seen to be quite sensitive to the shape of the particle distribution function. Consequently this method of correcting the DT $\overline{\sigma V}$ is might not be sufficiently accurate for low energies except that the low-energy $\sigma^{\prime}$ s themselves are not accurately known. In the higher energy regions the $\overline{\sigma v}$ 's . are less sensitive to the precise form of the distribution functions and this correction should be adequate.

23 J. L. Tuck, Los Alamos, LA-1190, May 10, 1951. 
Tables 8 through 13 show that the fusion reaction rates are relatively insensitive to the shape of the distribution functions except at low energies. At low energies the fusion cross sections are changing so rapidly that the presence or absence of a Maxwellian tail in the distribution can have approximately a fifty-fold effect on the reaction rates.

Arnold, Phillips, Sawyer, Stovall, and Tuck ${ }^{24}$ present somewhat higher values of the DT cross sections below $120 \mathrm{kev}$ than did Tuck. 23 W. B.

Thompson ${ }^{25}$ obtained a value of $2.6 \times 10^{-16} \mathrm{~cm}^{3} / \mathrm{sec}$ for the $\mathrm{DT} \overline{\sigma \mathrm{v}}$ at $100 \mathrm{kev}$ based on the cross sections of Arnold et al. His value seems to be somewhat low in comparison with Tuck's $^{23}$ and the present calculation. However, all three calculations give about the same "value for DT at $10 \mathrm{kev}$. Thompson gives a $\overline{\sigma v}$ of $9.3 \times 10^{-21} \mathrm{~cm}^{3} / \mathrm{sec}$ for DT at $1 \mathrm{kev}$, to be compared with Tuck's $4.0 \times 10^{-21}$ and the present calculations $1.2 \times 10^{-21}$. The cross sections change so rapidly in this energy region, however, that one should probably not anticipate better than order-or-magnitude agreement.

\section{ACKNOW LEDGMENT}

The author would like to express his sincere appreciation to George Bing, W. A. Newcomb, and T. G. Northrop for many helpful discussions. He would also like to express his appreciation to Marlene Carr and to Susan Conniff for their assistance in carrying out the computations.

24 Arnold, Phillips, Sawyer, Stovall, and Tuck, Phys. Rev. 93, 483 (1954). ${ }^{25}$ W. B. Thompson, Proc. Phys. Soc. B70, 1 (1957). 
TABLE 8. DD $\overline{\sigma v}$ 's IN $\mathrm{cm}^{3} / \mathrm{sec}$ FOR MAXWELLIAN, NORMAL MODE AT $\tau=0$, AND PEAKED-IN-ANGLE AT $\tau=0$ DISTRIBUTIONS:

Note: The energies listed below correspond to those particles of the distributions for which $x=1$. In the Maxwellian case the speed distribution is $\mathrm{e}^{-\mathrm{x}^{2}}$; in the normal mode and peaked-in-angle distributions the speed distribution at $\tau=0$ is $e^{-10(x-1)^{2}}$. The mean energy of the Maxwellian distribution is 1.5 times the energy entry in the table. The mean energies of the normal modo and poalced-in-anglo dietributione at $T=0$ are $1.25^{\circ}$ times the entry in the table.

\begin{tabular}{|c|c|c|c|c|}
\hline E, kev & $\begin{array}{c}\text { Tuck's } \\
\text { Maxwellian results }\end{array}$ & $\begin{array}{c}\text { Present Maxwellian } \\
\text { results }\end{array}$ & $\begin{array}{c}\text { Normal Mode } \\
\tau=0\end{array}$ & $\begin{array}{c}\text { Peaked-in } \\
\text { angle } \tau=0\end{array}$ \\
\hline 1 & $2.0 \times 10^{-22}$ & $1.5 \times 10^{-22}$ & $4.1 \times 10^{-24}$ & $3.7 \times 10^{-24}$ \\
2 & $6: 0 \times 10^{-21}$ & $5.3 \times 10^{-21}$ & $5.0 \times 10^{-22} \cdot$ & $4.0 \times 10^{-22}$ \\
4 & $1.0 \times 10^{-19}$ & $8.8 \times 10^{-20}$ & $1.8 \times 10^{-20}$ & $1.4 \times 10^{-20}$ \\
8 & $8.6 \times 10^{-19}$ & $7.4 \times 10^{-19}$ & $3.0 \times 10^{-19}$ & $2.1 \times 10^{-19}$ \\
16 & $3.8 \times 10^{-18}$ & $3.6 \times 10^{-18}$ & $2.1 \times 10^{-18}$ & $1.4 \times 10^{-18}$ \\
32 & $1.2 \times 10^{-17}$ & $1.2 \times 10^{-17}$ & $8.4 \times 10^{-18}$ & $5.2 \times 10^{-18}$ \\
65 & $3.1 \times 10^{-17}$ & $2.9 \times 10^{-17}$ & $2.3 \times 10^{-17}$ & $1.4 \times 10^{-17}$ \\
125 & $6.4 \times 10^{-17}$ & $5.6 \times 10^{-17}$ & $4.6 \times 10^{-17}$ & $2.7 \times 10^{-17}$ \\
250 & $1.1 \times 10^{-16}$ & $1.0 \times 10^{-16}$ & $8.8 \times 10^{-17}$ & $5.1 \times 10^{-17}$ \\
500 & $1.7 \times 10^{-16}$ & $1.6 \times 10^{-16}$ & $1.5 \times 10^{-16}$ & $8.4 \times 10^{-17}$ \\
1000 & $2.5 \times 10^{-16}$ & $2.4 \times 10^{-16}$ & $2.2 \times 10^{-16}$ & $1.2 \times 10^{-16}$ \\
\hline
\end{tabular}


TABLE 9. DT $\overline{\sigma v}$ 's IN $\mathrm{cm}^{3} / \mathrm{sec}$ FOR MAXWELLIAN; NORMAL MODE $\dot{A T} \tau=0$, AND PEAKED-IN-ANGLE AT $\tau=0$ DISTRIBUTIONS

(See note with Table 8.)

\begin{tabular}{|c|c|c|c|c|c|}
\hline \multicolumn{6}{|c|}{ DT } \\
\hline $\mathrm{E}_{\mathrm{g}} \mathrm{kev}$ & $\begin{array}{l}\text { Tuck's } 23 \\
\text { Maxwellian results }\end{array}$ & $\begin{array}{l}\text { Present } \\
\text { Maxwellian }\end{array}$ & results & $\begin{array}{l}\text { Normal mode } \\
\tau=0\end{array}$ & $\begin{array}{l}\text { Peaked-in- } \\
\text { angle } \tau=0\end{array}$ \\
\hline 1 & 4. $0 \times 10^{-21}$ & 1. $2 \times 10^{-21}$ & & 1. $0 \times 10^{-22}$ & $8.0 \times 10^{-23}$ \\
\hline 2 & $1.6 \times 10^{-19}$ & 1. $8 \times 10^{-19}$ & & 1. $5 \times 10^{-20}$ & 1. $2 \times 10^{-20}$ \\
\hline 4 & 3. $4 \times 10^{-18}$ & 3. $3 \times 10^{-18}$ & & $6.0 \times 10^{-19}$ & 4. $7 \times 10^{-19}$ \\
\hline 8 & 4. $0 \times 10^{-17}$ & 4. $0 \times 10^{-17}$ & & 1. $3 \times 10^{-17}$ & $9.6 \times 10^{-18}$ \\
\hline 16 & 2. $4 \times 10^{-16}$ & 2. $2 \times 10^{-16}$ & & 1. $5 \times 10^{-16}$ & 1. $0 \times 10^{-16}$ \\
\hline 32 & $6.0 \times 10^{-16}$ & $5.8 \times 10^{-16}$ & & $6.4 \times 10^{-16^{\prime}}$ & 4. $0 \times 10^{-16}$ \\
\hline 65 & 8. $4 \times 10^{-16}$ & 8. $0 \times 10^{-16}$ & & $9.8 \times 10^{-16}$ & $5.1 \times 10^{-16}$ \\
\hline 125 & $\ldots$ & $6.9 \times 10^{-16}$ & & 8. $2 \times 10^{-16}$ & 4. $0 \times 10^{-16}$ \\
\hline 250 & $-\ldots$ & 4. $9 \times 10^{-16}$ & & 5. $8 \times 10^{-16}$ & 3. $0 \times 10^{-16}$ \\
\hline 500 & $-\cdots$ & $3.1 \times 10^{-16}$ & & 3. $9 \times 10^{-16}$ & 2. $4 \times 10^{-16}$ \\
\hline 1000 & --- & 2. $0 \times 10^{-16}$ & & 2. $7 \times 10^{-16}$ & 1. $9 \times 10^{-16}$ \\
\hline
\end{tabular}


TABLE 10: VARIATION WITH T OF NORMAL MODE $\overline{\sigma \mathrm{v}}$ 's FOR THE DD REACTION IN COMPARISON WITH THE MAXWELLIAN $\overline{\sigma V}$. (See note with Table 8)

$\mathrm{DD} \overline{\sigma \mathrm{v}}$ 's $\mathrm{cm}^{3} / \mathrm{sec}$

\begin{tabular}{|c|c|c|c|c|c|}
\hline E, kev & $\tau=0.0000$ & $\tau=0.1126$ & $\tau=0.407$ & $\tau=0.908$ & $\begin{array}{l}\text { Present } \\
\text { Maxwellian } \\
\text { calculation }\end{array}$ \\
\hline 1 & $1.1 \times 10^{-24}$ & $6.5 \times 10^{-24}$ & $1.4 \times 10^{-23}$ & $2.8 \times 10^{-23}$ & $1.5 \times 10^{-2 L}$ \\
2 & $5.0 \times 10^{-22}$ & $6.3 \times 10^{-22}$ & $9.8 \times 10^{-22}$ & $1.6 \times 10^{-21}$ & $5.3 \times 10^{-21}$ \\
4 & $1.8 \times 10^{-20}$ & $2.2 \times 10^{-20}$ & $2.9 \times 10^{-20}$ & $4.3 \times 10^{-20}$ & $8.8 \times 10^{-20}$ \\
8 & $3.0 \times 10^{-19}$ & $3.2 \times 10^{-19}$ & $3.9 \times 10^{-19}$ & $5.0 \times 10^{-19}$ & $7.4 \times 10^{-19}$ \\
16 & $2.1 \times 10^{-18}$ & $2.3 \times 10^{-18}$ & $2.4 \times 10^{-18}$ & $3.0 \times 10^{-18}$ & $3.6 \times 10^{-18}$ \\
32 & $8.4 \times 10^{-18}$ & $8.4 \times 10^{-18}$ & $9.0 \times 10^{-18}$ & $1.0 \times 10^{-17}$ & $1.2 \times 10^{-17}$ \\
65 & $2.3 \times 10^{-17}$ & $2.3 \times 10^{-17}$ & $2.4 \times 10^{-17}$ & $2.6 \times 10^{-17}$ & $2.9 \times 10^{-17}$ \\
125 & $4.6 \times 10^{-17}$ & $4.6 \times 10^{-17}$ & $4.8 \times 10^{-17}$ & $5.2 \times 10^{-17}$ & $5.6 \times 10^{-17}$ \\
250 & $8.8 \times 10^{-17}$ & $8.8 \times 10^{-17}$ & $9.0 \times 10^{-17}$ & $9.5 \times 10^{-17}$ & $1.0 \times 10^{-16}$ \\
500 & $1.5 \times 10^{-16}$ & $1.5 \times 10^{-16}$ & $1.5 \times 10^{-16}$ & $1.5 \times 10^{-16}$ & $1.6 \times 10^{-16}$ \\
1000 & $2.2 \times 10^{-16}$ & $2.2 \times 10^{-16}$ & $2.2 \times 10^{-16}$ & $2.2 \times 10^{-16}$ & $2.4 \times 10^{-16}$ \\
\hline
\end{tabular}


TABLE 11. VARIATION WITH T OF NORMAL MODE $\overline{\sigma v}{ }^{\prime}$ 's FOR THE DT REACTION IN COMPARISON WITH THE MAXWELLIAN $\overline{\sigma v}$.

(See note with Table 8.)

$\mathrm{DT} \overline{\sigma v^{\prime} \mathrm{s}} \mathrm{cm}^{3} / \mathrm{sec}$

\begin{tabular}{|c|c|c|c|c|c|}
\hline E, kev & $\tau=0.0000$ & $\tau=0.1126$ & $\tau=0.407$ & $\tau=0.908$ & $\begin{array}{l}\text { Present Max- } \\
\text { wellian calc. }\end{array}$ \\
\hline 1 & $1.0 \times 10^{-22}$ & $1.5 \times 10^{-22}$ & $3.5 \times 10^{-22}$ & $7.0 \times 10^{-22}$ & $1.2 \times 10^{-21}$ \\
2 & $1.5 \times 10^{-20}$ & $1.8 \times 10^{-20}$ & $2.9 \times 10^{-20}$ & $5.0 \times 10^{-20}$ & $1.8 \times 10^{-19}$ \\
4 & $6.0 \times 10^{-19}$ & $7.2 \times 10^{-19}$ & $1.0 \times 10^{-18}$ & $1.6 \times 10^{-18}$ & $3.3 \times 10^{-18}$ \\
8 & $1.3 \times 10^{-17}$ & $1.5 \times 10^{-17}$ & $2.0 \times 10^{-17}$ & $2.5 \times 10^{-17}$ & $4.0 \times 10^{-17}$ \\
16 & $1.5 \times 10^{-16}$ & $1.6 \times 10^{-16}$ & $1.8 \times 10^{-16}$ & $2.2 \times 10^{-16}$ & $2.2 \times 10^{-16}$ \\
32 & $6.4 \times 10^{-16}$ & $6.7 \times 10^{-16}$ & $6.7 \times 10^{-16}$ & $6.9 \times 10^{-16}$ & $5.8 \times 10^{-16}$ \\
65 & $9.8 \times 10^{-16}$ & $9.7 \times 10^{-16}$ & $9.4 \times 10^{-16}$ & $9.2 \times 10^{-16}$ & $8.0 \times 10^{-16}$ \\
125 & $8.2 \times 10^{-16}$ & $8.2 \times 10^{-16}$ & $8.2 \times 10^{-16}$ & $8.1 \times 10^{-16}$ & $6.9 \times 10^{-16}$ \\
250 & $5.8 \times 10^{-16}$ & $5.9 \times 10^{-16}$ & $5.9 \times 10^{-16}$ & $5.7 \times 10^{-16}$ & $4.9 \times 10^{-16}$ \\
500 & $3.9 \times 10^{-16}$ & $3.9 \times 10^{-16}$ & $3.8 \times 10^{-16}$ & $3.8 \times 10^{-16}$ & $3.1 \times 10^{-16}$ \\
1000 & $2.7 \times 10^{-16}$ & $2.5 \times 10^{-16}$ & $2.5 \times 10^{-16}$ & $2.4 \times 10^{-16}$ & $2.0 \times 10^{-16}$ \\
\hline
\end{tabular}


TAB LE 12

VARIATION WITH $T$ OF THE PEAKED-IN-ANGLE $\overline{\sigma V}$ 's FOR THE DD REACTION IN COMPARISON WITH THE MAXWELLIAN $\overline{\sigma v}$.

(See note with Table 8.)

\begin{tabular}{|c|c|c|c|c|c|}
\hline \multicolumn{7}{|c|}{$\mathrm{DD} \bar{\sigma} \mathrm{v}^{\prime} \mathrm{s} \mathrm{cm}^{3} / \mathrm{sec}$} & $\begin{array}{c}\text { Present } \\
\text { Maxwellian } \\
\text { calculation }\end{array}$ \\
\hline E, kev & $\tau=0.0000$ & $\tau=0.0166$ & $\tau=0.0599$ & $\gamma=0.1245$ & $\cdots$ \\
\hline 1 & $3.7 \times 10^{-24}$ & $4.3 \times 10^{-24}$ & $5.1 \times 10^{-24}$ & $7.5 \times 10^{-24}$ & $1.5 \times 10^{-27}$ \\
2 & $4.0 \times 10^{-22}$ & $4.4 \times 10^{-22}$ & $5.1 \times 10^{-22}$ & $6.6 \times 10^{-22}$ & $5.3 \times 10^{-21}$ \\
4 & $1.4 \times 10^{-20}$ & $1.6 \times 10^{-20}$ & $1.8 \times 10^{-20}$ & $2.2 \times 10^{-20}$ & $8.8 \times 10^{-20}$ \\
8 & $2.1 \times 10^{-19}$ & $2.3 \times 10^{-19}$ & $2.7 \times 10^{-19}$ & $3.0 \times 10^{-19}$ & $7.4 \times 10^{-19}$ \\
16 & $1.4 \times 10^{-18}$ & $1.6 \times 10^{-18}$ & $1.8 \times 10^{-18}$ & $2.0 \times 10^{-18}$ & $3.6 \times 10^{-18}$ \\
32 & $5.2 \times 10^{-18}$ & $5.9 \times 10^{-18}$ & $6.7 \times 10^{-18}$ & $7.6 \times 10^{-18}$ & $1.2 \times 10^{-17}$ \\
65 & $1.4 \times 10^{-17}$ & $1.5 \times 10^{-17}$ & $1.8 \times 10^{-17}$ & $2.0 \times 10^{-17}$ & $2.9 \times 10^{-17}$ \\
125 & $2.7 \times 10^{-17}$ & $3.1 \times 10^{-17}$ & $3.6 \times 10^{-17}$ & $4.1 \times 10^{-17}$ & $5.6 \times 10^{-17}$ \\
250 & $5.1 \times 10^{-17}$ & $5.8 \times 10^{-17}$ & $6.7 \times 10^{-17}$ & $7.7 \times 10^{-17}$ & $1.0 \times 10^{-16}$ \\
500 & $8.4 \times 10^{-17}$ & $9.6 \times 10^{-17}$ & $1.1 \times 10^{-16}$ & $1.3 \times 10^{-16}$ & $1.6 \times 10^{-16}$ \\
1000 & $1.2 \times 10^{-16}$ & $1.4 \times 10^{-16}$ & $1.6 \times 10^{-16}$ & $1.9 \times 10^{-16}$ & $2.4 \times 10^{-16}$ \\
\hline
\end{tabular}


TABLE 13. VARIATION WITH $T$ OF THE PEAKED-IN-ANGLE $\overline{\sigma V}$ 's FOR THE DT REACTION IN COMPARISON WITH THE MAXWELLIAN $\overline{\sigma V}$.

(See note with Table 8.)

$$
\text { DT } \overline{\sigma v^{\prime}} \mathrm{s} \mathrm{cm}^{3} / \mathrm{sec}
$$

\begin{tabular}{|c|c|c|c|c|c|}
\hline E, kev & $\tau=0.0000$ & $\tau=0.0166$ & $\tau=0.0599$ & $\tau=0.1245$ & $\begin{array}{l}\text { Present } \\
\text { Maxwellian Calc }\end{array}$ \\
\hline 1 & $8.0 \times 10^{-23}$ & $9.5 \times 10^{-23}$ & $1.3 \times 10^{-22}$ & $1.7 \times 10^{-22}$ & $1.2 \times 10^{-21}$ \\
2 & $1.2 \times 10^{-20}$ & $1.3 \times 10^{-20}$ & $1.5 \times 10^{-20}$ & $2.0 \times 10^{-20}$ & $1.8 \times 10^{-19}$ \\
4 & $4.7 \times 10^{-19}$ & $4.9 \times 10^{-19}$ & $6.0 \times 10^{-19}$ & $7.2 \times 10^{-19}$ & $3.3 \times 10^{-18}$ \\
8 & $9.6 \times 10^{-18}$ & $1.1 \times 10^{-17}$ & $1.3 \times 10^{-17}$ & $1.5 \times 10^{-17}$ & $4.0 \times 10^{-17}$ \\
16 & $1.0 \times 10^{-16}$ & $1.2 \times 10^{-16}$ & $1.4 \times 10^{-16}$ & $1.5 \times 10^{-16}$ & $2.2 \times 10^{-16}$ \\
32 & $4.0 \times 10^{-16}$ & $4.6 \times 10^{-16}$ & $5.1 \times 10^{-16}$ & $5.8 \times 10^{-16}$ & $5.8 \times 10^{-16}$ \\
65 & $5.1 \times 10^{-16}$ & $5.8 \times 10^{-16}$ & $6.9 \times 10^{-16}$ & $8.0 \times 10^{-16}$ & $8.0 \times 10^{-16}$ \\
125 & $4.0 \times 10^{-16}$ & $4.8 \times 10^{-16}$ & $5.8 \times 10^{-16}$ & $7.0 \times 10^{-16}$ & $6.9 \times 10^{-16}$ \\
250 & $3.0 \times 10^{-16}$ & $3.6 \times 10^{-16}$ & $4.4 \times 10^{-16}$ & $5.3 \times 10^{-16}$ & $4.9 \times 10^{-16}$ \\
500 & $2.4 \times 10^{-16}$ & $2.8 \times 10^{-16}$ & $3.4 \times 10^{-16}$ & $3.7 \times 10^{-16}$ & $3.1 \times 10^{-16}$ \\
1000 & $1.9 \times 10^{-16}$ & $2.2 \times 10^{-16}$ & $2.4 \times 10^{-16}$ & $2.6 \times 10^{-16}$ & $2.0 \times 10^{-16}$ \\
\hline
\end{tabular}




\section{APPENDIX A-1}

A demonstration that $\underline{v} \times \underline{B} \cdot \underline{\nabla}_{v} f=0$ implies an azimuthally uniform velocity distribution function, i. e., $\frac{\partial f}{\partial \phi}=0$.

Let $e_{1}$ be a unit vector in the direction of the magnetic field. Then, $\underline{B}=\underline{B e}_{1}$. Let $\underline{\mathbf{e}}_{2}$ and $\underline{\mathbf{e}}_{3}$ be two more unit vectors forming with $\underline{e}_{1}$ a mutually orthogonal set. $\because$ Then,

$B\left(\underline{v}_{\underline{e}_{1}}\right) \cdot \underline{\nabla}_{v} f=B\left(v_{3} \underline{e}_{2}-v_{2} \underline{e}_{3}\right) \cdot\left(\frac{\partial f}{\partial v_{1}} \underline{e}_{1}+\frac{\partial f}{\partial v_{2}} \underline{e}_{2}+\frac{\partial f}{\partial v_{3}} \underline{e}_{3}\right)=v_{3} \frac{\partial f}{\partial v_{2}}-v_{2} \frac{\partial f}{\partial v_{3}} \cdot$

Now, by assumption,

$$
\frac{\partial f(\underline{v})}{\partial \phi}=\frac{\partial f\left(v_{1}=v \cos \theta, v_{2}=v \sin \theta \cos \phi, v_{3}=v \sin \theta \sin \phi\right)}{\partial \phi}=0,
$$

which means

$$
\frac{\partial f(\underline{v})}{\partial \phi}=\frac{\partial f}{\partial v_{2}} v \sin \theta(-\sin \phi)+\frac{\partial f}{\partial v_{3}} v \sin \theta \cos \phi=-v_{3} \frac{\partial f}{\partial v_{2}}+v_{2} \frac{\partial f}{\partial v_{3}},
$$

and therefore

$$
\underline{\mathrm{v}} \times \underline{\mathrm{B}} \cdot \underline{\nabla}_{\mathrm{v}} f=-\frac{\partial f}{\partial \phi}=0 \text { 。 }
$$

\section{APPENDIX A-2,}

Derivation of the relationships between the actual time $t$ and the various T:'s of the initial-distribution (i.e., no: source) type calculation.

By (31), (A-2.1),

$$
\tau=\Gamma \cdot A t,
$$

where

$$
A=n_{0} / \int_{0}^{\infty} \int_{-\mu_{R}}^{\mu} \int_{0}^{2 \pi} F_{0}(\mu, v) v^{2} d \phi d \mu d v=n_{0} / 2 \cdot 2 \pi v_{0}^{3} \int_{0}^{\infty} \int_{-\mu_{R}}^{\mu_{R}} F_{0}(\mu, x) x^{2} d \mu d x
$$


and where $\Gamma$ was defined in. Eqs. (21) to be

$$
\Gamma \equiv 4 \pi e^{4} \ln \mathrm{D} / \mathrm{m}^{2} \text {. }
$$

D was defined by Eq. (18) as

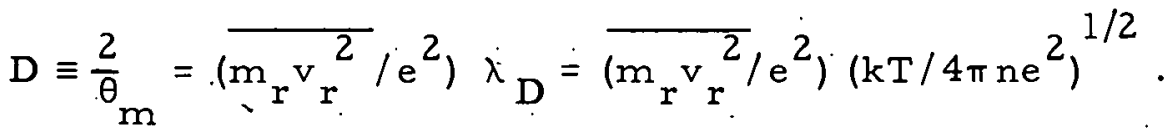

$(18),(\mathrm{A}-2.4)$

Because $D$ is a very large number, in $D$ is quite insensitive to relatively small errors in the evaluation of $D$. Hence, $\overline{\mathrm{m}_{\mathrm{r}} \mathrm{v}_{\mathrm{r}}^{2}}$ is set equal to $3 \mathrm{kT}$, $\operatorname{and} \mathrm{kT}$ in turn is set equal to the energy of the plasma electrons, $E_{e}$. Thus $\mathrm{D}$ becomes

$$
\mathrm{D}=4.90 \times 10^{14} \mathrm{E}_{\mathrm{e}}^{3 / 2} \mathrm{n}^{-1 / 2}
$$

where $E_{e}$ is in kilovolts and $\mathrm{n}$ is in particles $/ \mathrm{cm}^{3}$; and $\Gamma$ becomes, on changing the logarithm to the base ten,

$$
\begin{aligned}
\Gamma & =\frac{4 \pi e^{4}}{m^{2}} \times 2.30 \log D=1.374 \times 10^{11} \log D \\
& =1.374 \times 10^{11}\left(14.69+\log \frac{E^{3 / 2}}{n^{1 / 2}}\right) \\
& =\left(2.02+0.1374 \log \frac{E^{3 / 2}}{n^{1 / 2}}\right) \times 10^{12} \\
& =2.02 \times 10^{12}\left(1.000+0.1019 \log E_{e}-0.0340 \ldots \log \ldots n\right) .
\end{aligned}
$$

The particle densities usually considered for mirror machines range from about $10^{11}$ to $10^{14} \mathrm{~cm}^{-3}$, centering perhaps around $10^{13} \mathrm{~cm}^{-3}$, and the deuteron energies usually considered range from: about 10 to $10^{3} \mathrm{kev}$. For reasons involving the transfer of energy, the mean electron energies will probably range from about one to one hundred kilovolts. For a particle density of $10^{13}$ 
$\mathrm{cm}^{-3}$ and an electron ene rgy of one kilovolt, $\Gamma=1.127 \times 10^{12}$ : For a particle density of $10^{13} \mathrm{~cm}^{-3}$ and an electron energy of one hundred kilovolts, $\dot{\Gamma}=1.540 \times 10^{12}$.

In evaluating $A$ one notes that

$$
\begin{aligned}
v_{0} & =\sqrt{\frac{2 E}{m}}=\sqrt{\frac{2 \times 6.02 \times 10^{23} \times 1.60 \times 10^{-9}}{2.015}} \mathrm{E}^{1 / 2} \\
& =3.09 \times 10^{7} \mathrm{E}^{1 / 2}
\end{aligned}
$$

where $\mathrm{E}$ is the deuteron energy in kilovolts. Consequently,

$$
A=\frac{2.69 \times 10^{-24}}{\int_{0}^{\infty} \int_{0}^{\mu} R F_{0}(\mu, x) x^{2} d \mu d x} \cdot \frac{n_{0}}{E^{3 / 2}} \cdot
$$

Various values of $A$ arise from the various $F_{0}$ 's considered. These A's are evaluated below. In all cases (except those with a source function for which $\left.F_{0}=0\right) F_{0}$ is of the form $F_{0}(\mu, x)=M_{0}(x) S_{0}(\mu)$. In all cases $M_{0}(x)=e^{-10(x-1)^{2}}:$ In the peaked-in-angle case $S_{0}(\mu)=e^{-100 \mu^{2}}$. In the normal mode case where $R=3.3, \lambda=2$,

$$
S_{0}(\mu)=(-1) Q_{1}(\mu)=1-\frac{\mu}{2} \ln \frac{1+\mu}{1-\mu} .
$$

For the other normal mode cases,

$$
S_{0}(\mu)=\dot{a}\left[1-e^{-\beta\left(\mu_{R}-|\mu|\right)}\right],
$$

where $a:-\frac{1}{1-e^{-\beta \mu_{R}}}$

and

$$
\beta=\frac{2 \mu_{R}}{1 \cdots \mu_{R}^{2}}=2 \sqrt{R(R-1)} .
$$


For $R=10, \lambda=1$,

$$
S_{0}(\mu)=1-e^{-18.96(0.948-|\mu|)} .
$$

And for $R=100, \lambda=0.5$,

$$
S_{0}(\mu)=1-e^{-198(0.995-|\mu|)} \text {. }
$$

The integral in the denominator of Eq. (A-2.8) then becomes

$$
\int_{0}^{\infty} \int_{0}^{\mu_{R}} F_{0}(\mu, x) x^{2} d \mu d x=\int_{0}^{\infty} e^{-10(x-1)^{2}} x^{2} d x \cdot \int_{0}^{\mu_{R}} s_{0}(\mu) d \mu .
$$

For all cases,

$$
\int_{0}^{\infty} e^{-10(x-1)^{2}} x^{2} d x=0.588
$$

For the peaked-in-angle case,

$$
\int_{0}^{\mu_{R}} s_{0}(\mu) d \mu=0.0886 \text {. }
$$

For the normal mode case $R=3.3, \lambda=2$,

$$
\int_{0}^{\mu} S_{0}(\mu) d \mu=0.601
$$

For the normal mode case $R=10, \lambda=1$,

$$
\int_{0}^{\mu} S_{0}(\mu) d \mu=0.896
$$

and for the normal mode case $\mathrm{R}=100, \lambda=0.5$,

$$
\int_{0}^{\mu} S_{0}(\mu) d_{\mu}=0.990 \text {. }
$$

By combining Eqs. A-2. 1; A-2.6, A-2.8, and A-2. 15 through A-2. 19, : we obtain the following conversion factors from $T$ to time, $t$ :

"In combining these equations $\mathrm{n}$ was set equal to $\mathrm{n}_{0^{\circ}}$. This is not quite true, of course, and $\Gamma$ changes slowly with time. However, for a loss of $90 \%$ of the initial plasma, 1 would change by only $3 \%$. 
For the peaked-in-angle case,

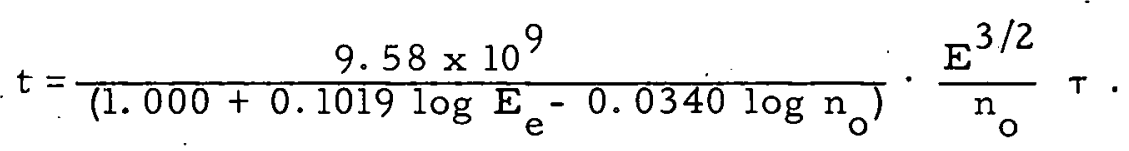

For the normal mode, $\mathrm{R}=3.3, \lambda=2$,

$$
t=\frac{6.51 \times 10^{10}}{\left(1.000+0.1019 \log E_{e}-0.0340 \log n_{0}\right)} \cdot \frac{E^{3 / 2}}{n_{o}} \tau \text {. }
$$

For the normal modc, $\mathrm{R} \equiv 10, \lambda=1$,

$$
\mathrm{t}=\frac{9.71 \times 10^{10}}{\left(1.000+0.1019 \log \mathrm{E}_{\mathrm{e}}-0.0340 \log \mathrm{n}_{\mathrm{o}}\right)^{-0}} \cdot \frac{\mathrm{E}^{3 / 2}}{\mathrm{n}_{\mathrm{o}}} \tau,
$$

and for the normal mode, $R=100, \lambda=0.5$,

$$
\mathrm{t}=\frac{1.071 \times 10^{11}}{\left(1.000+0.1019 \log E_{e}-0.0340 \log n_{0}\right)} \cdot \frac{E^{3 / 2}}{n_{o}} \tau \text {. }
$$

In all the above equations, $t$ is in seconds, $E$ and $E$ are in kilovolts, and $n_{0}$ is in particies $/ \mathrm{cm}^{3}$.

For ease in comparing the various results given in Part A of this report, the above $\tau^{\prime}$ 's have been adjusted so that in all cases the results are tahula.ter or plotted ateuming

$$
t=\frac{1.000 \times 10^{10}}{\left(1.000+0.1019 \log E_{e}-0.0340 \operatorname{lng} n_{0}\right)} \cdot \frac{E^{3 / 2}}{n_{o}} \tau .
$$

Equation (A-2.24) applies only to the results presented in Part A. The $\tau$ 's of Part B are those of Eqs. (A-2.20) and (A-2.21).

For $\mathrm{E}=100 \mathrm{kev}, \mathrm{E}_{\mathrm{e}}=1 \mathrm{kev}$, and $\mathrm{n}_{\mathrm{u}}=10^{13} \mathrm{particles} / \mathrm{cm}^{3}, \mathrm{Eq}_{\mathrm{q}} \cdot(\Lambda-2.2 .4)$ indicates that $t$ in seconds is equal to $1.792 \tau$.

\section{APPENDIX A-3}

Derivation of expressions connecting the time $t$ with the time parameter $\tau_{s}$, and the particle density with the distribution function, for the separated solutions with a source function. 
In the calculations made with Eq. (51),

$$
\left(\frac{\partial M}{\partial T_{s}}\right)_{C}=4 \pi M^{2}+\frac{1}{2} G_{x x} M_{x x}+\frac{1}{x^{2}} G_{x} M_{x}-\frac{G_{x}}{2 x^{3}} \lambda M+K\left(x, \tau_{s}\right) \text {, }
$$

where

$$
\tau_{\mathrm{S}}=\Gamma \mathrm{At}
$$

the normalizing factor A was connected to the injection current density.

Since the initial particle density was presumed to be zero in the calculations with a source, A naturally could not be related to an initial particle density. However, as before, A does connect the particle density $n$ with the distribution function used in the calculation:

$$
\begin{aligned}
\mathrm{n} & =\int_{0}^{\infty} \int_{-\mu_{R}}^{\mu} \int_{0}^{2 \pi} f(\mu, v) v^{2} \mathrm{dvd} \mu \mathrm{d} \phi \\
& =\mathrm{A} \int_{0}^{\infty} \int_{-\mu_{R}}^{\mu} \int_{0}^{2 \pi} F(\mu, v) v^{2} \mathrm{dvd} \mu \mathrm{d} \phi \\
& =A \cdot 4 \pi v_{0}^{3} \int_{0}^{\infty} M(x) x^{2} d x \int_{0}^{\mu} R S(\mu) \mathrm{d} \mu .
\end{aligned}
$$

The source function $K\left(x, \tau_{s}\right)$ was also defined to give the injection current density through a comection with $\Lambda$ :

$$
\begin{aligned}
& \left.\frac{j(\text { particles }}{\mathrm{cm}^{3} \tau}\right)=A \cdot 4 \pi v_{0}^{3} \int_{0}^{\infty} K(x) x^{2} d x \int_{0}^{\mu} R S(\mu) d \mu, \\
& J\left(\frac{\text { particles }}{\mathrm{cm}^{3} \mathrm{sec}}\right)=\Gamma A j=A^{2} \cdot 4 \pi v_{0}^{3} \Gamma \int_{0}^{\infty} K(x) x^{2} d x \int_{0}^{\mu R} S(\mu) d \mu .
\end{aligned}
$$

In this calculation the source function $\mathrm{K}$ was taken to be constant in time and a $\delta$-function in speed:

$$
K(x, t)=K(x)=K_{0} \delta(x-1)
$$


so that

$$
\int_{0}^{\infty} K(x) x^{2} d x=\int_{0}^{\infty} K_{0} \delta(x-1) x^{2} d x=K_{0}
$$

Substitution of (A-3.7) into (A-3.5) led to

$$
\mathrm{K}_{\mathrm{o}}=\frac{\mathrm{J}}{\mathrm{A}^{2} \cdot 4 \pi \mathrm{v}_{0}^{3} \Gamma \int_{0}^{\mu} \mathrm{R} S(\mu) \mathrm{d} \mu} \quad \cdots \quad \text { (A-3.8) }
$$

$\mathrm{K}_{\mathrm{O}}$ was chosen rather arbitrarily to be $10^{-4}$. Choosing $\mathrm{K}_{\mathrm{n}}$ merely scaled the size of the $M(x)$ 's to be dealt with in the calculation.

The normallzing factor $A$ was evaluated from (A-3. 8):

$$
A=\left(\frac{J}{4 \pi v_{0}^{3} \Gamma \int_{0}^{\mu} R S(\mu) d \mu \cdot 10^{=4}}\right)^{1 / 2} \text {. }
$$

Substitution of (A-3.9), (A-2.6), and (A-2.7) into (A-3.2), noting from (A-2. 18), that $\int_{0}^{\mu} R(\mu) d \mu=0.896$ for $R=10$, and similarly that $\int_{0}^{\mu} R$ 0.466 for $R=2$, gave for $R=2$

$t=2.92 \times 10^{3}\left(\frac{E^{3 / 4}}{J^{1 / 2}}\right) \frac{\tau_{s}}{\left(1.000+0.1019 \log E_{e}-0.0340 \log n\right)^{1 / 2}}, ": \quad(4 \dot{y}),(A-3.1 U)$

and for $R=10$

$t=4.05 \times 10^{3}\left(\frac{E^{3 / 4}}{J^{1 / 2}}\right) \frac{T_{s}}{\left(1.000+0.1010 \log E_{e}-0.0340 \log 11\right)^{1 / 2}}$.

Making the above substitutions in (A-3.3) gave: for $R=2$, $n(t)=2.92 \times 10^{7} \frac{E^{3 / 4} J^{1 / 2}}{\left(1.000+0.1019 \log E_{\left.e^{-0.0340 ~} \log n\right)^{1 / 2}}\right.} \int_{0}^{\omega} M(x, t) x^{2} d x, \quad(A-3.12)$ a.n. for $R=10$

$$
n(t)=4.05 \times 10^{7} \frac{E^{3 / 4} J^{1 / 2}}{\left(1.000+0.1019 \log E_{e}-0.0340 \log n\right)^{1 / 2}} \int_{0}^{\infty} M(x, t) x^{2} d x .
$$


In the discussion of the initial-distribution-type separated solutions, it was pointed out that the apparent particle loss-rate constants were about twice as big as the loss-rate constants of the unseparated solutions. Application of the implied correction of approximately $\sqrt{2}$ to the particle densities gave, for $\mathrm{R}=2$,

$n(t)=4.13 \times 10^{7} \frac{E^{3 / 4} J^{1 / 2}}{\left(1.000+0.1019 \log E_{e}-0.0340 \log n\right)^{1 / 2}} \int_{0}^{\infty} M(x, t) x^{2} d x$

and for $R=10$,

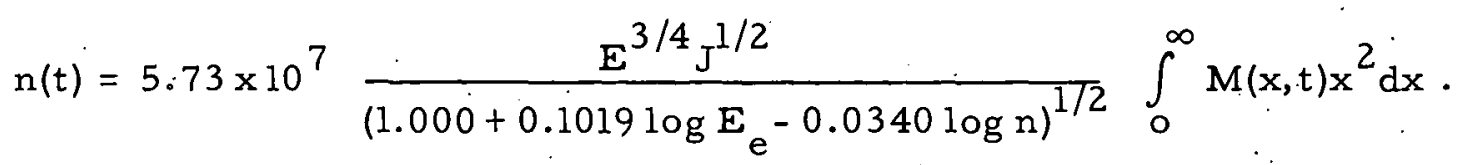

/ nir 


\section{B - COMPUTATIONAL PART}

This discussion will be restricted to the unseparated solutions of Eq. (27) of Part A because they presented the much greater number of computational dificulties. Also, any difficulties associated with the simpler separated solutions tended to carry over into the more complicated approach.

The basic equation, Eq. (27) of Part A, had the form:

$$
\begin{aligned}
\frac{\partial F}{\partial q} & =4 \pi F^{2}+\frac{1}{2} G_{x x} F_{x x}+\left(\frac{\left(1-\mu^{2}\right)}{x^{2}} G_{\mu x}-\frac{\left(1-\mu^{2}\right)}{x^{3}} G_{\mu}\right) F_{\mu x} \\
& +\left(\frac{\left(1-\mu^{2}\right)}{2 x^{4}} G_{\mu \mu}+\frac{\left(1-\mu^{2}\right)}{2 x^{3}} G_{x}-\frac{\mu\left(1-\mu^{2}\right)}{2 x^{4}} G_{\mu}\right) F_{\mu \mu} \\
& +\left(\frac{\left(1-\mu^{2}\right)}{2 x^{3}} G_{\mu \mu}+\frac{1}{x^{2}} G_{x}-\frac{\mu}{x^{3}} G_{\mu}\right) F_{x} \\
& +\left(-\frac{\left(1-\mu^{2}\right)}{x^{3}} G_{\mu x}-\frac{\mu\left(1-\mu^{2}\right)}{2 x^{4}} G_{\mu \mu}-\frac{\mu}{x^{3}} G_{x}+\frac{1}{x^{4}} G_{\mu}\right) F_{\mu} .
\end{aligned}
$$

where

$$
G(\mu ; x)=\int_{0}^{\infty} \int_{-\mu_{R}}^{\mu_{R}} \int_{0}^{2 \pi} x^{\prime 2} F\left(\mu^{\prime}, x^{\prime}\right) x_{r} d \psi d \mu^{\prime} d x^{\prime},
$$

and

$$
x_{r}=\left[x^{2}+x^{\prime 2}-2 x x^{\prime}\left(\mu \mu !+\sqrt{1-\mu^{2}} \sqrt{1-\mu^{\prime 2}}\right) \cos \phi\right]^{1 / 2} .
$$

As shown in Appendix $B-1, G(\mu, x)$ may be transformed to

$$
G(\mu, x)=4 \int_{0}^{\infty} \int_{-\mu_{R}}^{\mu} x^{\prime}{ }^{2} F\left(\mu^{\prime}, x^{\prime}\right) q^{1 / 2} E(k) d \mu^{\prime} d x^{\prime},
$$

where $E(k)$ is the complete elliptic integral of the second kind,

$$
\begin{aligned}
& k=\sqrt{p / q}, \quad p=4 x x^{\prime} \sqrt{1-\mu^{2}} \sqrt{1-\mu^{\prime 2}}, \quad \text { and } \\
& q=x^{2}+x^{\prime 2}-2 x x^{\prime}\left(\mu \mu^{\prime}-\sqrt{1-\mu^{2}} \sqrt{1-\mu^{\prime 2}}\right) .
\end{aligned}
$$


$F$ is subject to the conditions:

$$
\begin{aligned}
& 0 \leqslant F(\mu, x) \leqslant 1, \\
& F(\mu, x)=0 \text { for } \mu=\mu_{R^{\prime}} \\
& F_{(\mu=0, x=1)}^{T=0}=1, \text { and } \\
& F(\mu, x)=F(-\mu, x)
\end{aligned}
$$

\section{NUMERICAL METHODS USED}

The partial differential equation was solved nume rically by converting it to an explicit centered difference equation. The factoring done here was only for convenience.

$$
\begin{aligned}
& \frac{F(\mu, x)-F^{\top}(\mu, x)}{\Delta T}=\frac{1 .}{2 x^{4}}:\left\{\pi x^{4}\left(F^{\top}(\mu, x)\right)^{2}+\bar{A}\left(\frac{F^{\top}(\mu, x+1)-2 F^{\top}(\mu, x)+F^{\top}(\mu, x-1)}{[\Delta x]^{2}}\right)\right. \\
& +\bar{B}\left(\frac{F^{\top}(\mu+1, x+1)-F^{\top}(\mu-1, x+1)-F^{\top}(\mu+1, x-1)+F^{\top}(\mu-1, x-1)}{4 \Delta \mu \Delta x}\right) \\
& +\bar{C}\left(\frac{F^{\top}(\mu+1, x)-2 F^{\tau}(\mu, x)+F^{\top}(\mu-1, x)}{[\Delta \mu]^{2}}\right) \\
& \left.+\bar{D}\left(\frac{F^{\top}(\mu, x+1)-F^{\top}(\mu, x-1)}{2 \Delta x}\right)+\bar{E}\left(\frac{F^{\top}(\mu+1, x)-F^{\top}(\mu-1, x)}{2 \Delta \mu}\right)\right\} \text {, }
\end{aligned}
$$

where $\bar{A}=x^{4} \cdot{ }_{x x}$,

$$
\begin{aligned}
& \bar{B}=2 x\left(1-\mu^{2}\right)\left[x G_{\mu x}-G_{\mu}\right], \\
& \bar{C}=\left(1-\mu^{2}\right)\left[G_{\mu \mu}+x G_{x}-\mu G_{\mu}\right], \\
& \bar{D}=x\left[\left(1-\mu^{2}\right) G_{\mu \mu}+2 x G_{x}-2 \mu r_{\mu}\right],
\end{aligned}
$$




$$
\bar{E}=-2 x\left(1-\mu^{2}\right) G_{\mu x}-\mu\left(1-\mu^{2}\right) G_{\mu \mu}-2 \mu x G_{x}+2 G_{\mu},
$$

and

$$
\begin{aligned}
& G_{\mu}=\left[G^{\top}(\mu+1, x)-G^{\top}(\mu-1, x)\right] / 2 \Delta_{\mu} \\
& G_{x}=\left[G^{\top}(\mu, x+1)-G^{\top}(\mu, x-1)\right] / 2 \Delta x \\
& G_{\mu \mu}=\left[G^{\top}(\mu+1, x)-2 G^{\top}(\mu, x)+G^{\top}(\mu-1, x)\right] /(\Delta \mu)^{2} \\
& G_{x x}=\left[G^{\top}(\mu, x+1)-2 G^{\top}(\mu, x)+G^{\top}(\mu, x-1)\right] /(\Delta x)^{2} \\
& G_{\mu x}=\left[G^{\top}(\mu+1, x+1)-G^{\top}(\mu-1, x+1)-G^{\top}(\mu+1, x-1)+G^{\top}(\mu-1, x-1)\right] / 4 \Delta \mu \Delta x .
\end{aligned}
$$

The $G(\mu, x)$ integral was calculated numerically by Weddle's rule for inte:gration. (This is a quadrature formula which may be found in any standard nume rical analysis reference.)

$G^{\top}(\mu, x)=\left(\frac{3}{10} \Delta x^{\prime}\right)\left(\frac{3}{10} \Delta_{\mu^{\prime}}\right) \cdot 4 \sum_{x^{\prime}=0}^{x_{\max }}\left(w_{j}\right) x_{j}^{\prime}{ }_{j} \sum_{\mu^{\top}=-\mu_{R}}^{+\mu_{i}}\left(w_{i}\right) F\left(\mu_{i}^{\prime}, x_{j}^{\prime}\right) q^{1 / 2} E(k)$

where

$$
k=\sqrt{p / q} .
$$

The complete elliptic integral, $E(k)$, was solved by use of Landen's trans formation. [See Hancock, Harris, Elliptic Integrals (Dover Publ. Inc., N. Y., 1958)\%]

where

$$
E\left(k_{0}\right)=\frac{\pi}{2}\left[\prod\left(1+K_{i}\right)\right] \cdot\left[1-\frac{k_{0}^{2}}{2}\left(1+\frac{K_{1}}{2}+\frac{K_{1} \cdot K_{2}}{2 \cdot 2}+\frac{K_{1} \cdot K_{2} \cdot K_{3}}{2 \cdot 2 \cdot 2}+\ldots\right],\right.
$$

$$
\mathrm{K}_{\mathrm{i}}=\left(1-\sqrt{1-\mathrm{k}_{\mathrm{i}}^{2}}\right) /\left(1+\sqrt{1+\mathrm{k}_{\mathrm{i}}^{2}}\right) \text { and } \mathrm{k}_{i+1}^{2}=\mathrm{K}_{\mathrm{i}}^{2} \text {. }
$$

This was calculated on the machine by "nesting ":

$$
E\left(k_{0}\right)=\frac{\pi}{2}\left[\prod\left(1+K_{i}\right)\right] \cdot\left[1-\frac{k_{0}^{2}}{2}\left(1+\frac{K_{1}}{2}\left(1+\frac{K_{2}}{2}\left(1+\frac{K_{3}}{2}(\ldots)\right)\right)\right)\right] .
$$




\section{BOUNDARY CONDITIONS}

The use of central differences demanded values of $F(\mu, x)$ and $G(\mu, x)$ at $\mu_{0}-\Delta \mu, \quad \mu_{R}+\Delta \mu, \quad x_{0}-\Delta x$, and $x_{\max }+\Delta x$, where $x_{0}$ represents $x=0$ and $\mu_{0}$ reprësents $\mu=0$.

Since the basic equations were symmetric with respect to $\mu$, the boundary c onditions at $\mu_{0}$.were immediately apparent.

At $\mu_{0}, F\left(\mu_{0}-\Delta \mu, x\right)=F\left(\mu_{0}+\Delta \mu, x\right)$ and $G\left(\mu_{0}-\Delta \mu, x\right)=G\left(\mu_{0}+\Delta \mu, x\right)$.

At $\mu_{R}, F\left(\mu_{R}, x\right)=0$, and therefore no problems occurred at these points. $F\left(\mu_{R}, x\right)$ was constant and $F\left(\mu_{R}-\Delta \mu, x\right)$ could be easily calculated using $F\left(\mu_{R}, x\right)$ and $G\left(\mu_{R}, x\right)$. Note that $G(\mu, x)$ did not depend upon central differences and could be calculated for any point at which an. $F(\mu, x)$ existed. In the $\mu_{0}-\Delta \mu$ case, the $G(\mu, x)$ already existed and its calculation would have been superfluous.

Similar methods were employed at the $x_{0}$ and $x_{\max }$ boundaries. $F\left(\mu, x_{0}\right)$ and $F\left(\mu, x_{\max }\right)$ were not calculated by the partial differential equation, therefore making $F\left(\mu, x_{0}+\Delta x\right)$ and $F\left(\mu, \dot{x}_{\max }-\Delta x\right)$ the limits to which the basic equation was effective.

For physical reasons, discussed in Part $A, F\left(\mu, x_{0}\right)$ was set equal to zero for a.11 times. Therefore

$$
\text { at } x=0, \quad F\left(\mu, x_{0}\right)=F^{\top}\left(\mu, x_{0}\right)
$$

At $x_{\max }$ an attempt was made to simulate roughly the changes occurring in nearby $F(\mu, x)$ 's. $F\left(\mu, x_{\text {max. }}\right)$ was allowed to change in proportion to $F\left(\mu, x_{\text {max }}-\Delta x\right)$.

$$
\text { At } x_{\text {max }}, F\left(\mu, x_{\text {max }}\right)=\frac{F^{\top}\left(\mu, x_{\text {max }}\right)}{F^{\top}\left(\mu, x_{\text {max }}-\Delta x\right)} \cdot F\left(\mu, x_{\text {max }}-\Delta x\right)
$$

This $x_{\max }$ boundary condition suffyed when the initial distribution functions 
were not sharply exponential near $x_{\max }$

\section{STABILITY CONDITIONS}

The rapidity of advancement of $F(\mu, x)$ with respect to time was subject to the most rigid of the following restrictions on $\Delta \tau$ :

$\left.\Delta_{T}<\left|\frac{x^{4}(\Delta x)^{2}}{\bar{A}}\right|, \Delta_{T}<\left|\frac{4 x^{4} \Delta \mu \Delta x}{\bar{B}}\right|, \Delta T<\left|\frac{x^{4}(\Delta \mu)^{2}}{\bar{C}}\right|, \quad \Delta T<\mid \frac{4 x^{4} \Delta x}{\bar{D}}\right\rceil$, and $\quad \Delta \tau<\left|\frac{4 x^{4} \Delta \mu}{\bar{E}}\right|$.

The terms involving $\bar{A}, \bar{B}$, and $\bar{C}$ proved to be the most stringent of these conditions.

Since $G(\mu, x)$ assumed its lowest values at small values of $\mu$ and $x$, the dominating restrictions occurred at $x=x_{0}+\Delta x$.

In actual practice these stability conditions were not sufficiently rigorous, but provided a useful estimate: As an example, for one problem these equations yielded $\Delta_{i} T<0.00045$. Instability occurred during trial runs using $\Delta T=0.0004$ and $\Delta_{T}=0.0002$, but by using $\Delta_{T}=0.0001$ there was no trouble. This instability can be easily recognized by erratic behavior of the $F(\mu, x)$ grid in the $\mu$ direction at small values of $x$.

\section{MACHINE CALCULATION}

The difference equations were coded for an IBM 704, with an 8192-word memory.

Special efforts were necessary when coding the numerical integration of the $G(\mu, x)$ integral because of its time-consuming nature. The equation was broken down to avoid as muchirecalculation as possible. 
where

$$
G(\mu, x)=\sum_{x_{0}}^{x_{\max }} \sum_{\mu_{0}}^{\mu_{R}} F\left(\mu_{i}^{\prime}, x_{j}^{\prime}\right) \Lambda_{i j}
$$

$$
\Lambda_{i j}=4 \cdot \frac{3}{10} \Delta x^{\prime} \cdot \frac{3}{10} \Delta \mu^{\prime} w_{j} x_{j}^{\prime 2} w_{i} \cdot q_{i, j}{ }^{1 / 2} E(k)_{i, j}
$$

(in $\Lambda_{i j}$ the negative $\mu$ 's are included).

For every point $\left(\mu_{i}, x_{j}\right)$ there are $(\#(+\mu) \cdot \# x) \Lambda_{i j}$ 's. "Note that $\Lambda_{i j}$ is not a function-of $\tau$. These numerous $\Lambda_{\mathrm{ij}}$ 's were calculated once for a given set of $(\Delta \mu, \Delta \mathrm{x}, \# \mu, \# \mathbf{x})^{\prime \prime}$ and stored on tapes. A complete $G(\mu, x)$ grid could then be calculated at any time during a problem in only 1 to $1 \frac{1}{2}$ minutes, depending upon the size of the grid.

When the equations had been completely coded and were applied to specific problems it became apparent that $G(\mu, x)$ need not be calculated at every time step. When $\Delta \mathrm{x}$ and $\Delta \mu$ were chosen to be approximately 0.1 , stability conditions allowed only small values of $\Delta_{T}$ and the refore the $F(\mu, x)$ grid advanced very slowly with respect to $\tau \cdot G(\mu, x)$ was not very sensitive to changes in $F(\mu, x)$, especially when $F(\mu, x)$ changed slowly.

After the method of $G(\mu, x)$ calculation was determine $d$, the $F(\mu, x)$ equation presented no special coding problems. For machine computational purposes the form of the $\Gamma(\mu, x)$ equation wao exractly as follows:

$$
\begin{aligned}
F(\mu, x) & =F^{\top}(\mu, x)+\frac{\Delta T}{2 x^{4}}\left[8 \pi x^{4}\left(F^{\top}(\mu, x)\right)^{2}-2\left(\frac{\bar{A}}{(\Delta x)^{2}}-\frac{C}{(\Delta \mu)^{2}}\right) F^{\top}(\mu, x)\right. \\
& +\left(\frac{\bar{A}}{(\Delta x)^{2}}+\frac{\bar{D}}{2 \Delta x}\right) F^{\top}(\mu, x+1)+\left(\frac{\bar{A}}{(\Delta x)^{2}}-\frac{\bar{D}}{2 \Delta x}\right) F^{\top}(\mu, x-1) \\
& +\left(\frac{\bar{C}}{(\Delta \mu)^{2}}+\frac{\bar{E}}{2 \Delta \mu}\right) F^{\top}(\mu+1, x)+\left(\frac{\bar{C}}{(\Delta \mu)^{2}}-\frac{\bar{E}}{2 \Delta \mu}\right) F^{\top}(\mu-1, x) \\
& \left.+\frac{\bar{B}}{4 \Delta \mu \Delta x} \cdot\left(F^{\top}(\mu+1, x+1)-F^{\top}(\mu-1, x+1)-F^{\top}(\mu+1, x-1)+F^{\top}(\mu-1, x-1)\right)\right]
\end{aligned}
$$

*\#(+ $\mu$ ) designates the number of values of positive $\mu$ included in the computation and \#x designates the number of different $x$ values included in the computation. 
FLOW DIAGRAM OF ALICE

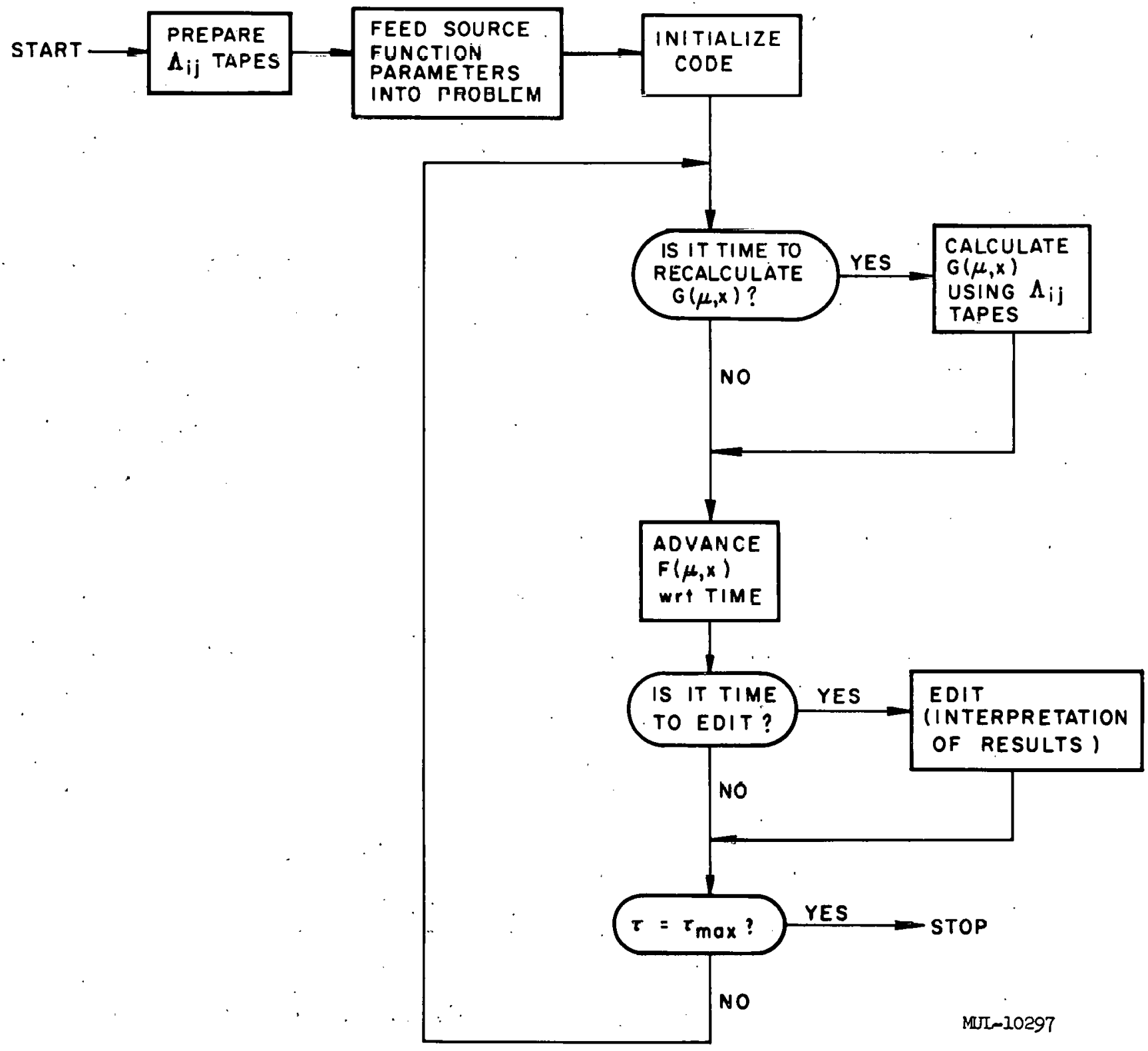

Flow Diagram of Allce. 
$F(\mu, x)$ at $T=0$ was calculated manually since the initial function $F(\mu, x)=$ $M(x) S(\eta j)$ varied with' each problem. Other variables were $\Delta \mu, \Delta x$, and \#x. The \# $\mu$ was restricted to 10 angles from 0 to $\mu_{R}$. Since the equations were symmetric with respect to $\mu$, this meant 19 positive and negative angles. The maximum \#x permitted was 49.

As the $F(\mu, x)$ grid slowly advanced with respect to $\tau$, the peak of the distribution function flattened out and $\int_{0}^{x} \int_{0}^{\mu} R(\mu, x) x^{2} d \mu d x$ diminished. The values of $G(\mu, x)$ became smaller, the values of $\bar{A}, \bar{B}, \bar{C}, \bar{D}$, and $\bar{E}$ became smaller, and the stability conditions permitted a larger time interval. For this reason the stability conditions were incorporated into the code and $\Delta T$ was allowed to increase as soon as possible. Only the 3 most stringent conditions were used in the code-those involving $\dot{\bar{A}}, \overline{\mathrm{B}}$, and $\overline{\mathrm{C}}$.

In checking for machine errors we relied primarily upon looking at the edited output. A test in the code for negative values of $F(\mu, x)$ was désigned to find obvious errors.

The edited output of $T$ consisted of supplementary, integrals based upon the distribution function, and the actual $F(\mu, x)$ grid:

\section{SPECIAL BOUNDARY CONDITIONS USED FOR A}

\section{COARSE GRID}

A special problem arose when we used a peaked-in-angle initial distribution function, $F(\mu, x)=e^{-10(x-1)^{2}} \cdot e^{-100 \mu^{2}}$. At large values of $\mu$ and $x$ the $F(\mu, x)$ grid was exceedingly coarse. When this grid.(i.e., this two-dimensional table of $F$ valies) was given to the code, it was discovered that not even qualitative results could be obtained in this region. The equations thoughtfully chewed these coarse values or a time, digested them and then informed us that the $F(\mu, x)$ 's 
at these points were getting smaller. These $F(\mu, x)$ 's continued to decrease until: they could no longer be expressed as floating point numbers and had to be set equal to zero. As the $F(\mu, x)$ grid was advanced with respect to $T$ it became apparent that the grid did not wish to merely approach zero in the re'gion $\mu_{3}$ to $\mu_{8}$ and $x_{16}$ to $x_{\text {max }}$ but rather it desired to be zero in this region. Hand calculations showed that $\bar{B} F_{\mu x}$, which was negative at these points, was making the major contributions to $\Delta \bar{F} \cdot \bar{B} F_{\mu x}$ was the only term in which $F(\mu-1, x-1)$ occurred. Wherever. $F(\mu-1, x-1)$ was considerably larger than $F(\mu, x)$ and its neighbors, $\Delta F(\mu, x)$ was negative.

Since such loss of particles at large $\mu$ and large $x$ was physically unrealistic, it was decided to use special zero boundary conditions to force the grid to simulate the physical model. Then the major part of the grid, which was not so coarse, could be calculated normally.

The special boundary conditions were applicable in two different cases. They helped to prevent zeros and they promoted growth in zero regions. $\tau+\Delta \tau$ $T+\Delta T$

If the equations produccd an $F(\mu ; x)=0$ whcn $F^{\top}(\mu, x)$ had not been zero, $F(\mu, x)$ was made proportional to its neighbors in the same way that $F^{\top}(\mu, x)$ had been. This prevented zeros in most instances. $T+\Delta T$

If $\mathrm{F}^{\top}(\mu, \mathrm{x})$ had been zero, the grid was encouraged to grow by calculating $F(\mu, x)$ proportional to its neighbors. This $F(\mu, x)$ was usually zero also, but occasionally it would extend the nonzero part of the grid.

These zero boundary condtions forced growth at zero boundaries contrary to the actions of the $F(\mu, x)$ equations, and consequently perturbations occurred which made the results erroneous in this region. However, the magnitude of the F's, erroneous or not, was so small.in this region as to be essentially zero for all physical purposes. Also, comparison with a rough fixed-point calculation showed that this region had little or no effect on the main part 
of the grid, that is, the less-radically exponential part, and that our results were reasonable.

\section{SUPPLEMENTARY CALCULATIONS}

The preceding sections were devoted to advancing the $F(\mu, x)$ grid with respect to time and resulted only in new grids $F\left(\mu, x^{+}\right)^{\top}$. The following sections concern supplementary calculations based upon the new grids. The calculations were performed with every edit.

Weddle's rule was used for numerical calculation of almost all of the integrals. The only exceptions were in the estimates of relative particle density between restricted $\mu$ ranges and $x$ ranges. For the integrals over restricted ranges, rough summations were used.

\section{PARTIC.LE DENSITY}

If $n_{R}$ is the relative number of particles remaining in the mirror machine a.t. time ' $T$, then

$$
n_{R}=\frac{n^{\top}}{n^{\top}=0}
$$

where

$$
n^{\top} \equiv 2: 2 \pi \int_{0}^{\infty} \int_{0}^{\mu} R x^{2} F^{\top}(\mu, x) d \mu d x
$$

and

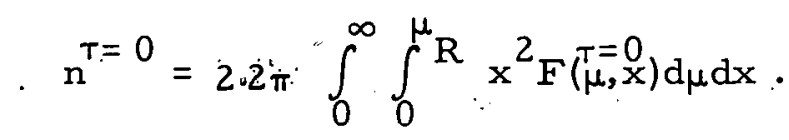

..... In addition, estimates were made of relative particle density between reetricted $\mu$ ranges and between restricted $x$ ranges,

$$
\begin{aligned}
& n_{x_{0}}=\frac{2 \cdot 2 \pi}{N} \int_{x_{0}}^{x_{7}} \int_{\mu_{0}}^{\mu_{R}} x^{2} F^{\top}(\mu, x) d \mu d x \\
& n_{x_{7}}=\frac{2 \cdot 2 \pi}{N} \int_{x_{7}}^{x_{12}} \int_{\mu_{0}}^{\mu_{R}} x^{2} F^{\top}(\mu, x) d \mu d x
\end{aligned}
$$




$$
\begin{aligned}
& \dot{n}_{x_{12}}=\frac{2 \cdot 2 \pi}{N} \int_{x_{12}}^{x_{16}} \int_{\mu_{0}}^{\mu_{R}} x^{2} F^{\top}(\mu, x) d \mu d x \\
& n_{x_{16}}=\frac{2 \cdot 2 \pi}{N} \int_{x_{16}}^{x_{2}} \int_{\mu_{0}}^{\mu_{R}} x^{2} F^{\top}(\mu, x) d \mu d x \\
& n_{x_{22}}=\frac{2 \cdot 2 \pi}{N} \int_{x_{22}}^{x_{\max }} \int_{\mu_{0}}^{\mu_{0}} x^{2} F^{\top}(\mu, x) d \mu d x
\end{aligned}
$$

In lliese equaliuns $\mathrm{IJ}$ is a thurmalizing factur. These were nurmalized in two ways: $N=n^{\tau=0}$ and $N=n^{\top}$.

\section{Mean Energy}

The mean energy was represented by

$$
\overline{x^{2}}=\frac{2 \cdot 2 \pi}{n^{\top}} \int_{0}^{\infty} \int_{\mu_{0}}^{\mu} R x^{2} F^{\top}(\mu, x) \cdot x^{2} d \mu d x .
$$

\section{VII: CIIARGE-EXCIIANGE RATE}

For this calculation the neutral particles were presumed to be cold, i.e., $v_{n} \cong 0$ making $v_{r} \cong v$, so that the $\sigma \mathrm{v}$ for charge exchange could be written as

$$
\overline{\sigma v}=\frac{v_{0}}{N} \cdot \int_{0}^{\infty} \int_{\mu_{0}}^{\mu} R_{x^{2}} F^{\top}(\mu, x) \cdot x \cdot \sigma(x) d \mu d x,
$$

where $\mathrm{N}$ is a normalizing factor. $\overline{\sigma v}$ was normalized in two ways:

$$
\mathrm{N}=\mathrm{n}^{\top=0} \text { and } \mathrm{N}=\mathrm{n}^{\top} \text {. }
$$

The tcrm $\overline{\sigma v}$ wa s calculated at energy levels $1,2,4,8,16,32,63,125$, 250,500 , and 1000 kev.

The $\sigma(x)$ cross sections for these energies at intervals of $\Delta x=0.1$ were stored in 704 memory. Since $\Delta x=0.1$ was chosen for all problems run here, interpolation was never necessary and this calculation consumed a very negligible amount of machine time. 


\section{FUSION REACTION RATE}

This was an exceedingly long computation when reduced to nume rical methods. The other supplementary calculations were easily incorporated into the code of the basic equations, but this was coded separately. Becaúse the code is not dependent upon the previous equations, it seems useful to sketch the development of the se equations.

Fusion reaction rate was defined as

$$
\mathrm{R}=\frac{1}{2} \mathrm{n}^{2} \overline{\sigma \mathrm{v}} \text { for } \mathrm{DD} \text {, and } \mathrm{R}=\frac{1}{4} \mathrm{n}^{2} \overline{\sigma \mathrm{v}} \text { for } 50-50 \mathrm{DT} \text {, }
$$

where $\mathrm{n}$ is the total number of reactant particles.

The values of $\overline{\sigma v}$ were calculated from the equation

$$
\overline{\sigma v}=\frac{\left[2 \pi \int_{0}^{\infty} 2 \int_{0}^{\mu} R v^{2} f(\mu, v) \int_{0}^{\infty} \int_{-\mu}^{\mu} R \int_{0}^{\pi} v^{\prime 2} f\left(\mu^{\prime}, v^{\prime}\right) \cdot v_{r} \sigma\left(v_{r}\right) d \phi d \mu^{\prime} d v^{\prime} d \mu d v\right]}{\left[2 \pi \int_{0}^{\infty} 2 \int_{0}^{\mu} R v^{2} f(\mu, v) d \mu d v\right]^{2}},
$$

where

$$
v_{r}=\left[v^{2}+v^{\prime 2}-2 v v^{\prime}\left(\mu \mu{ }^{\prime}+\sqrt{1-\mu^{2}} \sqrt{1-\mu^{\prime 2}}\right) \cos \phi\right]^{1 / 2} .
$$

The divisor is a normalizing factor causing normalization with respect to the number of particles present.

The substitution $\mathrm{v}=\mathrm{v}_{\mathrm{o}} \mathrm{x}$ was made; where

$$
v_{0}=\sqrt{\frac{2 k T}{M}}=3.10 \times 10^{7} \sqrt{E} \text {. }
$$

By taking $\sigma\left(\mathrm{x}_{\mathrm{r}}^{2}\right) \cdot \mathrm{x}_{\mathrm{r}}$ rather than $\sigma\left(\mathrm{x}_{\mathrm{r}}\right)$ from cross section graphs, a square root calculation was avoided in the most repeated part of the calculation.

$$
v_{r} \sigma\left(v_{r}\right) \equiv a\left(x_{r}^{2}\right) \cdot \frac{v_{o}}{\sqrt{E}}
$$


The in

$\overline{\sigma v}=\frac{\left[2 \pi \cdot \frac{\mathrm{v}}{\sqrt{E}} \int_{0}^{\infty} 2 \int_{\mu_{0}}^{\mu}{ }^{R} x^{2} F(\mu, x) \int_{0}^{\infty} \int_{-\mu_{R}}^{\mu} R \int_{0}^{\pi} x^{\prime 2} F\left(\mu, x^{\prime}\right) a\left(x_{r}{ }^{2}\right) d \phi d \mu^{\prime} d x^{\prime} d \mu d x^{\prime}\right]}{\left[2 \pi \int_{0}^{\infty} 2 \int_{\mu_{0}}^{\mu} x^{2} F(\mu, x) d \mu d x\right]^{2}}$,

where

$$
x_{r}^{2}=x^{2}+x^{2}-2 x x^{\prime}\left(\mu \mu^{\prime}+\sqrt{1-\mu^{2}} \sqrt{1-\mu^{\prime 2}}\right) \cos \phi
$$

and

$$
v_{0}=3.10 \times 10^{7} \sqrt{E}
$$

It was desired that $\overline{\sigma V} \mathrm{DD}$ and $\overline{\sigma^{V}} \mathrm{DT}$ be each calculated for eleven different energies: $1,2,4,8,16,32,65,125,250,500$, and $1000 \mathrm{kev}$. This was done by use of 22 different $a-v s-x_{r}^{2}$ tables. The $a$ 's were derived from cross sections due to J. L. Tuck. $23 *$ Unequal intervals of $\mathrm{x}_{\mathrm{r}}^{2}$ were used to allow simple trapezoidal interpolation, and a fairly elaborate search procedure made the process as efficient as possible.

After Weddle's rule was applied and terms were rnllerter, the essentials of this cumbersome equation could be symbolically written as

$$
\overline{\sigma v}=C \quad S \mu x / N^{2}
$$

where C stood for constants, $N$ was only subject to the $F(\mu, x)$ distribution function, and $S \mu x$ (the quintuple integral) was revealed as the heart of the calculation.

$$
\overline{\sigma v}=\left[\frac{2 \pi \cdot 2 \cdot 2 \cdot\left(\frac{3}{10} \Delta \mu\right)^{2} \cdot\left(\frac{3}{10} \Delta x\right)^{2}\left(\frac{3}{10} \Delta \phi\right)}{\left[2 \pi \cdot 2\left(\frac{3}{10} \Delta \mu\right) \cdot\left(\frac{3}{10} \Delta x\right)\right]^{2}} \cdot \frac{v_{0}}{\sqrt{E}}\right] \cdot\left[\frac{s_{\mu x}}{\sum_{x_{0}}^{\max } \sum_{R}^{\mu_{R}} F_{i j}} \sum_{0}^{2}\right] \text {, }
$$

\footnotetext{
*Reference 23 of Part A
} 
where

$$
S_{\mu x}=\sum_{x_{0}}^{x_{\max }} \sum_{\mu_{0}}^{\mu_{R}} F_{i j} \sum_{x_{o}}^{x_{\max }} \sum_{-\mu_{R}}^{\mu_{R}} F_{i j} \sum_{o}^{\pi} w_{\phi}^{a\left(x_{r}^{2}\right)_{i j \phi}},
$$

and

$$
F_{i j}=w_{j} x_{j}^{2} w_{i} F\left(\mu_{i}, x_{j}\right)
$$

In the code, the $F_{i j}$ 's were calculated first, then a great deal of time was consumed by the calculation of $\dot{S}_{\mu x}$ 's with respect to the 22 different a tables, the finally the $\mathrm{C}$ and $\mathrm{N}^{2}$ factors were computed and used to get the 22 different: $\bar{\sigma} v^{\prime} s$.

As a time preserving measure, the code could handle concurrently several $F(\mu, x)$ grids having equal $\Delta \mu, \Delta x$, \# $\mu$ and $\# x$. In one particular case, $\overline{\sigma v}$ calculations for normal mode and for peaked-in-angle distribution functions at various $T^{\prime \prime}$ s were performed simultaneously. Computing time was saved because $\sum_{0}^{\pi} w_{\phi} \cdot a\left(x_{r}^{2}\right)$ did not need to be recalculated for each $F(\mu, x)$ grid.

As a check for hidden machine errors, the code printed the partial sums:

$$
S_{\mu x_{\text {partial }}}=\sum_{x_{0}}^{x_{i}} \sum_{\mu_{0}}^{\mu_{i j}} F_{i j} \sum_{x_{o}}^{x_{\max }} \sum_{-\mu_{R}}^{+\mu_{R}} F_{i j} \sum_{0}^{\pi} w_{\psi} a\left(x_{i}^{2}\right) \text {. }
$$

The fusion reaction rate calculation was straightforward and simple. The only coding problems involved were the method of a selection and the minimizing of calculation time. 


\section{APPENDIX B-1}

ELLIPTIC INTEGRAL IN $G(\mu, x)$ EQUATION

$$
\begin{aligned}
& \int_{0}^{2 \pi} v_{r} d \phi=\int_{0}^{2 \pi}\left[v^{2}+v^{\prime 2}-2 v v^{\prime}\left(\mu \mu^{\prime}+\sqrt{1-\mu^{2}} \sqrt{1-\mu^{\prime 2}} \cos \phi\right]^{1 / 2} d \phi\right. \\
& =\int_{0}^{2 \pi}\left[v^{2}+v^{i^{2}}-2 v v^{\prime} \mu \mu^{\prime}-2 v v^{\prime} \sqrt{1-\mu^{2}} \sqrt{1-\mu^{\prime 2}} \cos \phi\right]^{1 / 2} d \phi \\
& =\int_{0}^{2 \pi}\left[v^{2}+v^{\prime 2}-2 v v^{\prime} \mu \mu^{\prime}+\left(2 v v^{\prime} \sqrt{1-\mu^{2}} \sqrt{1-\mu^{\prime 2}}\right)-\left(2 v v^{\prime} \sqrt{1-\mu^{2}} \sqrt{1-\mu^{\prime 2}}\right)\right. \\
& \left.=2 v v^{1} \sqrt{1-\mu^{2}} \sqrt{1-\mu^{2}} \cos \phi\right]^{1 / 2} d \phi \\
& =\int_{0}^{2 \pi}\left[v^{2}+v^{\prime 2}-2 v v^{\prime}\left(\mu \mu^{\prime}-\sqrt{1-\mu^{2}} \sqrt{1-\mu^{\prime 2}}\right)-2 v v^{\prime} \cdot \sqrt{1-\mu^{2}} \sqrt{1-\mu^{\prime 2}}(1+\operatorname{cox} \phi)\right]^{1 / 2} d \phi \\
& =\left[v^{2}+v^{\prime 2}-2 v v^{\prime}\left(\mu \mu^{\prime} \sqrt{1-\mu^{2}} \sqrt{1-\mu^{\prime}}\right)\right]^{1 / 2} \int_{0}^{2 \pi}\left[1-\frac{2 v v^{\prime} \sqrt{1-\mu^{2}} \sqrt{1-\mu^{\prime 2}}(1+\cos \phi)}{v^{2}+v^{\prime^{2}}-2 v v\left(\mu \mu^{\prime}-\sqrt{1-\mu^{2} \sqrt{1-\mu^{\prime}}}\right)}\right]^{1 / 2} d \phi
\end{aligned}
$$

Let

and

$$
q=v^{2}+v^{2}-2 v v^{\prime}\left(\mu \mu-\sqrt{1-\mu^{2}} \sqrt{1-\mu^{i^{2}}}\right)
$$

$$
p=4 v v^{\prime} \sqrt{1-\mu^{2}} \sqrt{1-\mu^{\prime 2}}
$$

Then

$$
\int_{0}^{2 \pi} v_{r} d \phi=q^{1 / 2} \int_{0}^{2 \pi}\left[1-\frac{p}{2 q}(1+\cos \phi)\right]^{1 / 2} d \phi .
$$

Since

$$
1+\cos \phi=2 \cos ^{2} 1 / 2 \phi \text {, let } \beta=1 / 2 \phi, \text { then } 1+\cos \phi=2 \cos ^{2} \beta \text { and } d \phi=2 d \beta,
$$

where $0 \leqslant \phi \leqslant 2 \pi$ and $0 \leqslant \beta \leqslant \pi$.

This can be written

$$
\int_{0}^{2 \pi} v_{r} d \phi=2 q^{1 / 2} \int_{0}^{\pi}\left[1-\frac{p}{q} \cos ^{2} \beta\right]^{1 / 2} d \beta
$$


Let $\gamma=\frac{\pi}{2}-\beta$. Then $\cos \beta=\cos \left(\frac{\pi}{2}-\gamma\right)=\sin \gamma$, and $d \beta=-d \gamma$, where $0 \leqslant \beta \leqslant \pi$, and $\frac{\pi}{2} \geqslant \gamma \geqslant-\frac{\pi}{2}$.

The equation now becomes:

$$
\int_{0}^{2 \pi} v_{r} d \phi=-2 q^{1 / 2} \int_{\frac{\pi}{2}}^{-\frac{\pi}{2}}\left[1-\frac{p}{q} \sin ^{2} \gamma\right]^{1 / 2} \cdot d \gamma=2 q^{1 / 2} \int_{-\frac{\pi}{2}}^{\frac{\pi}{2}}\left[1-\frac{p}{q} \sin ^{2} \gamma\right]^{1 / 2} d \gamma
$$

Since $\sin y$ is squared, the integral is symmetric about $y=0$.

$$
\begin{gathered}
\int_{0}^{2 \pi} v_{r} d \phi=4 q^{1 / 2} \cdot \int_{0}^{\frac{\pi}{2}}\left[1-\frac{p}{q} \sin ^{2} \gamma\right]^{1 / 2} d \gamma \\
\int_{0}^{\frac{\pi}{2}}\left[1-\frac{p}{q} \sin ^{2} \gamma\right]^{1 / 2} d \gamma \text { is a complete elliptic integral of the second kind }
\end{gathered}
$$

so that the integral over $\phi$ can be written:

$$
\int_{0}^{2 \pi} v_{r} d \phi=4 q^{1 / 2} E(k)
$$

where

$$
k=\sqrt{p / q}
$$


This report was prepared as an account of Government sponsored work. Neither the United States, nor the Commission, nor any person acting on behalf of the Commission:

A. Makes any warranty or representation, expressed or implied, with respect to the accuracy, completeness, or usefulness of the information contained in this report, or that the use of any information, apparatus, method, or process disclosed in this report may not infringe privately owned rights; or

B. Assumes any liabilities with respect to the use of, or for damages resulting from the use of any information, apparatus, method, or process disclosed in this report.

As used in the above, "person acting on behalf of the Commission" includes any employee or contractor of the Commission, or employee of such contractor, to the extent that such employee or contractor of the Commission, or employee of such contractor prepares, disseminates, or provides access to, any information pursuant to his employment or contract with the Commission, or his employment with such contractor. 